\title{
DURER ISKANDERANI TEMPOROMANDIBULAR JOINT IMAGING USING CONE-BEAM COMPUTED TOMOGRAPHY
}
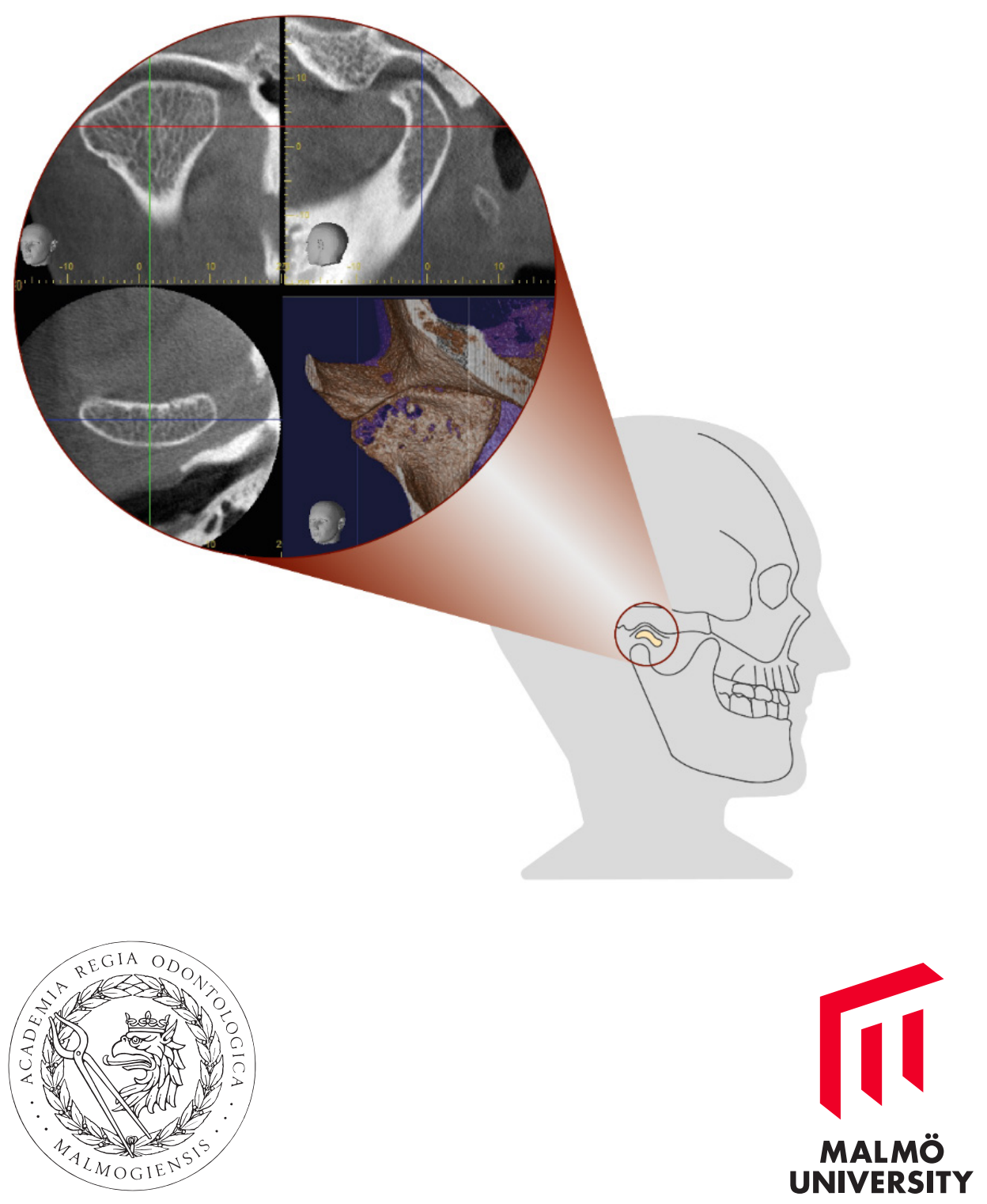

TEMPOROMANDIBULAR JOINT IMAGING USING CONE-BEAM COMPUTED TOMOGRAPHY 


\section{Malmö University, Faculty of Odontology}

Doctoral Dissertation 2020 


\section{DURER ISKANDERANI TEMPOROMANDIBULAR JOINT IMAGING USING CONE-BEAM COMPUTED TOMOGRAPHY}

Department of Oral and Maxillofacial Radiology Faculty of Odontology Malmö University Malmö, Sweden 2020 
This publication is also available in electronic format at:

www.mau.diva-portal.org 
To My Beloved Family, Hussien, Maya \& Malak and My Parents, Faisal \& Hala 



\section{CONTENTS}

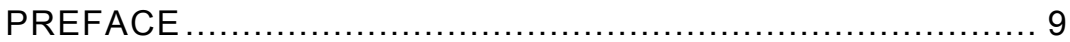

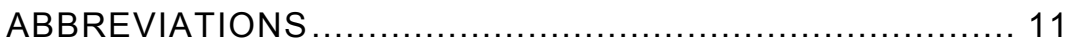

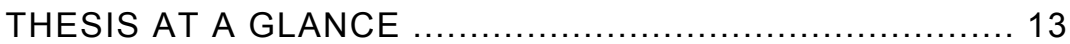

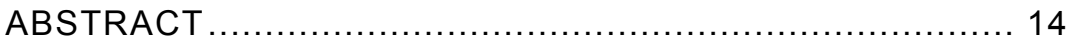

POPULÄRVETENSKAPLIG SAMMANFATTNING $\ldots \ldots \ldots \ldots \ldots 17$

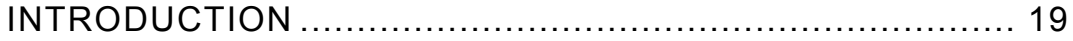

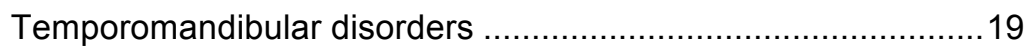

Cone-beam computed tomography .................................23

Image interpretation .............................................. 23

Radiation dosimetry ..............................................25

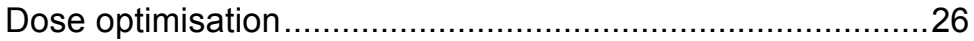

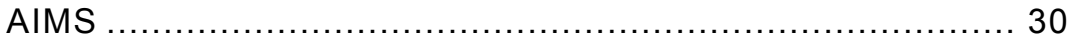

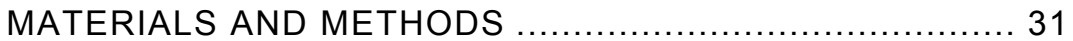

Imaging modalities .............................................................31

Panoramic examinations ............................................... 31

Cone-beam computed tomography examinations ................31

Study I and II ............................................................... 32

Radiographic examinations ........................................ 32

Participated observers.................................................. 33

Radiographic image assessment ..................................33

CBCT educational tools development .................................33

Subjective evaluation of the web-based programme ............34

Data analysis......................................................... 34

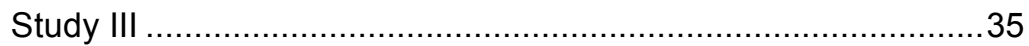

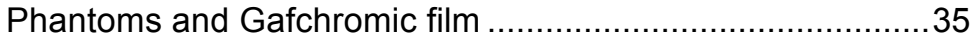

Dose measurements ................................................. 35

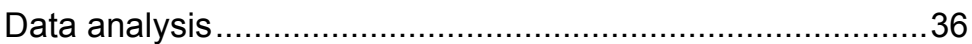




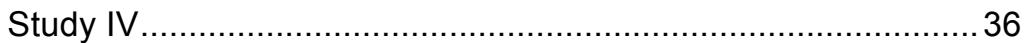

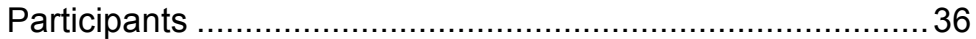

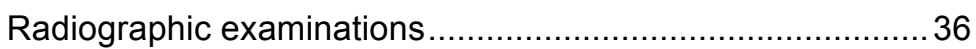

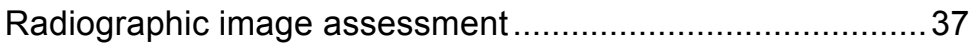

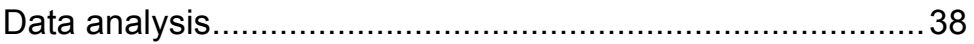

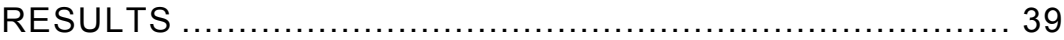

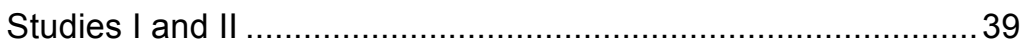

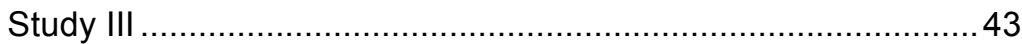

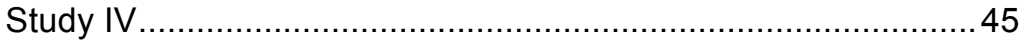

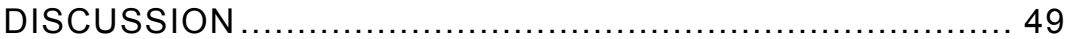

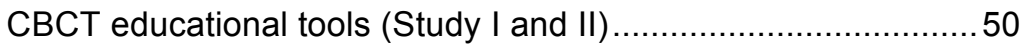

Dose measurements (Study III) ...............................................52

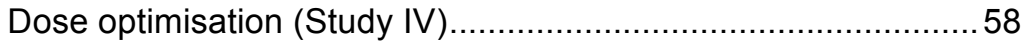

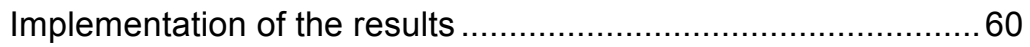

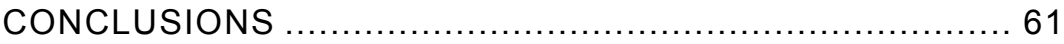

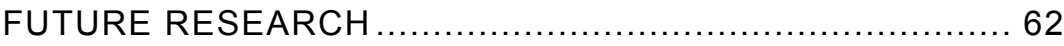

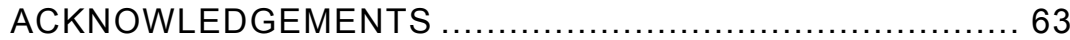

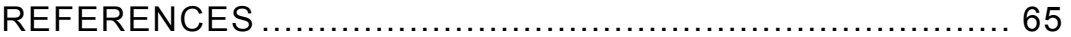

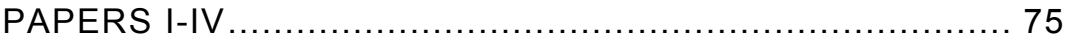




\section{PREFACE}

This thesis is based on the following Papers, which are referred to in the text by their Roman numerals. All papers are reprinted with permission from the copyright holders. The papers are appended at the end of the thesis.

I. Assessment of a training programme on detection of temporomandibular joint osseous changes applying pre-defined 2D multiplane cone-beam computed tomography reconstructions.

Iskanderani D, Alstergren P, Hellén-Halme K.

J Oral Rehabil. 2018; 45:282-288.

II. Web-based educational programme for temporomandibular joint assessment with cone-beam computed tomography.

Iskanderani D, Alstergren P, Ekberg EC, Shi XQ, Hellén-Halme K. J Oral Rehabil. 2020; 00:1-7.

III. Dose distributions in adult and child head phantoms for panoramic imaging and cone beam computed tomography imaging of the temporomandibular joint.

Iskanderani D, Nilsson M, Alstergren P, Hellén-Halme K.

Oral Surg Oral Med Oral Pathol Oral Radiol. 2020; 130:200-208.

IV. Evaluation of a low-dose protocol for cone beam computed tomography of the temporomandibular joint.

Iskanderani D, Nilsson M, Alstergren P, Xie-Qi Shi, Hellén-Halme K. Dentomaxillofac Radiol. 2020; 49:20190495.

The author has performed most of the planning, data collection, data analysis, and writing of the papers in collaboration with the supervisors. All radiographic images are reprinted with patients' permission. 


\section{ABBREVIATIONS}

3D

2D

ALADA

ALARA

$\mathrm{Az}$

CBCT

CT

DC/TMD

DJD

FOV

GDP

Gy

ICRP

$\mathrm{k}$

$\mathrm{kV}$

LD

$\mathrm{mA}$

$\mathrm{mAs}$

MPR

MRI

$\mu \mathrm{Gy}$

NCRP

OSL

PPT

RDC/TMD
Three-dimensional

Two-dimensional

As low as diagnostically acceptable As low as reasonably achievable Area under the ROC curve

Cone-beam computed tomography Computed tomography

Diagnostic Criteria for Temporomandibular Disorder

Degenerative joint disease

Field of view

General dental practitioner

Gray

International Commission on

Radiological Protection

Kappa value

Kilovolt

Luminescent dosimeter

Milliampere

Milliampere second

Multiplanar reconstruction or reformation

Magnetic resonance imaging

Microgray

National Council on Radiation

Protection and Measurements

Optically stimulated luminescence

PowerPoint

Research Diagnostic Criteria for Temporomandibular Disorder 
ROC Receiver operating characteristic

$\mathrm{S}$

Second

SE

Standard error

SEDENTEXCT

Safety and Efficacy of a New and

Emerging Dental X-ray modality

TLD

Thermoluminescent dosimeter

TMJ

Temporomandibular joint

TMD

Temporomandibular disorder 


\section{THESIS AT A GLANCE}

\section{Study I: Observational study}

An educational tool for the assessment of temporomandibular joint osseous tissues applying predefined 2D multiplane cone-beam computed tomography reconstructions was assessed.

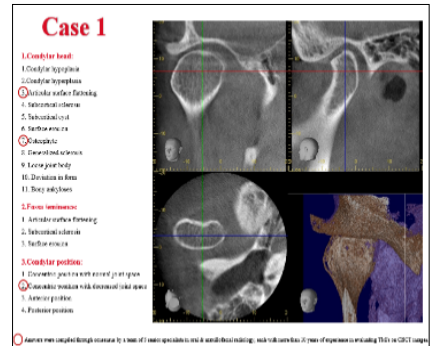

\section{Study II: Observational study}

A web-based programme for temporomandibular joint evaluation using cone-beam computed tomography was developed and evaluated.

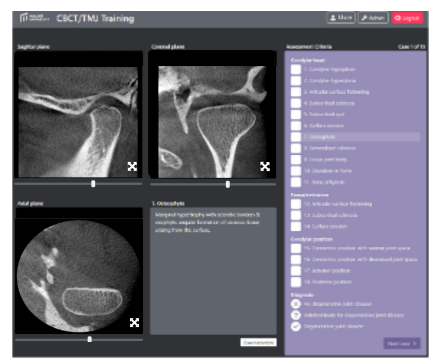

\section{Study III: Phantom study}

Dose distributions were obtained in adult and child head phantoms for panoramic imaging and cone-beam computed tomographic imaging of

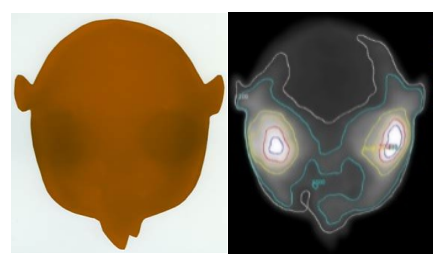
the temporomandibular joint.

\section{Study IV: Clinical study}

A low-dose protocol for cone-beam computed tomography imaging of the temporomandibular joint was

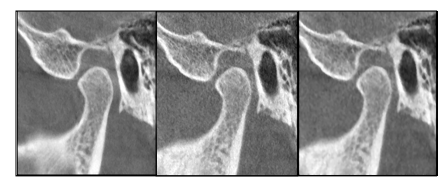
evaluated. 


\section{ABSTRACT}

Cone-beam computed tomography (CBCT) is one of the most revolutionary innovations in dentistry, and was introduced into the dental field two decades ago as a three-dimensional (3D) imaging modality. Since then, it has gained general clinical acceptance, and is widespread among many dental specialties. The CBCT examination is a fast and user-friendly technique that provides multiplanar images with high spatial resolution, providing information that is unattainable with two-dimensional (2D) imaging in many diagnostic tasks. Thus, CBCT is an essential examination tool that can replace or complement other examinations. CBCT imaging plays a valuable role when hard tissue abnormalities are suspected in the temporomandibular joint (TMJ) by providing diagnostic information on cortical and subcortical bone integrity or destruction/production changes.

The growing availability and use of $\mathrm{CBCT}$ at dental clinics has led to concerns as to whether the information obtained by $\mathrm{CBCT}$ imaging justifies the additional exposure of the patient to radiation and the training required to examine and interpret the images. The radiation protection principles: justification and optimisation, should always be applied as the basis for protection. This thesis presents four studies on TMJ imaging using CBCT. In line with recent trends towards digital online education, two CBCT educational tools were developed for the interpretation of CBCT images of the TMJ and published on the Malmö University web site. The first was an educational tool including 35 CBCT examinations of TMJ, presented as 2D multiplane CBCT images. The second was a web-based programme containing 15 CBCT examinations of TMJ, presented as 3D multiplane CBCT images. Both tools included the image analysis criteria of the Diagnostic Criteria for Temporomandibular disorder (DC/TMD) as an assessment module, and were tested by dental students. It was found that they could be useful educational tools for TMJ assessment using CBCT images. 
The aim of the third study was to map and compare the distribution of absorbed doses using radiochromic film dosimeters in panoramic radiography and $\mathrm{CBCT}$ examinations of the TMJ using adult and child anthropomorphic head phantoms. Sheets of Gafchromic film (XR-QA2) were placed at five levels, corresponding to the radiographic examination, in the phantoms. The clinical protocols for panoramic and CBCT imaging of the TMJ of three dental X-ray units were used. The mean absorbed doses to a number of radiosensitive tissues within the oral and maxillofacial regions were estimated. The absorbed doses varied considerably among and within the radiosensitive tissues with examination type, X-ray unit, clinical setting, and patient age. The bone surface and salivary glands received the highest absorbed doses in both radiographic examinations. The radiation burden was lower when using two small right and left fields of view (FOVs) than when using a single larger FOV. Accurate measurements of the absorbed dose in small dental radiation fields is challenging due to steep dose gradients. The use of Gafchromic film has shown promising results, allowing dose comparisons between different radiographic imaging.

The last study was carried out to investigate the possibility of dose optimisation in CBCT examinations of the TMJ, in line with the recommendations of the International Commission on Radiological Protection (ICRP) and the National Council on Radiation Protection and Measurements (NCRP). Thirty-four adult patients referred for CBCT imaging of the TMJ underwent two examinations with different scanning protocols, a manufacturer-recommended protocol (default), and a low-dose protocol in which the tube current was reduced to $20 \%$ of that in the default protocol. Three image stacks were reconstructed: the default protocol, the low-dose protocol, and the low-dose protocol processed using a noise reduction algorithm. Four radiologists evaluated the visibility of TMJ anatomic structures, image quality and radiographic findings. It was found that the visibility of the TMJ anatomical structures, the overall image quality and the radiographic findings using the low-dose protocol were comparable to those in the default protocol, indicating that diagnostically comparable results could be obtained with a five times lower radiation dose. 
Finally, this thesis highlights the diagnostics of TMJ imaging using $\mathrm{CBCT}$ in the context of image interpretation, imaging dosimetry and dose optimisation, all with the purpose of improving and optimising radiological TMJ diagnostics. 


\section{POPULÄRVETENSKAPLIG SAMMANFATTNING}

Denna avhandling belyser olika aspekter vid avbildning med Conebeam computed tomography (CBCT) teknik, allt i syfte att förbättra och optimera käkledsdiagnostiken.

CBCT är en tredimensionell avbildningsmetod som introducerades för cirka två decennier sedan inom tandvården. CBCT-undersökningen är en snabb och användarvänlig teknik som $i$ tre olika plan ger röntgenbilder med hög detaljupplösning. Detta kan medföra att information som inte kan avbildas i vanliga intraorala röntgenbilder kan visas och analyseras. Ett exempel är röntgenundersökningar av käklederna. Dessa kan med fördel avbildas med CBCT teknik när man misstänker hårdvävnadsförändring $\mathrm{i}$ leden Den stora nackdelen med CBCT tekniken är dock att den ger en betydligt högre stråldos till patienterna. Genom strikta kriterier för när $\mathrm{CBCT}$ ska användas och genom noggrann optimering av hur man ska utföra själva undersökningen kan man genomföra undersökningarna med minsta möjliga stråldos men som ändå ger tillräckligt bra bildunderlag för en analys. I Sverige får ansvaret för användning av denna metod enbart innehas av tandläkare med specialistkompetens inom odontologisk radiologi.

Denna avhandling presenterar fyra studier om CBCT och avbildning av käkleden. I linje med den senaste trenden mot digital online-utbildning utvecklades två pedagogiska verktyg för tolkning av CBCT-bilder av käkleder. Den första, ett utbildningsverktyg med 35 CBCTundersökningar, presenterade som 2D-multiplanara CBCT-bilder. Den andra, ett webbaserat program som innehöll 15 CBCT-undersökningar, presenterade som 3D-multiplanara CBCT-bilder. Båda visade sig vara användbara pedagogiska verktyg för träning $\mathrm{i}$ hur hårdvävnadsförändring kan påvisas i CBCT-bilder. 
Syftet med den tredje studien var att kartlägga och jämföra fördelningen av stråldos med hjälp av strålningskänslig film som placerades i två olika fantom, ett som symboliserade en vuxen patient och ett som motsvarade ett barn. Kliniska protokoll för panorama- och CBCTavbildning av käkleden för tre olika röntgenapparater användes. Stråldoserna varierade avsevärt mellan och inom de strålkänsliga vävnaderna med undersökningstyp, röntgenenhet, klinisk inställning och patientens ålder. Benytor samt spottkörtlarna i området erhöll de högsta stråldoserna för båda röntgenundersökningarna.

Den sista studien genomfördes för att undersöka möjligheten till dosopimering vid CBCT-undersökningar av käkleder i linje med rekommendationerna från International Commission on Radiological Protection (ICRP) och National Council on Radiation Protection and Measurements (NCRP). Trettiofyra vuxna patienter genomgick två undersökningar med olika undersökningsprotokoll, ett standardprotokoll och ett lågdosprotokoll där rörströmmen reducerades till $20 \%$. Det visade sig att avbildningen av de anatomiska strukturerna i käkleden, den totala bildkvaliteten och de röntgenfynd som gjordes med lågdosprotokollet var jämförbara med dem för standardprotokollet, vilket indikerar att diagnostiskt jämförbara resultat kunde uppnås med en femtedel av stråldosen. 


\section{INTRODUCTION}

\section{Temporomandibular disorders}

Temporomandibular disorders (TMDs) are a heterogeneous and often multifactorial group of disorders affecting the temporomandibular joint (TMJ), the masticatory muscles, and their associated structures (Figure 1). They represent a major source of orofacial pain of non-dental origin. The TMDs prevalence varied widely due to variation in the studied population, examination methods, and diagnostic criteria, however, they affect up to $12 \%$ of the population, with increased prevalence among younger individuals and women (National Institute of Dental and Craniofacial Research). These disorders commonly have characteristic clinical features such as pain, limited jaw function and joint sound, which can influence daily activities, psychosocial functioning and quality of life. The central attribute of all these disorders is pain, particularly when it becomes chronic, and usually leads to treatment seeking. Other symptoms include headache, jaw asymmetry or locking, neck pain, oral parafunctions and muscle tenderness. The TMDs include pain-related and joint-related disorders, with degenerative joint disease (DJD) being among the most common pathology affecting the TMJ (Tanaka et al., 2008; Ohrbach and Dworkin, 2016). 

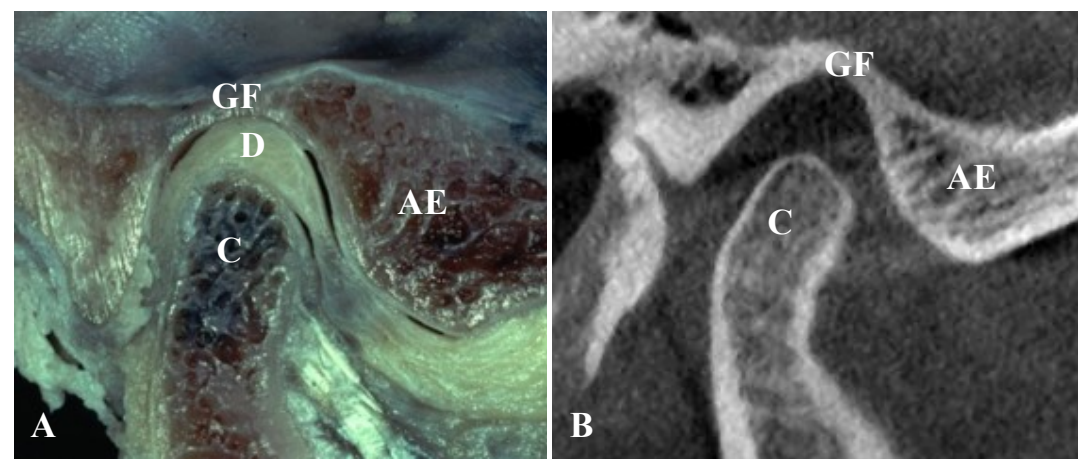

Figure 1. The temporomandibular joint. (A) Photograph of an anatomical dissection (reprinted with permission from Lars Eriksson and Per-Lennart Westesson), and (B) A radiographic image. AE: Articular Eminence, C: Condyle, D: Disc, GF: Glenoid Fossa.

DJD is a non-inflammatory degenerative disease of the joints characterized by destruction and repair of the articular fibrocartilage and underlying osseous structures. The degenerative changes associated with DJD are believed to result from dysfunction of the remodelling capacity of the articular structures due to reduced adaptive capacity or excessive functional loading that exceeds the adaptive capacity (Tanaka et al., 2008). It is commonly related to trauma, the aging process, systemic illness, or hormonal or mechanical factors. A reduction or loss of adaptive response can lead to loss of the articulating cartilage together with gradual progressive destruction of the osseous tissues. Clinically, this can be associated with mild or symptom-free disease, and radiographic examination usually is part of the diagnostic process (Tanaka et al., 2008).

Historically, considerable effort has been devoted to defining a reliable modus for the diagnosis of TMDs (Dworkin and LeResche, 1992). However, many controversies have arisen, together with a lack of consensus among clinicians and researchers, who have reported a lack of scientific evidence supporting aetiology, diagnosis and treatment strategies related to TMDs (Ohrbach and Dworkin, 2016). The introduction of the Diagnostic Criteria for Temporomandibular Disorder (DC/TMD) (Schiffman et al., 2014) formerly RDC/TMD, the Research 
Diagnostic Criteria for TMD (Dworkin and LeResche, 1992) has facilitated TMD diagnostics. It was developed based on a multicentre, population, and experimental and clinical studies. The DC/TMD offers an evidence-based diagnostic system when identifying TMDs, intended to expand scientific knowledge and standardize patient care. It has been proposed that the DC/TMD be used in general dentistry for screening and examination to identify patients suspected of having a TMD, and in decision-making regarding treatment or referral to a specialist, as it would be a part of a clinical examination.

The DC/TMD has been proven to be a reliable and valid comprehensive diagnostic tool, which has been supplemented with descriptions of the most common disorders (Schiffman et al., 2014) and expanded to cover more uncommon disorders (Peck et al., 2014) affecting the TMJ. It also provides definitions of the history, examination, and imaging procedures that are needed to render a reliable clinical diagnosis using decision trees. It therefore helps in the systematic investigation of the patient's complaint in order to obtain the best possible diagnostic information, and consequently to provide the best possible treatment. It is widely accepted, and serves as a sound connection between research and the clinical setting worldwide (Schiffman et al., 2014).

Proper disease management entails a comprehensive process of history reviewing and thorough physical investigations. Although imaging with ionizing radiation is important in augmenting the diagnostic process after ruling out an extracapsular source of pain, the indication for TMJ imaging is based on whether the information gained from the imaging will influence the diagnosis and/or treatment plan. (Petersson, 2010). Panoramic imaging is one of the most widely used dental radiographic investigations as an adjunct to clinical investigations. It provides an overview of the dentition, jaw and alveolar bone, and the TMJ and maxillary sinuses. It has the advantages of being readily accessible and easy to conduct, and both the radiation dose and cost are low. Although it enables the detection of gross osseous changes in the TMJ, it cannot be used to evaluate all the surfaces of the TMJ due to superimposition and distortion. Panoramic imaging has been reported to have poor reliability and low sensitivity for the detection of TMJ osseous changes (Ahmad et al., 2009; Kaimal et al., 2018), and several studies have 
questioned its usefulness for TMJ diagnostics (Ahmad et al., 2009; Leon, 2004; Mawani et al., 2005; Schmitter et al., 2006). Computed tomography (CT) has been recommend as the modality of choice for evaluating the whole spectrum of osseous-related changes in the TMJ (Ahmad et al., 2009; Boeddinghaus and Whyte, 2013). Comprehensive TMJ diagnostic image analysis criteria were developed within the RDCTMD framework by Ahmad et al. in 2009, which proved to be reliable for diagnosing osseous and non-osseous conditions of TMJ using CT and magnetic resonance imaging (MRI), respectively (Ahmad et al., 2009). It was found to be useful in increasing the observer agreement, thus advantageous in order to standardize TMDs diagnostics (Hellen-Halme et al., 2012; Iskanderani et al, 2018). However, the limited availability of CT and cost decrease the use of this technology for dental applications. Cone-beam computed tomography (CBCT) has been found to be superior to $\mathrm{CT}$ in imaging the hard tissues in the maxillofacial region, while the radiation dose to the patient is also considerably lower (Hashimoto et al., 2003; Hashimoto et al., 2007). Comparative studies of the accuracy of TMJ diagnosis have revealed that CBCT is comparable to CT (Honda et al., 2006; Zain-Alabdeen and Alsadhan, 2012). CBCT is thus now considered to be a dose- and costeffective alternative to CT for TMJ osseous evaluation (Honda et al., 2006).

The growing availability of $\mathrm{CBCT}$ units at dental clinics, and the relatively low radiation dose associated with it as a three-dimensional (3D) imaging method, have led to an increase in the number of such investigations. However, concern has arisen as to whether the information obtained with $\mathrm{CBCT}$ imaging justifies the additional exposure of the patient to radiation, and the training required for image interpretation (Horner et al., 2009). Therefore, the work presented in this thesis was carried out to investigate different aspects of CBCT imaging of the TMJ, including image interpretation, imaging dosimetry and dose optimisation. 


\section{Cone-beam computed tomography}

$\mathrm{CBCT}$ is an X-ray imaging technique in which a cone-beam of X-rays is rotated around the object, the signal being collected on a twodimensional (2D) detector. It provides a 3D volumetric dataset composed of a series of $2 \mathrm{D}$ projections. These images are then reconstructed and visualized in different planes, such as multiplanar reconstruction or reformation (MPR), including axial, sagittal and coronal images, reconstructed panoramic images, cross-sectional images, as well as $3 \mathrm{D}$ volumetric rendering. These reconstructions are used for specific anatomic regions and diagnostic tasks. CBCT was first introduced in the field of dentistry in the late 1990s by Mozzo et al. (Mozzo et al., 1998) and Arai et al. (Arai et al., 1999).

CBCT represents a radical change in dental imaging as it overcomes the limitations associated with plain radiographic techniques. The information obtained from CBCT imaging has led to improvements in patient care by improving the diagnosis and/or treatment planning. Since its introduction, CBCT technology has developed rapidly. This has led to increased interest in, and clinical acceptance of, $\mathrm{CBCT}$ among many dental specialties and a wide range of oral and maxillofacial applications, ranging from diagnosis to treatment and follow-up. Compared with $\mathrm{CT}$, the scanning time is shorter and the radiation dose relatively lower, and the images have high spatial resolution allowing fine details to be observed (Miracle and Mukherji, 2009). Images can be acquired using multiple fields of view (FOVs) adapted to the size of the region of interest and the particular diagnostic task, ranging from few centimetres to a full head scan. Several new systems also provide a hybrid solution combining $3 \mathrm{D}$ reconstruction $\mathrm{CBCT}$ images and $2 \mathrm{D}$ acquisition as digital panoramic images and cephalograms.

\section{Image interpretation}

Volumetric images obtained in a $\mathrm{CBCT}$ examination provide extensive and detailed tissue information. The observer can scroll through and 
manipulate the entire scanned volume in many viewing planes and ways, in both two and three dimensions. Therefore, thorough knowledge of CBCT anatomy, anatomic variations, tissue abnormalities and pathologies is required for the interpretation of CBCT images. The clinician who requests or performs CBCT imaging is responsible for generating a task-specific images that provide an answer to the clinical question based on examination of the dataset. It is thus very important that clinicians have the skills required for the interpretation of CBCT images. Studies on under- and/or postgraduate dental education have revealed that little is known regarding some basic aspects of CBCT imaging, such as the appearance of normal anatomy and pathology in CBCT images, radiation safety, patient dose, and image interpretation (Lavanya et al., 2016; Buchanan et al., 2017). Further studies and educational materials targeting some of these gaps are thus necessary to increase knowledge and further the educational process.

Various educational tools are available for improving skills in the interpretation of CBCT images (Al-Rawi et al., 2007; Hassan et al., 2007; SEDENTEXCT Project). However, they are limited in number and scope. Recent advances in digital and web technologies, and their use in education, have focused attention on alternative methods of education, and digital online learning is now a common and promising method of providing information. Several studies have highlighted the advantages of using computer-assisted learning in dental education, including the facts that it is useful, practical, cheap, or sometimes free, can easily be updated, and is accessible at any time or place, in contrast to traditional study materials (Francis et al., 2000; Clark, 2001; Scarsbrook et al., 2005; Al-Rawi et al., 2007; Mitov et al., 2010). Furthermore, online learning has been found to have similar outcomes to traditional learning, as well as being well-received by students (Howerton et al., 2004; AlRawi et al., 2007).

\section{Knowledge gap}

A shortage of educational tools and training material for the interpretation of CBCT images of the TMJ motivated the development of tools that could facilitate dental education in the field of radiographic interpretation. 


\section{Radiation dosimetry}

Radiation dosimetry involves the measurement and assessment of the energy imparted to matter by ionizing radiation. The absorbed dose, i.e., the energy deposited per unit mass, is the fundamental physical dose quantity that is related to biological, chemical, or physical changes in the material or in living tissue. The mean absorbed dose to a specified organ or tissue can be referred to as the organ dose (Dance and Castellano, 2014). Thus, measuring the organ dose allows the associated potential harm to be estimated.

The energy imparted to the patient can be determined by measuring the distribution of the absorbed dose (Carlsson et al., 1999). In dental and maxillofacial radiology, this can be measured using anthropomorphic head phantoms made of human-tissue-equivalent materials to simulate the X-ray attenuation and scattering properties of the tissues, thus simulating the clinical situation. Different dosimetric approaches can be used with phantoms such as luminescent dosimeters (LDs) and films. The extent of the field used in dental X-ray examinations is usually small, and characterized by steep dose gradients, which means that it is necessary for a dosimeter to be able to measure high and low doses over small distances. Thus, obtaining the dose distribution from point dosimetric measurements using LDs is a significant challenge. Luminescent dosimetry is also a time-consuming technique, and requires careful handling and a large number of dosimeters to estimate the dose distribution. Therefore, using radiochromic film is advantageous in such cases.

\section{Radiochromic film}

Radiochromic films were first introduced for high-dose measurements in radiotherapy. However, several types of film have been developed for applications in diagnostic radiology where the dose is lower ( $\leq 50 \mathrm{~Gy})$. Gafchromic $^{\mathrm{TM}}$ film is a self-developing radiochromic film that is used to measure the absorbed dose and its distribution. It has a radiosensitive layer containing a crystalline diacetylene monomer that polymerizes and darkens when irradiated; the amount of darkening being related to the absorbed dose (Watanabe et al., 2006). Its composition is nearly tissueequivalent. The film is provided in large sheets that can be cut to the 
desired size and shape, and the handling and reading processes are simple. Gafchromic film has evolved through sequential versions, corresponding to different dynamic ranges, i.e., dose sensitivity, for different purposes (Devic, 2011; Das, 2017). The latest versions of Gafchromic film, such as XR-QA and XR-QA2, are characterized by a low dynamic range, which can be used for diagnostic radiology. Gafchromic film has high spatial resolution with low energy dependence, making it suitable as a dosimeter in dental radiology (Butson et al., 2010; Devic, 2011; Das, 2017). However, it requires calibration due to its non-linear sensitometric response (Watanabe et al., 2006). Previous radiation dosimetry studies have reported the feasibility of Gafchromic film, however, few trials have been performed in dental radiology (Rampado et al., 2014; Kadesjö et al., 2015; Hellen-Halme et al., 2013; Al-Okshi et al., 2013).

\section{Knowledge gap}

The limited number of reports in the literature regarding the use of Gafchromic film as a radiation dosimeter for dental imaging led to investigations on its use for panoramic and CBCT imaging of the TMJ.

\section{Dose optimisation}

$\mathrm{X}$-rays are today widely used in the medical care process with everincreasing applications. At the same time, efforts are constantly being made by the radiological community to reduce the amount of ionizing radiation used, and to protect both patients and radiation workers. As part of these efforts, the International Commission on Radiological Protection (ICRP) has defined three fundamental principles of radiological protection: justification, limitation and optimisation (ICRP, 2007).

The principle of justification means that no radiographic examination should be carried out unless its advantages are greater than the harmful effects. The principle of dose limitation means that the total radiation dose to individuals from regulated sources in planned exposure situations other than patient medical exposure shall not exceed the limits recommended by the ICRP (ICRP, 2007). Finally, optimisation of protection means that all exposures should be kept as low as reasonably 
achievable (the ALARA principle) considering economic and societal factors. The ALADA principle (as low as diagnostically acceptable) was later proposed, to emphasize the importance of dose optimisation taking into account the diagnostic task in medical imaging, i.e., the radiation dose to the patient must be appropriate for the intended medical purpose (Bushberg, NCRP, 2015). Therefore, special attention should be paid to justification and optimisation in any radiographic examination in order to limit the risks associated with radiation. As dose optimisation is a multidisciplinary task, radiologists and radiographers [in collaboration with medical physicists] must be aware of the risks and benefits of radiation associated with $\mathrm{CBCT}$ imaging, and must understand and be able to implement the principles of radiation protection. Further edification and training of referring clinicians may also be important.

The CBCT radiation dose varies substantially depending on which $\mathrm{X}$ ray unit and imaging protocol is used. It is not always necessary to obtain images with the least possible level of noise, as some diagnostic tasks do not require high-resolution images to answer the clinical question. Furthermore, the patient may be exposed to an unnecessarily high dose of radiation. Thus, the radiation dose should be adjusted so as to provide an image quality that is suitable for the diagnostic task in question (Lofthag-Hansen et al., 2011). In current dental practice, the exposure settings recommended by the manufacturer, and giving the best image quality, are commonly used in examination protocols. This practice usually produces good image quality, but may lead to a higher radiation dose than necessary.

Several scanning parameters can affect the radiation dose, such as the tube current $(\mathrm{mA})$, tube voltage $(\mathrm{kV})$, exposure time $(\mathrm{s})$, voxel size, FOV and rotation arc. Understanding the exposure parameters, and their relation to the radiation dose and radiographic image quality, is of the utmost importance when optimising the exposure. Dose optimisation is usually achieved by reducing the tube current, using partial rotations, reducing the number of projections, and using larger voxel sizes or selecting a more appropriate FOV. Reducing the tube voltage is an uncommon approach (Yeung et al., 2019). The most straightforward and simple optimisation approach is to adapt the FOV to the size and 
position of the area of interest, as a larger FOV increases the radiation dose to the patient (Lofthag-Hansen et al., 2011). Another practical approach to dose optimisation is to adjust the tube current to a level that provides the clinician with sufficient information for a given diagnostic task, at the expense of image quality (Sur et al., 2010; Pauwels et al., 2014).

The tube current and exposure time determine the number of X-ray photons emitted, and increasing the tube current thus increases the signal measured by the X-ray detector and decreases image noise. The tube current is linearly proportional to the radiation dose. Thus, reducing the tube current by $50 \%$, while keeping the tube voltage and exposure time constant, reduces the delivered radiation dose by half. However, the image quality will also be reduced, principally due to the increase in quantum noise. The influence of the tube voltage on the radiation dose is complicated as it determines both the quality and quantity of the X-ray photons. Reducing the tube current thus results in less image degradation than reducing the voltage (Pauwels et al., 2014). Several studies have proposed the potential for dose reduction through reducing the tube current (Sur et al., 2010; Neves et al., 2014; Pauwels et al., 2014; Pauwels et al., 2015).

Image noise influences both contrast resolution and spatial resolution and, consequently, the representation of anatomical structures on the image. Despite the presence of noise, the radiologist may still be able to see the detail required in the structures to make a reliable diagnosis. Indeed, this enables the optimisation principle to be applied. It is therefore important that the radiologist is aware of the diagnostic task when assessing the outcome of optimisation. Task-based assessment measures the quality of an examination as the ability of an observer to perform a well-defined task, based on a series of images (Reiser, 2014). Image quality in CBCT examinations has usually been based on subjective analysis, which is in turn based on the visibility of anatomical landmarks. Visual grading is a common and practical method used to assess task-specific anatomical structures and image quality in comparison to a reference image. Deciding whether the visibility of anatomical landmarks in reduced image quality justifies the lower dose, would be answered depending on the diagnostic task. 
Knowledge gap

Published data on dose optimisation in relation to different diagnostic tasks are limited, and there is thus a need for CBCT dose optimisation adapted for different imaging indications. 


\section{AIMS}

The overall aim of the studies presented in this thesis was to investigate the diagnostics of TMJ imaging using CBCT, in the context of image interpretation, imaging dosimetry and dose optimisation, all with the common purpose of improving and optimising radiological TMJ diagnostics.

\section{Specific aims}

The specific aims of the studies were:

- to investigate the usefulness of an educational tool for the assessment of TMJ osseous tissues using CBCT images (Study I);

- to develop and evaluate an educational web-based programme for the interpretation of CBCT images of the TMJ (Study II);

- to map and compare absorbed doses distribution with Gafchromic films in panoramic radiography and CBCT examinations of the TMJ using adult and child phantoms (Study III); and

- to evaluate a low-dose protocol for CBCT examination of the TMJ (Study IV). 


\section{MATERIALS AND METHODS}

\section{Imaging modalities}

All radiographic imaging was performed with X-ray units from J. Morita Corp., Kyoto, Japan.

\section{Panoramic examinations}

The exposures were performed with two panoramic combination X-ray units, namely, Veraviewepocs 3D F40 and Veraview X800 (Study III). The imaging protocols and exposure parameters used for panoramic examinations are given in Table 1.

Cone-beam computed tomography examinations

Three CBCT units were used: Veraviewepocs 3D F40 was used in all the studies, while Veraview X800 and 3D Accuitomo 170 were used in Study III. The imaging protocols and exposure parameters used for CBCT examinations are given in Table 1. 
Table 1. Imaging protocols and exposure parameters for the radiographic imaging used in all studies

\begin{tabular}{|c|c|c|c|c|c|c|}
\hline 胥 & Protocol & X-ray unit & $\begin{array}{c}\text { Tube } \\
\text { voltage } \\
(\mathrm{kV})\end{array}$ & $\begin{array}{c}\text { Tube } \\
\text { current- } \\
\text { exposure } \\
\text { time } \\
\text { product } \\
\text { (mAs) }\end{array}$ & $\begin{array}{c}\text { Rotation } \\
\text { angle } \\
\left({ }^{\circ}\right)\end{array}$ & $\begin{array}{l}\text { FOV } \\
(\mathrm{cm})\end{array}$ \\
\hline I, II & CBCT, TMJ, A & \multirow{3}{*}{$\begin{array}{l}\text { Veraviewepocs } \\
\text { 3D F40 }\end{array}$} & 90 & 47 & 180 & $4 \times 4$ \\
\hline \multirow[t]{18}{*}{ III } & Panoramic, A & & 70 & 74 & 220 & N/A \\
\hline & Panoramic, C & & 64 & 65 & 220 & N/A \\
\hline & Panoramic, A & \multirow{2}{*}{ Veraview X800 } & 75 & 119 & 220 & N/A \\
\hline & Panoramic, C & & 75 & 104 & 220 & N/A \\
\hline & CBCT, TMJ, A & \multirow{2}{*}{$\begin{array}{l}\text { Veraviewepocs } \\
3 \mathrm{D} \mathrm{F} 40\end{array}$} & 90 & 47 & 180 & $4 \times 4$ \\
\hline & СВCT, TMJ, C & & 90 & 47 & 180 & $4 \times 4$ \\
\hline & CBCT, TMJ, A & \multirow{4}{*}{ Veraview X800 } & 100 & 107 & 360 & $4 \times 4$ \\
\hline & CBCT, TMJ, A & & 100 & 56 & 180 & $4 \times 4$ \\
\hline & СВCT, TMJ, C & & 100 & 90 & 360 & $4 \times 4$ \\
\hline & СBCT, TMJ, C & & 100 & 47 & 180 & $4 \times 4$ \\
\hline & CBCT, TMJ, A & \multirow{8}{*}{$\begin{array}{l}\text { 3D Accuitomo } \\
170\end{array}$} & 90 & 105 & 360 & $4 \times 4$ \\
\hline & CBCT, TMJ, A & & 90 & 54 & 180 & $4 \times 4$ \\
\hline & CBCT, TMJ, C & & 90 & 105 & 360 & $4 \times 4$ \\
\hline & CBCT, TMJ, C & & 90 & 54 & 180 & $4 \times 4$ \\
\hline & СBCT, TMJ, A & & 90 & 105 & 360 & $14 \times 5$ \\
\hline & CBCT, TMJ, A & & 90 & 54 & 180 & $14 \times 5$ \\
\hline & CBCT, TMJ, C & & 90 & 105 & 360 & $14 \times 5$ \\
\hline & CBCT, TMJ, C & & 90 & 54 & 180 & $14 \times 5$ \\
\hline \multirow[t]{2}{*}{ IV } & $\begin{array}{l}\text { CBCT, TMJ, A } \\
\text { Default }\end{array}$ & \multirow{2}{*}{$\begin{array}{l}\text { Veraviewepocs } \\
3 \text { D F40 }\end{array}$} & 90 & 47 & 180 & $4 \times 4$ \\
\hline & $\begin{array}{l}\text { CBCT, TMJ, A } \\
\text { Low-dose }\end{array}$ & & 90 & 9.4 & 180 & $4 \times 4$ \\
\hline
\end{tabular}

N/A, not applicable; A, adult; C, child; FOV, field of view

\section{Study I and II}

\section{Radiographic examinations}

Fifty CBCT examinations of TMJ, which were obtained for the assessment of DJD, were retrieved from the database at the Faculty of Odontology, Malmö University for Studies I and II. None of the 
examinations could be related to any other underlying disease or malignancy, and they covered a wide range of osseous changes in the TMJ. Images of TMJs showing no osseous changes were also included. All personal, clinical and radiograph-related data were anonymized. Study I included 35 of these examinations, and 3 MPR images for each examination were exported. The remaining 15 examinations were used in Study II, and 3 MPR volumes for each examination were exported and converted to JPEG file format to be compatible with web browsers using Synedra image viewer.

\section{Participated observers}

Seven International Master's dental students who were general dental practitioners (GDPs), took part as observers in Study I, and 36 undergraduate dental students in their final year at the Faculty of Odontology, Malmö University, Malmö, Sweden, participated in Study II. None of them had any previous training in the interpretation of CBCT images of the TMJ.

\section{Radiographic image assessment}

The assessment module used in Studies I and II for the TMJ osseous tissues was structured according to the DC/TMD image analysis criteria (Ahmad et al., 2009). The reference findings were compiled through consensus by three oral and maxillofacial radiologists.

\section{CBCT educational tools development}

Two PowerPoint (PPT) files (Study I) and a web-based programme (Study II) were developed as educational tools for the assessment of TMJ osseous tissues in CBCT images. Both tools presented the CBCT examinations of the TMJ together with the assessment module. The first PPT file was used as a training session and included five CBCT examinations of the TMJ. The second PPT file included $30 \mathrm{CBCT}$ examinations, which were assessed by the observers who participated in the training session. The observers assessed the images twice without a 
training period in between. (The two PPT files can be accessed at https://mau.app.box.com/s/rs6t84nxxac8ibe3bnzaq9su9b18dle0.

The web-based programme was designed and implemented with the help of professional web designers (programme development described in detail in Paper II). During image assessment, it enables the observer to scroll through the whole image volume and enlarge the images. The observers were given an introduction to the programme, and then assessed the examinations on their own computers, twice [given an open accessibility to the programme in between the two assessments for practice]. Their responses were saved in real-time on a remote server, and the examination cases were presented in randomized order each time the programme was started. The programme is now freely available at http://www.cbct-tmj.mau.se.

\section{Subjective evaluation of the web-based programme}

The participated observers were asked to evaluate their experience of using the web-based programme. The questions concerned accessibility, design, radiographic image quality, the results matrix, the knowledge gained, and the web-based programme as a learning tool. Responses were given on a 5-point Likert scale: 1, extremely satisfied; 2, moderately satisfied: 3, neutral; 4, moderately dissatisfied and 5, extremely dissatisfied.

\section{Data analysis}

The observers' performance with the CBCT educational tools was expressed as the percentage of correct findings. The changes in overall response between the first and second assessments were tested using the pairwise $t$-test in Study I and the sign test in Study II. The level of significance $(p)$ was set to $\leq 0.05$. 


\section{Study III}

\section{Phantoms and Gafchromic film}

Two cross-sectional anthropomorphic head phantoms for absorbed dose measurements were used in Study III: an adult RANDO phantom and a 10-year-old child CIRS phantom (Figure 2A). The sectional surfaces are flat and smooth. The adult phantom is made of human bone and the child phantom is made of bone-equivalent material; both embedded in soft-tissue-equivalent materials. Organs of interest were traced for each selected phantom slices. Gafchromic XR-QA2 films were used for absorbed dose measurements (Figure 2B). These were calibrated against an ionization chamber before use, as described in detail in Paper III.
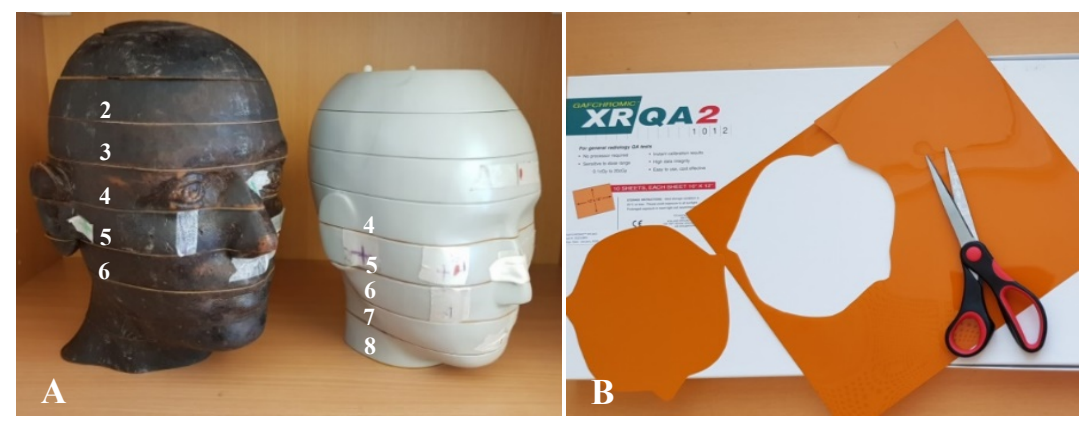

Figure 2. (A) The two anthropomorphic head phantoms loaded with Gafchromic film at five levels, as indicated by the numbers. Left: the adult phantom, for temporomandibular joint examination, and right: the child phantom, for panoramic examination. (B) Gafchromic film cut to the correct size and shape.

\section{Dose measurements}

The phantoms were loaded with Gafchromic films at five levels. For panoramic examinations, the films were centred around the occlusal planes, while for the CBCT examinations, they were centred around the TMJ region. The phantoms were carefully placed so that the central beam of the radiation field did not coincide with the film at the third level, i.e. the film at the third level had a slight offset in the $\mathrm{Z}$ direction 
(length axis of the phantom). Multiple exposures were made to obtain a dose that was within the dynamic range of the film. The exposed films were scanned using an Epson Perfection 4990 Photo flatbed scanner and converted into 8-bit greyscale images using ImageJ software. Net pixel values were calculated (mean pixel value in the exposed image minus the mean pixel value for the background). The absorbed doses were estimated by applying the dose calibration equation to the net pixel value data after converting these data into 32-bit floating-point data.

The absorbed dose matrix was then divided by the number of exposures, which provided an absorbed dose matrix for 1 exposure, as in the clinical situation. The mean doses absorbed by the radiosensitive structures within the oral and maxillofacial region (brain, eyes, salivary glands, oral mucosa and bone surface) were estimated by overlapping the traced organs on the dose distribution matrices and then calculating the mean absorbed dose inside each organ.

\section{Data analysis}

The relative radiation burden and mean absorbed dose to the radiosensitive tissues were compared according to the examination type, $\mathrm{X}$-ray unit, clinical settings and phantom.

\section{Study IV}

\section{Participants}

Thirty-four adult patients (mean age 57 years) who had been referred for CBCT examination of the TMJ were recruited for Study IV. The Regional Ethics Review Board in Lund, Sweden, approved this study (Dnr. 2017/434).

\section{Radiographic examinations}

The participants underwent two consecutive CBCT examinations: one using the default (manufacturer's) protocol and the other with a lowdose protocol. The CBCT examinations were reconstructed in sagittal volumes bilaterally (giving 68 volumes). These were retrieved, 
anonymized, randomly coded and grouped into three groups using the ImageJ software: a default protocol, a low-dose protocol, and a processed protocol, which is low-dose stack processed with a noise reduction algorithm (giving a total number of 204 stacks) (Rudin et al., 1992) (Figure 3).

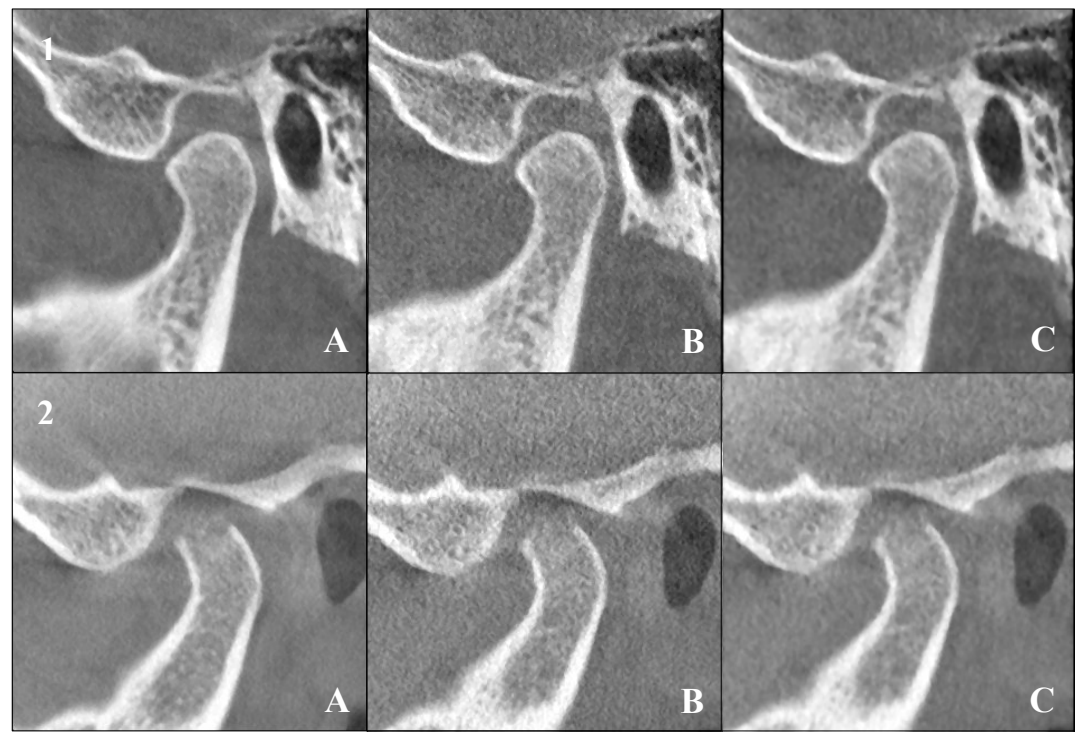

Figure 3. Cone-beam computed tomography images for two temporomandibular joints using the three protocols: (A) default, (B) low-dose, and $(\mathrm{C})$ processed. 1 (A, B, and $\mathrm{C}$ ) show no signs of degenerative joint disease, while $2(\mathrm{~A}, \mathrm{~B}$, and $\mathrm{C})$ show signs of degenerative joint disease.

\section{Radiographic image assessment}

Four calibrated oral and maxillofacial radiologists evaluated all the images. They independently assessed the visibility of five anatomical structures in the TMJ region, namely: the outlines of the condyle, the articular eminence, and the articular fossa, and the trabecular patterns of the condyle and the temporal bone, under the same viewing conditions. A three-point scale was used to assess the visibility of each anatomical 
structure: 1 , definitely visible; 2 , questionably visible and 3 , not visible. Furthermore, the observers were asked to give their overall subjective impression of image quality ranked as: 1 , diagnostically acceptable; 2 , diagnostically questionable and 3 , not diagnostically acceptable. To assess the radiographic findings and thereby, the diagnostic performance of the low-dose protocols, the observers were asked to record their level of confidence concerning the presence of DJD as 1, definitely not; 2 , probably not; 3 , questionable; 4 , probably and 5 , definitely, according to the DC/TMD (Ahmad et al., 2009). Intraobserver agreement was determined by asking each observer to re-evaluate 40 TMJs after an interval of at least 14 days.

\section{Data analysis}

The data from the observed TMJ anatomical structures and overall image quality ratings were pooled and compared pairwise using the sign test. Receiver operating characteristic (ROC) curves were used to analyse the radiographic findings concerning the presence of DJD. The areas under the curves $\left(A_{z}\right)$ were calculated. Intraobserver agreement was estimated using kappa $(k)$ statistics according to Altman (Altman, 1991): $k=0.81-1.00$, excellent; $k=0.61-0.80$, good; $k=0.41-0.60$, moderate; $k=0.20-0.40$, fair; $k<0.20$, poor. 


\section{RESULTS}

\section{Studies I and II}

Two educational tools for the assessment of TMJ osseous tissues in CBCT images were developed, and can be accessed from the Malmö University web site. Both were designed based on DC/TMD image analysis criteria. The first tool consisted of 2 PPT files including predefined 2D multiplane CBCT reconstructions of the TMJ (Figure 4). The second was a web-based programme including 3D multiplane CBCT reconstructions (Figure 5).

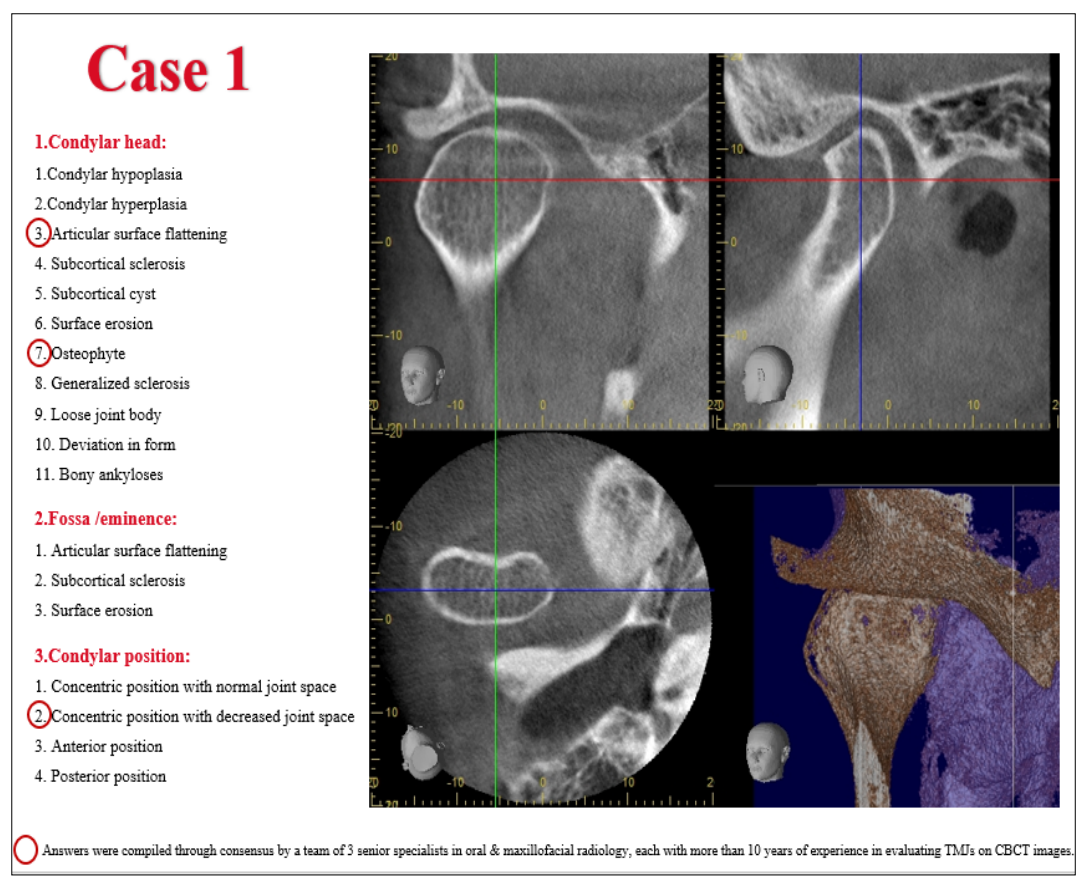

Figure 4. A screenshot of the educational tool that presented in Study I for the assessment of TMJ osseous tissues in 2D pre-defined CBCT reconstructions. 


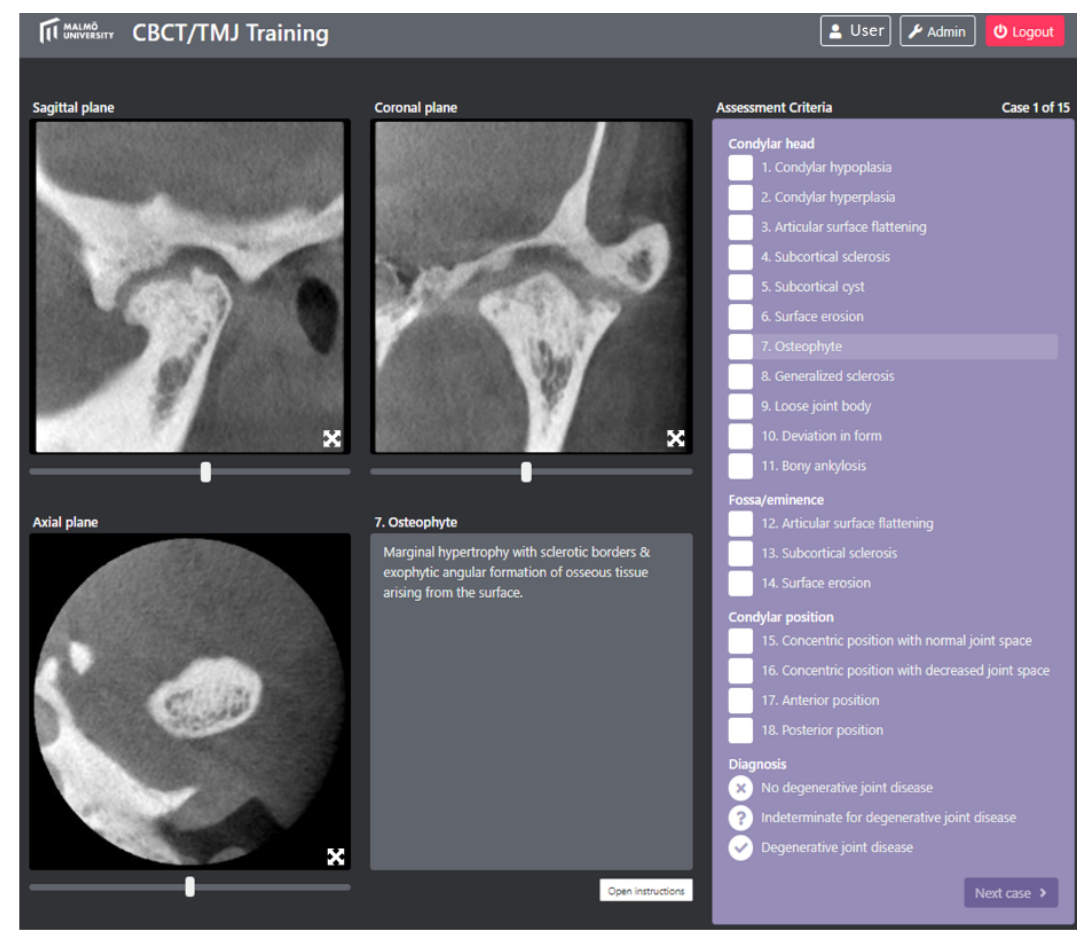

Figure 5. A screenshot of the web-based programme that developed in Study II for the interpretation of CBCT images of the TMJ.

The observers demonstrated good performance in the first assessment in both tools; the mean correct responses being $86 \%$ in Study I and $76 \%$ in Study II. The improvements in the second assessment were not statistically significant $(p>0.05)$, (Figure 6). 


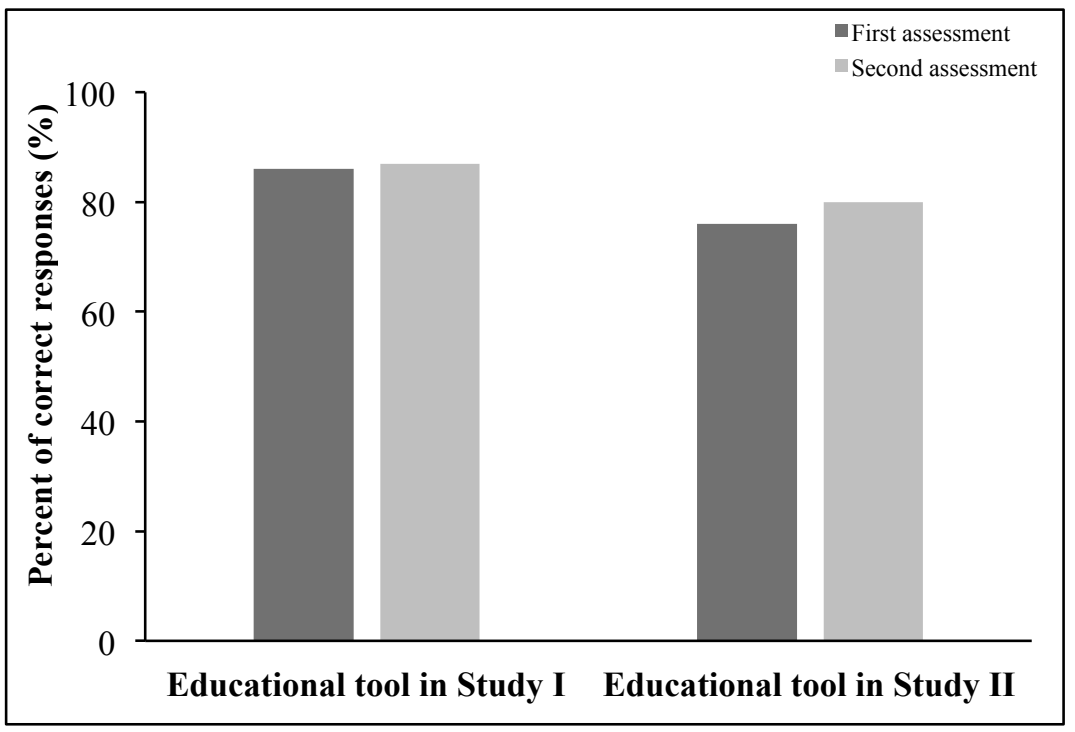

Figure 6. Percentage of correct responses in the two educational tools developed in Studies I and II.

According to the observers' subjective evaluation (Figure 7), more than $90 \%$ were moderately or extremely satisfied with the web-based programme, and thought that it was easy to access and had a userfriendly design, while more than $65 \%$ were satisfied with the radiographic image quality and the results matrix (Figure 8). Seventysix percent of the observers were satisfied with the knowledge gained after using this programme and with its value as a learning tool. 


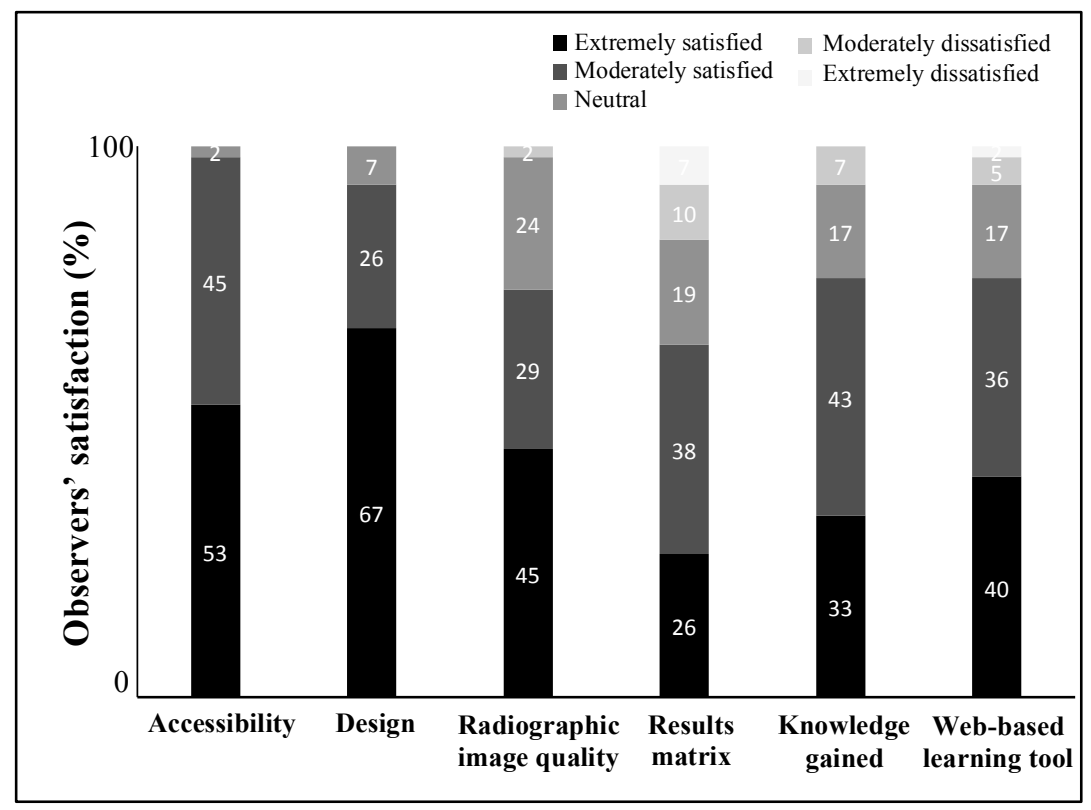

Figure 7. Observers' subjective evaluation of the web-based programme after the second assessment.

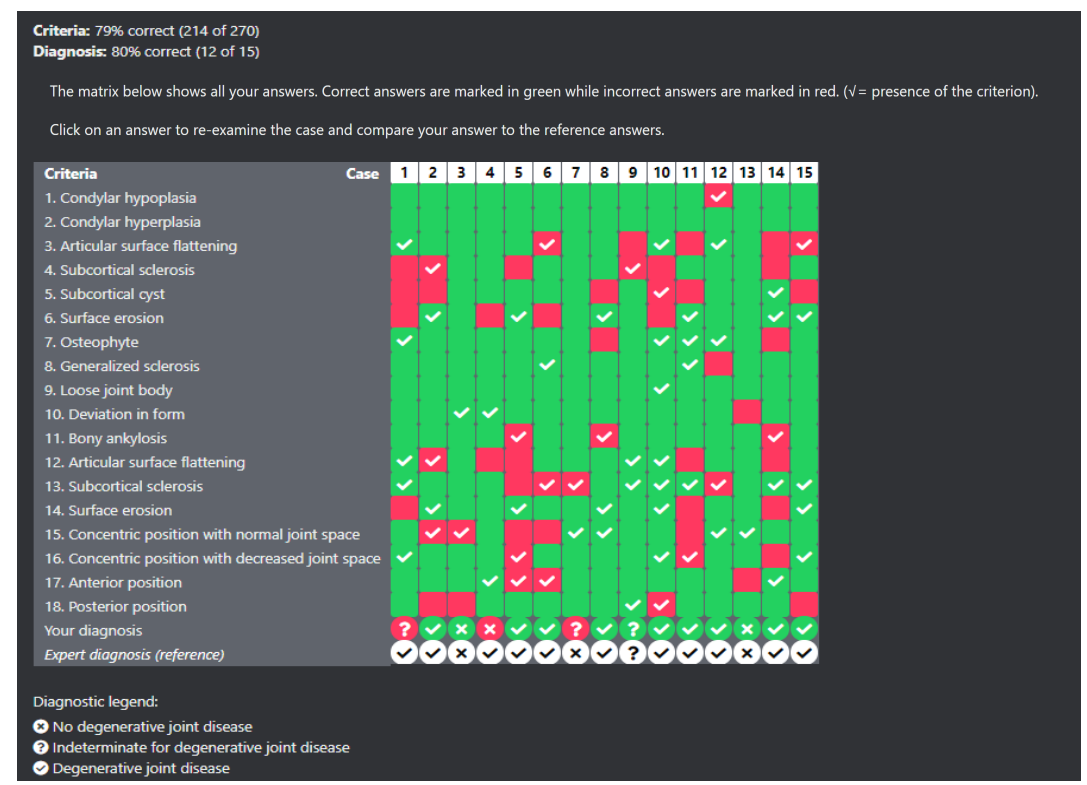

Figure 8. A screenshot of the user's result matrix that compares user and reference responses for all examination cases. 


\section{Study III}

The absorbed doses varied considerably among and within the radiosensitive tissues with all the protocols used, due to variations in the type of examination, the X-ray unit, clinical settings and phantoms. Tables for organ doses in panoramic and CBCT examinations of the TMJ are available in Paper III. Bone surface and salivary glands received the highest absorbed doses in both panoramic and CBCT examinations of the TMJ. The absorbed doses to the other tissues ranged between $70 \mu \mathrm{Gy}$ and $270 \mu \mathrm{Gy}$ in the panoramic examination. In halfrotation CBCT examinations, the absorbed doses varied between 190 $\mu \mathrm{Gy}$ and $760 \mu \mathrm{Gy}$, whereas the corresponding values for full-rotation examinations were $380 \mu \mathrm{Gy}$ and $1530 \mu \mathrm{Gy}$. Absorbed doses distribution in panoramic examination and two CBCT examinations of TMJ with different FOVs illustrated in Figure 9.

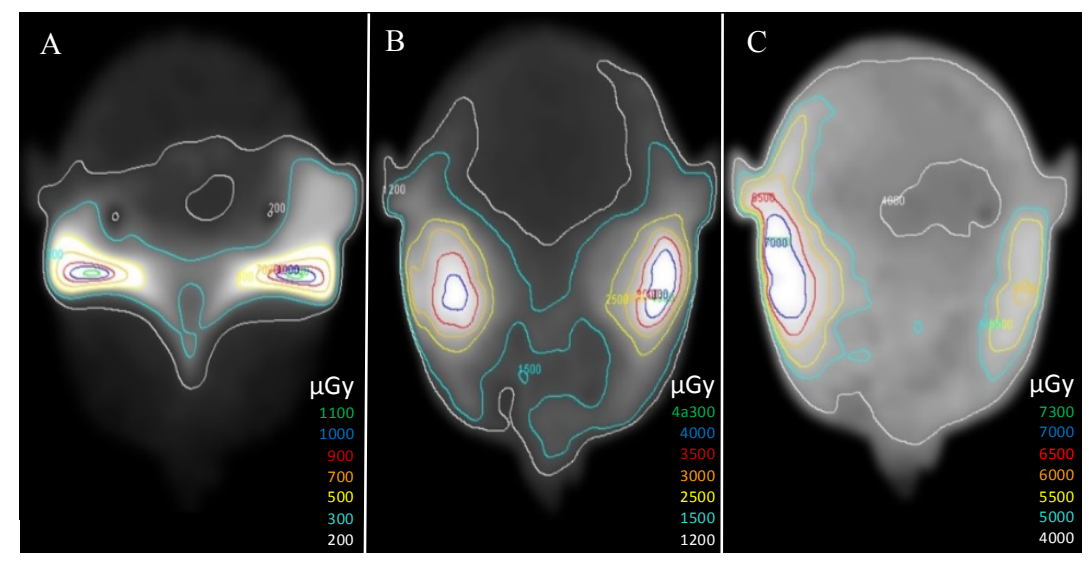

Figure 9. Absorbed doses distribution given as isodose lines in $\mu \mathrm{Gy}$ for the adult head phantom. (A) Panoramic examination, (B) TMJ examination using CBCT with two $4 \mathrm{x} 4 \mathrm{~cm}$ FOVs, (C) TMJ examination using CBCT with one 14 x $5 \mathrm{~cm}$ FOV.

Most of the relative radiation burden to the adult head phantom was slightly higher than that to the child head phantom (Figures 10). Meanwhile, when using two small FOVs, for right and left TMJs examination, the relative radiation burden was lower than when using one large FOV (Figures 11). 


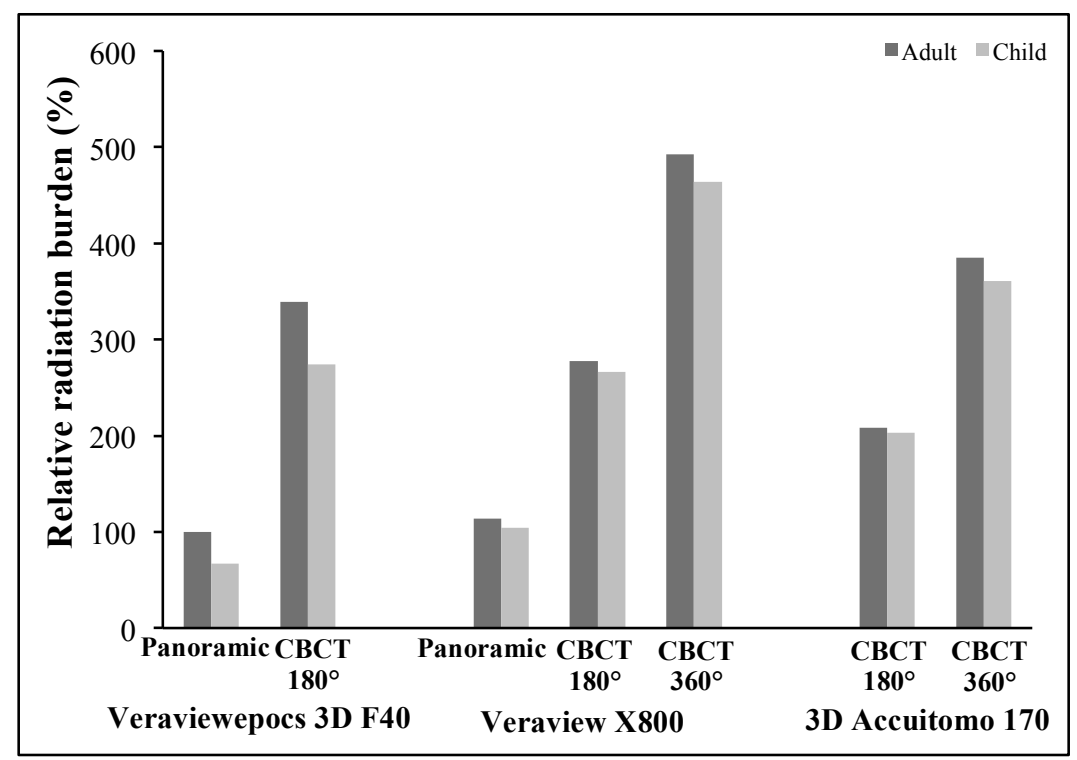

Figure 10. The percentage of relative radiation burden to adult and child head phantoms when comparing different radiographic examinations, rotation angles and $\mathrm{X}$-ray units.

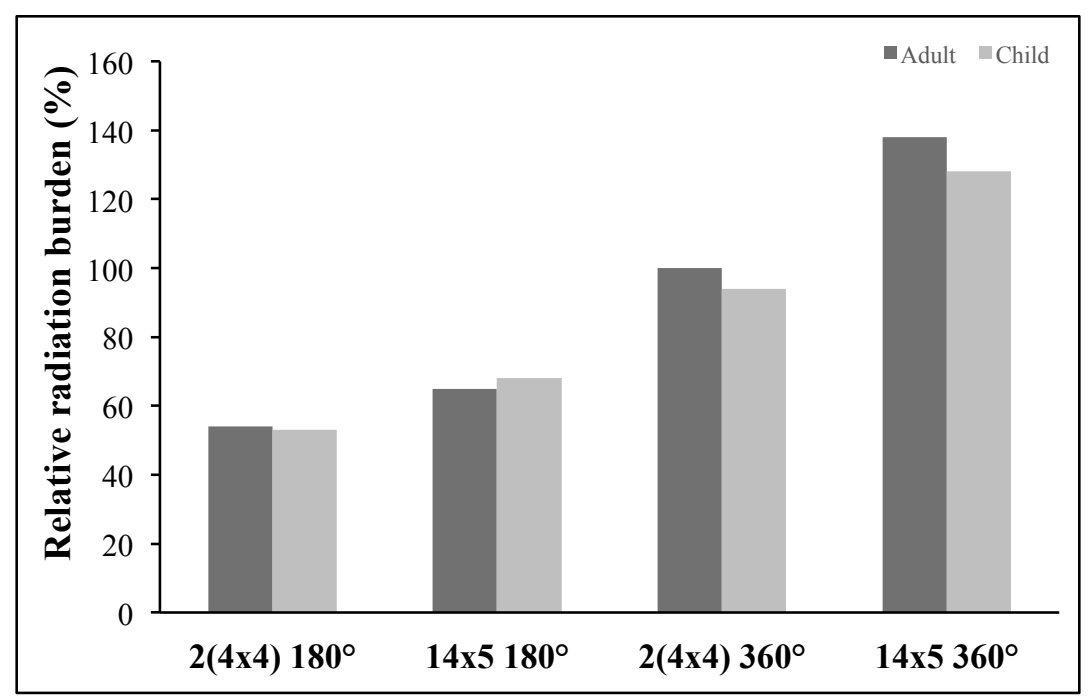

Figure 11. The percentage of relative radiation burden to adult and child head phantoms when comparing different FOVs in CBCT imaging. 
Large differences were found in the radiation burden between the X-ray units used (Figure 10). The Veraviewepocs 3D F40 unit exhibited the highest radiation burden of the three units for half-rotation CBCT scans, while the Veraview X800 unit delivered the highest radiation burden in both panoramic and full-rotation CBCT examinations. The 3D Accuitomo 170 unit delivered the lowest radiation burden during the CBCT examinations, at both full- and half-rotation scans.

\section{Study IV}

Upon establishing the reference standard, two observers evaluated the default protocol using the DC/TMD image analysis criteria (Ahmad et al., 2009). It showed that half of the examinations had radiographic findings indicating the presence of DJD while the rest were without, agreement being obtained among all the examinations except one.

None of the observers reported any difference in the visibility of the five anatomical structures or overall image quality between the default protocol and the two low-dose protocols $(p \leq 0.05)$ (Table 2). Only one observer reported an improvement in the visibility of the structures and image quality between the low-dose protocol and the processed protocol (Observer 3, Table 2).

Table 2. Level of statistical significance $(p)$ for the visibility of five anatomical structures and image quality of CBCT images produced using the three protocols: default protocol, low-dose protocol, and processed protocol

\begin{tabular}{|c|c|c|c|c|c|}
\hline & Protocol & 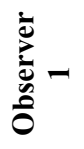 & 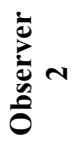 & 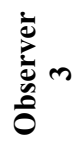 & 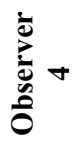 \\
\hline \multirow{3}{*}{ Visibility } & Default vs. Low-dose & 0.00 & 0.00 & 0.00 & 0.00 \\
\hline & Default vs. Processed & 0.00 & 0.00 & 0.00 & 0.00 \\
\hline & Low-dose vs. Processed & 0.00 & 0.00 & 0.79 & 0.00 \\
\hline \multirow{3}{*}{$\begin{array}{l}\text { Image } \\
\text { quality }\end{array}$} & Default vs. Low-dose & 0.00 & 0.00 & 0.04 & 0.00 \\
\hline & Default vs. Processed & 0.00 & 0.00 & 0.04 & 0.00 \\
\hline & Low-dose vs. Processed & 0.00 & 0.00 & 1.00 & 0.00 \\
\hline
\end{tabular}


No statistically significant difference was observed in the radiographic findings concerning the presence of DJD between the default protocol and the two low-dose protocols $(p \leq 0.05)$ (Table 3$)$. The mean area under the ROC curves for all observers was 0.93 for the low-dose protocol and 0.94 for the processed protocol. Figures 12 and 13 show the superimposition of the ROC curves for the low-dose and processed protocols, respectively, assessed by all the observers according to the radiographic findings concerning the presence of DJD.

Table 3. Area under the receiver operating characteristic curves $\left(A_{z}\right)$ when four observers assessed the radiographic findings concerning the presence of DJD in CBCT images produced using the default protocol as reference standard, for the low-dose protocol and the processed protocol

\begin{tabular}{|c|c|c|c|c|c|c|}
\hline \multirow{2}{*}{} & \multicolumn{3}{|c|}{ Low-dose protocol } & \multicolumn{3}{c|}{ Processed protocol } \\
\cline { 2 - 7 } & $\mathbf{A}_{\mathbf{z}}$ & SE & $\boldsymbol{p}$-value & $\mathbf{A}_{\mathbf{z}}$ & $\mathbf{S E}$ & $\boldsymbol{p}$-value \\
\hline Observer 1 & 0.92 & 0.04 & 0.00 & 0.95 & 0.03 & 0.00 \\
\hline Observer 2 & 0.95 & 0.03 & 0.00 & 0.94 & 0.03 & 0.00 \\
\hline Observer 3 & 0.92 & 0.03 & 0.00 & 0.93 & 0.03 & 0.00 \\
\hline Observer 4 & 0.94 & 0.03 & 0.00 & 0.94 & 0.03 & 0.00 \\
\hline
\end{tabular}

$\mathrm{SE}=$ standard error 


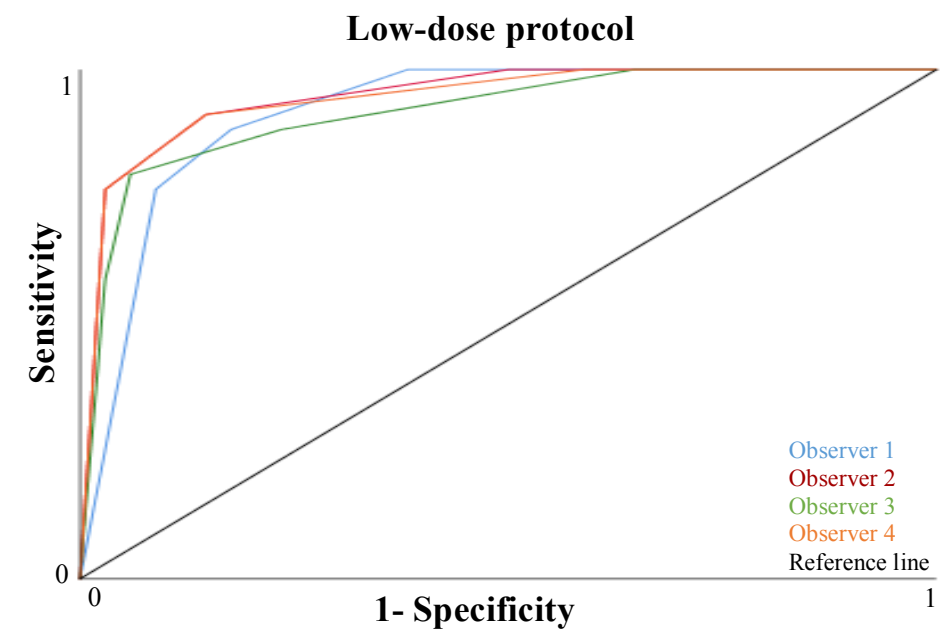

Figure 12. Receiver operating characteristic curves for assessing the radiographic findings concerning the presence of DJD by four observers when using the low-dose protocol.

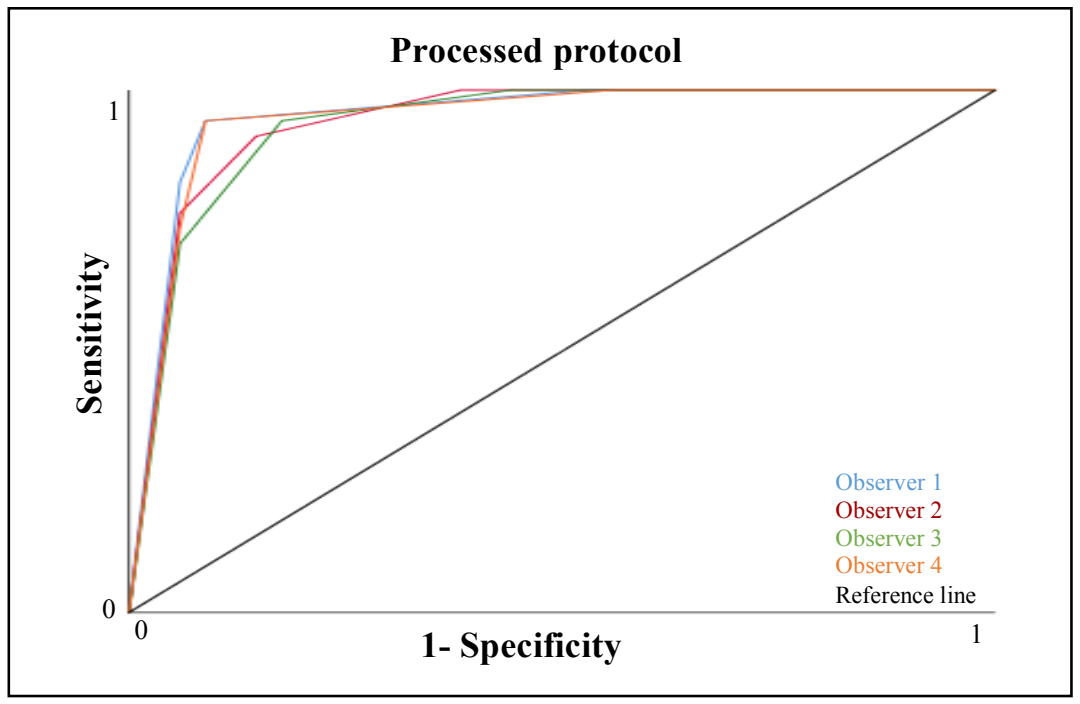

Figure 13. Receiver operating characteristic curves for assessing the radiographic findings concerning the presence of DJD by four observers when using the processed protocol. 
Intraobserver agreement regarding the radiographic findings of the presence of DJD was good to very good for all observers $(k$ values $=$ $0.75-0.85)$. 


\section{DISCUSSION}

Radiographic imaging adds a new dimension to the medical diagnostic procedure by allowing clinicians to see structures inside the body in two or three dimensions, without surgical procedures; the greater goal being to treat patients more efficiently and effectively. It may be used for excluding or confirming suspected disease and/or adding information when the clinical diagnosis is uncertain. The popularity of dental CBCT imaging has been growing since its introduction in the late 1990s. However, there is still controversy concerning different aspects such as the radiation dose, the diagnostic efficacy in different diagnostic tasks, the time needed to interpret the image, the cost, knowledge of 3D anatomical structures reconstructed by $\mathrm{CBCT}$ images, and image interpretation skills. Nevertheless, it plays an important role in the diagnosis and treatment of many dental and osseous diseases in the jaws.

Radiographic imaging should be used to obtain a definitive diagnosis of osseous-related pathology in the TMJ, although referral for TMJ imaging must be justified based on individual assessment. CBCT examination of the TMJ has acceptable diagnostic accuracy for osseous TMJ abnormalities together with high specificity and fairly high sensitivity, and has been reported to be comparable to CT (Honda et al., 2006; Sirin et al., 2010; Zain-Alabdeen and Alsadhan, 2012). The indication for CBCT imaging of the TMJ is related to the clinician's questions after obtaining a detailed medical history and thorough clinical investigation. These questions are usually related to failed treatment, persistent symptoms without explanation, or other questions that could be resolved by a CBCT examination, which thus plays a role in diagnosis and/or treatment planning (Petersson, 2010). In the work described in this thesis, different aspects of CBCT imaging of the TMJ were studied, namely image interpretation, imaging dosimetry, and dose optimisation. The first two studies present two educational tools for the 
interpretation of CBCT images of the TMJ. The dose distribution was assessed using Gafchromic film in Study III, and a low-dose protocol for CBCT imaging of the TMJ was evaluated in Study IV.

\section{CBCT educational tools (Study I and II)}

During the evaluation of the three-dimensional CBCT datasets, a broad and systematic approach is recommended. The observer can reorient, and manipulate the images in many viewing planes. Thus, such images provide extensive and detailed information. The observer needs to assess all the structures in the FOV and gather as much as possible information to answer clinical questions and observe any incidental findings. However, thorough knowledge of 3D anatomy images, anatomic variations, tissue abnormalities, and pathologies is required to interpret $\mathrm{CBCT}$ images, and this is not extensively covered during undergraduate dental education.

The need to strengthen the knowledge of TMJ diagnostics, treatment decisions among GDPs as well as continuous education were addressed (Lindfors et al., 2016). Digital online educational tools could provide a means of increasing knowledge and coherence among the users worldwide. In addition, as is the case with the current global situation where the educational process has largely been transferred to the internet, the development of digital online educational tools should be encouraged to support the education. Well-defined criteria, together with calibrated practitioners, will facilitate image interpretation for the evaluation of TMJ pathology (Petersson, 2010). The reliability and validity of the DC/TMD image analysis criteria have been critically appraised providing a solid basis for TMJ evaluation, which is now widely accepted (Ahmad et al., 2009). The application of these criteria could help in providing standardized knowledge and thus facilitate the diagnostic process. Hellen-Halme et al. (Hellen-Halme et al., 2012) have confirmed the value of these criteria, and suggested that their use could improve the consensus or agreement of the observers, thus increase the diagnostic outcome.

The educational tool presented in Study I was intended to provide dental practitioners an overview for the assessment of osseous tissues in the 
TMJ through a number of CBCT examinations presented as pre-defined 2D multiplane $\mathrm{CBCT}$ reconstructions. The observers were able to identify osseous changes, and their ability to identify TMJs without osseous changes were even better. As a result of these findings, it was decided to expand the project by developing a web-based programme that simulates the clinical situation, allowing the observers to evaluate the whole $\mathrm{CBCT}$ volume and diagnose the DJD case.

During development of the web-based program, CBCT DICOM images were converted to least compressed JPEG format. The conversion was performed to manage the size of images to fit within the web pages, with reasonable page loading time, while maintaining the accuracy of the assessment. Upon the comparison between these two image formats, no noticeable differences were found (Figure 14).
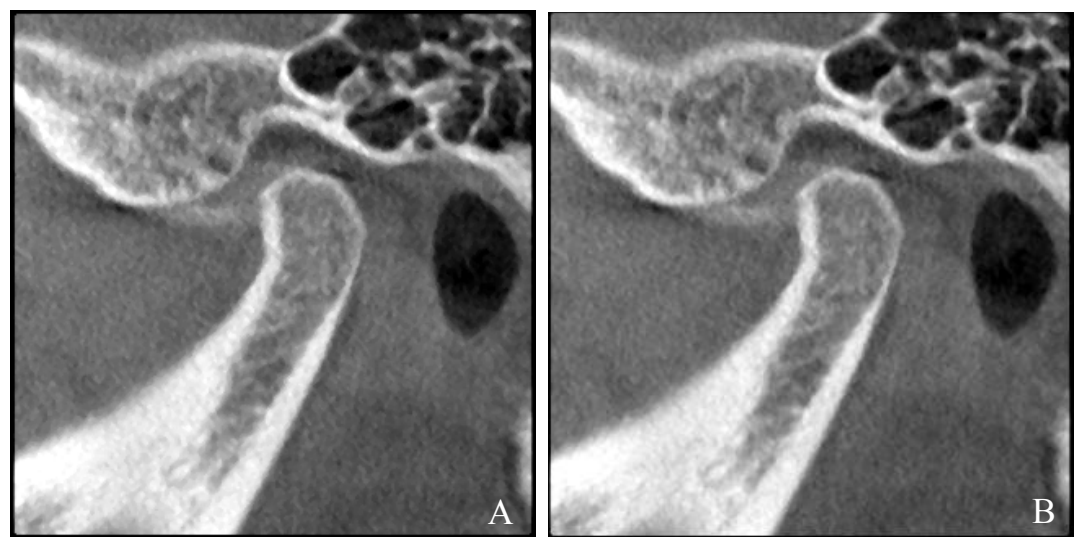

Figure 14. Comparison between (A) A DICOM image, and (B) A converted JPEG image for the TMJ.

On average, the observers' performance in assessing the CBCT examinations was good; however, their performance was lower in certain criteria. Although efforts were made to include all criteria within the examinations, some of the most uncommon criteria were missing. Assessing the size of the abnormalities was challenging, as has been reported in other studies (Marques et al., 2010; Patel et al., 2014). Also, the lack of grading in the extent of osseous changes in the DC/TMD 
image analysis criteria and differentiation between normal morphological variations and small pathological changes (for example, differentiation between thickening of the anterior aspect of the condyle and small osteophyte) could also have affected the performance of the observers, as also stated by Larheim et al. (Larheim et al., 2015). Another challenge lies in distinguishing between a deviation in the shape of the condyle from the normal "rounded" shape without any underlying pathology, such as concavity in the cortical outline, and a deviation associated with pathology. DJD could not be confirmed when only articular surface flattening and/or sclerosis was observed, instead indeterminate for DJD was suggested by Ahmad et al. (Ahmad et al., 2009). Image interpretation is dependent on knowledge, practice and experience, which could explain why no significant difference between results of the two assessments was reported. The educational tools developed in this work are intended for use by general dental practitioners, undergraduate and postgraduate dental students, and specialists for training, and/or improving knowledge on the subject. The web-based programme can be used on a range of devices, including laptops, iPads/tablets, and smartphones. Comparable results have been reported for a web-based programme for training in the anatomical interpretation of maxillofacial CBCT images, and was also very positively received by the observers (Hassan et al., 2007; Al-Rawi et al., 2007). A web-based program for the joint's soft tissue evaluation, developed by Hellen-Halme et al. (Hellen-Halme et al., 2012), can be reviewed for a wider knowledge over the TMJ assessment.

\section{Dose measurements (Study III)}

Although dental radiographic imaging is associated with relatively low radiation doses, the risk arising from ionizing radiation is cumulative, and it is especially important to bear this in mind for children and younger patients. The various organs and tissues in the body differ in their sensitivity to radiation. Undifferentiated and rapidly dividing cells are more radiosensitive which, together with the longer life expectancy of younger patients, stresses the need for extra radiation protection of young people. 
The relevant quantity for planning the exposure of patients, risk-benefit assessments, and potential biochemical changes and physical dose evaluation is the absorbed dose to irradiated tissues (ICRP, 2007). When it is correctly assessed, the organ/tissue absorbed dose correlates with the biological effect of the radiation, including both short-term deterministic and long-term stochastic effects (Fisher and Fahey, 2017). Using the effective dose has several limitations that must be considered when quantifying medical exposure (Brenner, 2008; Brenner, 2012; McCollough et al., 2010). The effective dose represents a generic estimate of the risk arising from a given procedure to a generic model of the human body, and can be of value when comparing risk doses from different diagnostic procedures, but cannot be applied on an individual level (ICRP, 2007). It should not be used for epidemiological studies or the estimation of risks to the population because of the inherent uncertainty and oversimplification involved (McCollough et al., 2010). This is particularly true for children, as the biokinetic models used for the ICRP tabulated weighting factors are typically derived from adult data, and their applicability to children has not yet been ascertained (Fahey et al., 2017). Therefore, risk assessment in medical diagnosis and treatment using ionizing radiation is best evaluated using appropriate risk values for the individual tissues at risk, and for the age and sex of the individuals undergoing these medical procedures (ICRP, 2007). This is why the term "radiation burden" was used in Study III, as it is proportional to the energy imparted.

Dosimeters that can be used to measure absorbed doses in phantoms irradiated in CBCT or panoramic X-ray units include thermoluminescent dosimeters (TLDs), optically stimulated luminescence (OSL) and radiochromic film. The system of choice should have as little energy dependence as possible, and high spatial resolution. The latter requirement is due to the steep dose gradients in $\mathrm{CBCT}$ imaging, it is even more pronounced in panoramic imaging.

The energy distribution of X-rays is affected by the amount of tissue they have passed through. Figure 15 shows the primary X-ray spectra for an Accuitomo 170 unit impinging on the phantom, and after passing through $150 \mathrm{~mm}$ soft tissue and $150 \mathrm{~mm}$ soft tissue $+10 \mathrm{~mm}$ cortical bone. 


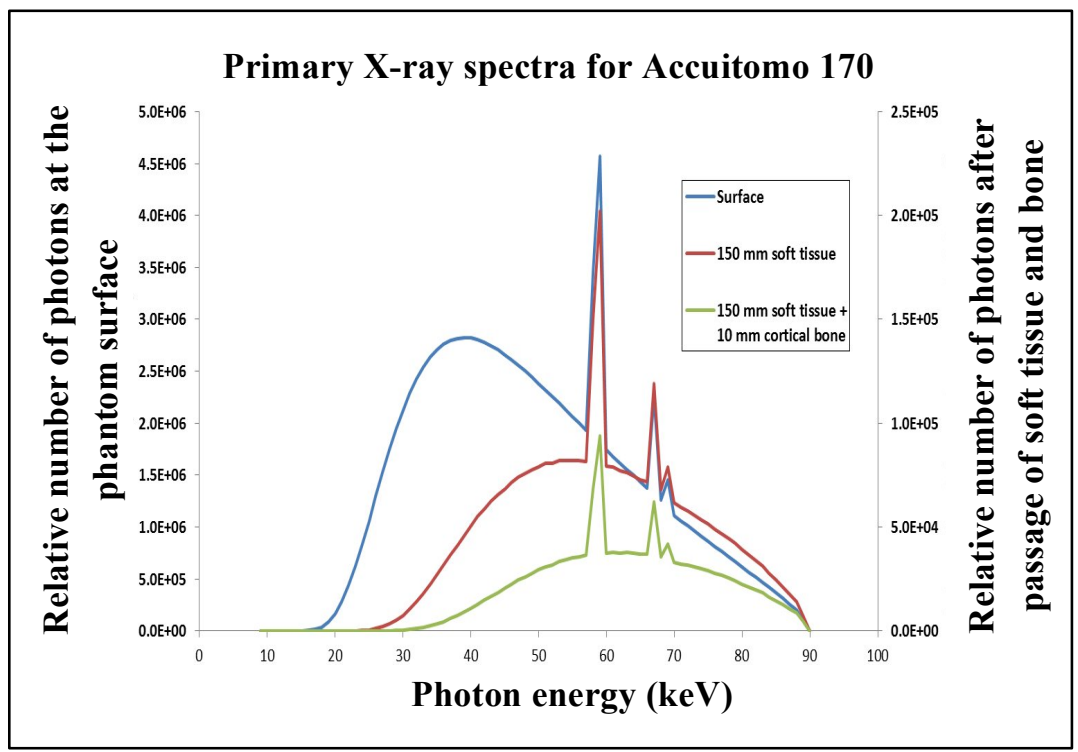

Figure 15. Primary X-ray spectra from an Accuitomo 170 unit at the surface of the phantom, after passing through $150 \mathrm{~mm}$ soft tissue, and after passing through $150 \mathrm{~mm}$ soft tissue $+10 \mathrm{~mm}$ cortical bone.

The spectra were calculated as described previously (Poludniowski et al., 2009). It can be seen that the energy distribution is significantly affected by the tissues, and single and multiple scattering will lead to further differences.

The material of the TLD (LiF:Mg, Ti) has a mean atomic number closest to that of soft tissue, and known as TLD-100. However, due to its slightly higher mean atomic number than that for air, water or soft tissue, the output signal from TLD-100 is significantly dependent on photon energy, as can be seen in Figure 16, which is based on data from Nunn et al. (Nunn et al., 2008). This means that the calibration factor for TLD-100 is dependent on the position in the phantom, and that knowledge of the X-ray spectrum at the measurement point is required to apply the correct calibration factor. The energy dependence is even more pronounced for an OSL system using A12O3:C due to the high atomic number of $\mathrm{Al}$. 


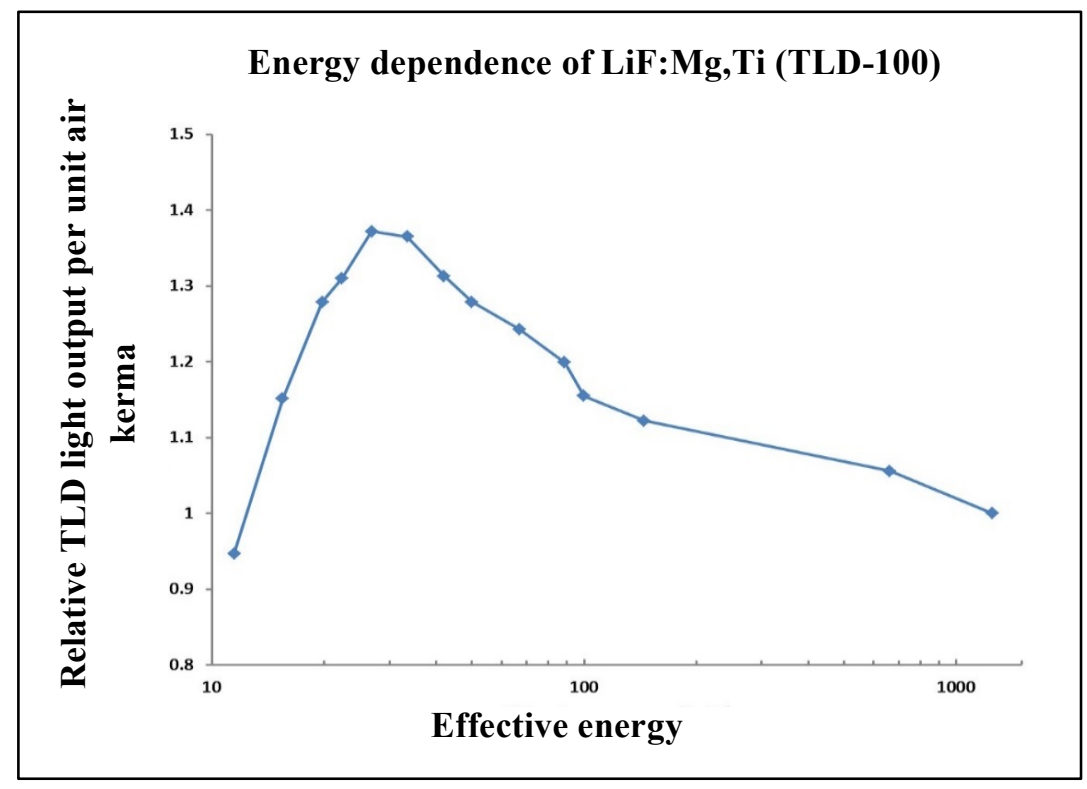

Figure 16. Energy dependence of LiF:Mg,Ti expressed as TLD light output per unit air kerma relative to that for $60 \mathrm{Co}(1250 \mathrm{keV})$.

The radiosensitive material in the radiochromic film used in the present work, Gafchromic XR-QA2, is diacetylene, $\mathrm{C}_{4} \mathrm{H}_{2}$. The composition of the film is near tissue-equivalent (Hourdakis and Nowotny, 2014). The demand for high spatial resolution can be illustrated by the dose distribution obtained from a panoramic examination, shown in Figure 17 , where it can be seen from the distribution of the net grayscale values along the red line that the dose gradients are very steep. A TLD-100 chip is included in the figure, for scaling, to demonstrate the shortcomings of this dosimeter in correctly measuring the true dose values in steep gradients. The tissue equivalence and very high spatial resolution of the Gafchromic film made this a better choice as the dosimeter in Study III. 


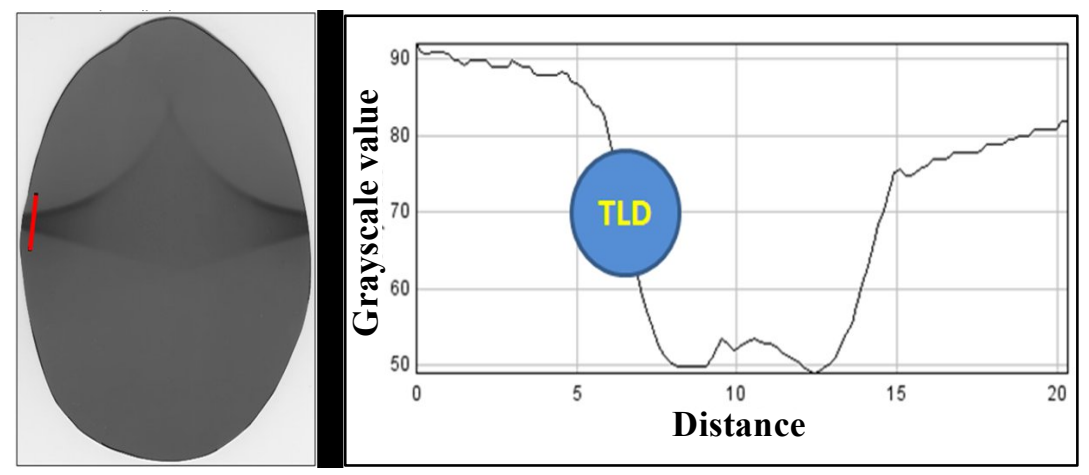

Figure 17. Illustration of the steep dose gradient along the red line in panoramic examination. The size of a typical TLD-100 chip (drawn to scale) is inserted for comparison.

The three X-ray units used in this study had different radiation burdens for both panoramic and CBCT imaging (Figure 10). These differences were found to be related to various factors: exposure parameters (the tube current, voltage and exposure time), the isocentre distance and the total filtration (see Figure 18 and Table 1). The type, size and location of the organ or tissue also affect the absorbed dose. For example, the salivary glands, which are within the rotation centre, were among the organs with the highest absorbed dose, together with the bone surface, which has higher X-ray attenuation than soft tissue. This is in line with previous reports on radiation dosimetry from dental imaging (Nascimento et al., 2017; Kadesjö et al., 2015; Al-Okshi et al., 2013; Ludlow et al., 2006). The rotation angle also contributes to the absorbed dose in a linear way, however, the absorbed doses generally were not halved when using the half-rotation scan instead of the full-rotation scan. That could be related to the rotation of the tube and the position of organs within the FOV for example, the eyes, in relation to the starting angle of a half-rotation scan. In addition that the exposure time in the default setting of half-rotation scan was not halved the time in fullrotation scan. As the three units in this study were very different, and there were differences between the size and location of the organs in the two phantoms, it is not clear whether the doses to the child head phantom should be, in general, larger than those to the adult head phantom as previously reported (Al Najjar et al., 2013; Wahid et al., 2017). 


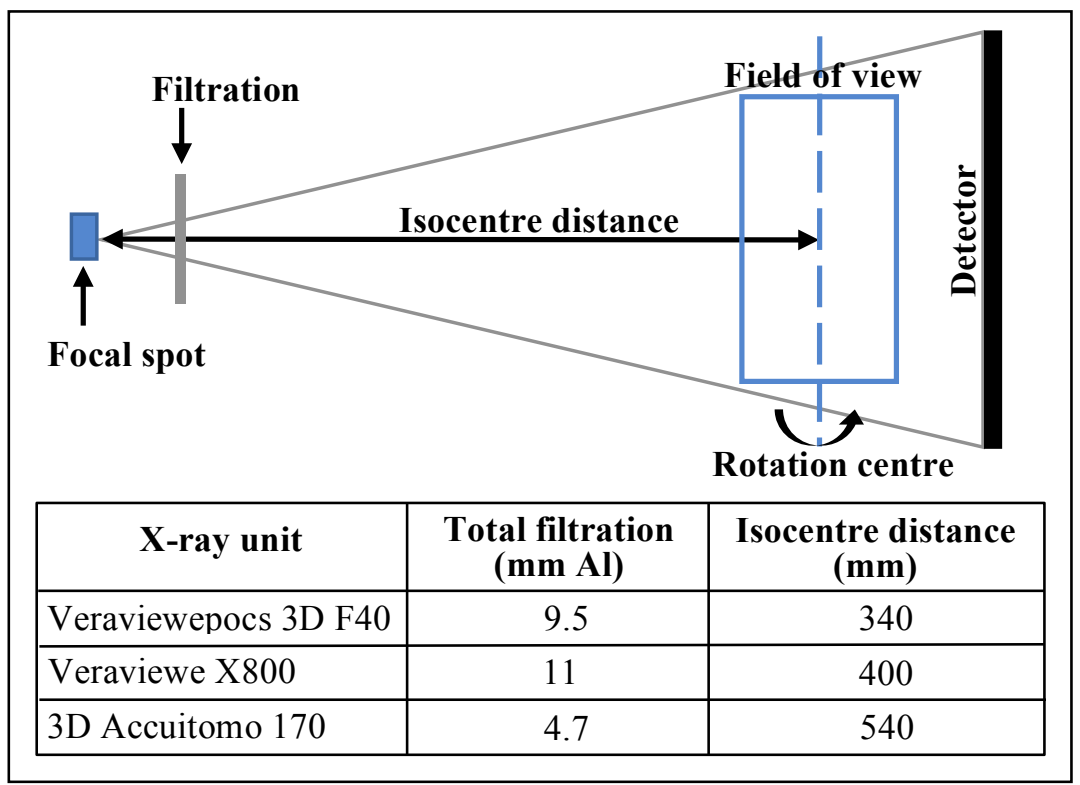

Figure 18. Parameters that affect the radiation burden resulting from the three CBCT units used in Study III.

Several FOVs can be used in a bilateral TMJ CBCT examination, and it is important to use the most appropriate FOV in regard to the radiation dose. Using two small (bilateral) FOVs for the TMJ reduced the irradiated region, and thus decreased the radiation dose to the patient, compared to one large FOV, and will also possibly reduce the time and effort required for image interpretation by the observer.

Uncertainty in dose measurements is unavoidable, and it can be occurred during calibration process, fitting the calibration curve, and/or dose measurement process (Devic, 2011). Maintaining the same set-up during calibration and measurements reduces the uncertainty in dose measurements. The uncertainty in the absorbed dose measured at a single point in Study III was estimated to be $\pm 13 \%$, despite the fact that measurements were carried out over a large number of pixels. The PCXMC Monte Carlo programme (Tapiovaara and Siiskonen, 2008) was used to verify some of the measurements. This is based on 
stochastic mathematical simulation of the interactions between photons and matter. The doses to various organs in patients of different ages and sizes were calculated in freely adjustable X-ray beam projections and other examination conditions that are used in medical X-ray examinations. Good agreement was found (within 3\%) between the measured and calculated mean absorbed dose to the salivary glands for both phantoms from full-rotation CBCT examinations using two different X-ray units.

\section{Dose optimisation (Study IV)}

The radiation dose associated with $\mathrm{CBCT}$ imaging is highly related to the exposure parameters selected by the operator according to pre-set or manually set protocols. Optimisation studies have shown considerable potential for dose reduction in line with the ICRP recommendations (ICRP, 2007). However, knowledge is required on how reductions in dose affect image quality in relation to the diagnostic task. The key to dose optimisation is thus to choose a protocol that provides the lowest radiation dose to the patient, according to the ALADA principle, while providing the information necessary for diagnosis. Selecting a FOV that is limited to the region of interest is an important measure in reducing patient dose, as found in Study III. Several approaches can be used for optimisation (Yeung et al., 2019) and reducing the tube current was evaluated in Study IV. A series of experimental testings were performed using the adult head phantom to identify the tube current suitable for a low-dose protocol. It is common to use phantoms for such studies; however, clinical translation is necessary to ensure that the low-dose protocol is acceptable under clinical conditions. It could be difficult to mimic some of the pathological changes of TMJ in experimental circumstances, besides the radiological interpretation is heavily influenced by the clinical context such as motion artifacts. Therefore, resultant images from experimental testing could not be directly corresponding to the images under the clinical condition. Also, the observer's clinical experience in image interpretation could be another challenge, therefore, the diagnostic accuracy of the low-dose protocol could not be guaranteed under the experimental testing only. The Regional Ethics Review Board in Lund deemed that the potential benefit in future clinical practice in CBCT examinations of the TMJ was greater 
than the increased radiation burden to the patients included in Study IV, which was equivalent to the background radiation during less than one week in Sweden.

Different diagnostic tasks require different levels of image quality, as stated by both the ICRP (ICRP, 2007) and the European Commission (European Commission, 2012). For instance, implant planning and periapical diagnosis require different levels of image quality. Therefore, imaging performance should be assessed bearing in mind the clinical question (Lofthag-Hansen et al., 2011; Choi et al., 2015; Choi, 2016; Park, 2019). In the present context, it should be possible to detect osseous changes when any form of DJD is suspected; thus, lower radiation dose protocols could be possible. A limited number of studies have been published on CBCT protocol optimisation for the TMJ. Kadesjö et al. (Kadesjö et al., 2015) reported the effectiveness of a lowdose protocol with a $50 \%$ reduction in the tube current, while Yadav et al. (Yadav et al., 2015) reported the usefulness of a $180^{\circ} \mathrm{CBCT}$ acquisition protocol for TMJ arthritic lesions, compared to $360^{\circ}$ acquisition. The findings of the present study are in line with these previous findings, and highlight the fact that the diagnostic task should be considered when choosing the exposure parameters for CBCT scans of the TMJ. The low-dose protocol developed in this study, in which the dose was only $20 \%$ of that recommended in the manufacturer's protocol, provided adequate image quality for the evaluation of the TMJ, without any significant difference in the diagnostic accuracy.

When the diagnostic performance is influenced by high levels of noise, a noise-reduction algorithm can be used to improve the image quality. Such algorithms filter out the noise to varying degrees, while preserving the texture, contour, edges and fine details (Rudin et al., 1992). The noise-reduction method used in Study IV has been reported to be the technique of choice when very fine details are not required and the observers are interested mainly in the anatomical features (Shahmoradi et al., 2016). However, as the diagnostic task was not significantly influenced by reducing the tube current, noise reduction did not significantly improve the diagnostic accuracy of the CBCT examination (Table 3). 
Younger individuals in whom developing tissues are more radiosensitive are at higher risk from X-ray exposure than older people. Moreover, since younger patients exhibit an increased prevalence of TMD (National Institute of Dental and Craniofacial Research), it is especially important to optimise the CBCT scans of the TMJ as much as possible in this age group. A low-dose protocol should therefore be the obvious choice for younger patients. However, it must be borne in mind that the low-dose protocol evaluated in Study IV is specific to the X-ray unit used (Veraviewepocs 3D F40), and the findings cannot be generalized to other units without further investigation. Eventually, there is an urgent need to evaluate protocols for CBCT examinations of the TMJ to optimise them to ensure that the radiation dose is as low as diagnostically acceptable (the ALADA principle recommended by NCRP).

\section{Implementation of the results}

The findings presented in this thesis were applied in dental clinical practice at Malmö University. The CBCT educational tools presented in Studies I and II are now recommended to learn the radiological interpretation of TMJ. Furthermore, the use of two small FOVs for TMJ bilateral evaluation is emphasized, (Study III). The low-dose protocol that was evaluated in Study IV for CBCT imaging of the TMJ is now applied, particularly for young patients, as a dose optimisation measure. 


\section{CONCLUSIONS}

- The educational tool presented in Study I was a useful tool for the assessment of TMJ osseous tissues using CBCT images (Study I).

- The web-based educational programme that was developed and then tested by dental students could be a useful educational tool for the interpretation of CBCT images of the TMJ (Study II).

- Both CBCT educational tools can be used in education of general dental practitioners, undergraduate/postgraduate dental students, and specialists (Study I and II).

- The mean absorbed dose within and between radiosensitive organs and tissues (the brain, eyes, salivary glands, oral mucosa and bone surface) varied considerably between the adult and child phantoms. The bone surface and salivary glands received the highest absorbed doses, both in panoramic radiography and CBCT examination of the TMJ (Study III).

- In bilateral CBCT examination of the TMJ, two small FOVs (4 $\mathrm{x} 4 \mathrm{~cm}$ ) led to a lower absorbed dose than one large FOV (14 x $5 \mathrm{~cm}$ ), (Study III).

- For the CBCT unit used in Study IV (Veraviewepocs 3D F40), the low-dose protocol for CBCT examination of the TMJ was diagnostically comparable to the manufacturer-recommended protocol, despite the fact that the radiation dose was five times lower (Study IV). 


\section{FUTURE RESEARCH}

As CBCT has been widely accepted as an imaging tool in various dental applications, there is a need for more research and studies to improve and optimise using the technology on different levels in the dental field. The underlying purpose is to provide better and more evidence-based patient care.

- $\quad$ Since the educational tools were well-received by the participated observers, more radiographic educational tools should be developed to cover different diagnostic tasks in order to increase knowledge on the interpretation of CBCT images.

- $\quad$ Few studies have investigated the feasibility of using Gafchromic films for dose measurements in dental diagnostic radiology. Further investigations and comparisons with other dosimetric methods, such as TLDs and OSL are thus desired.

- The possibility of implementing low-dose protocols in CBCT examinations should be investigated, as well as implementing the proposed protocol in other CBCT units.

- Transdisciplinary collaboration, including oral radiologists and medical physicists, as recommended by the international radiation protection community, is necessary to optimise the settings of CBCT units, to ensure sufficient image quality that provide answers to relevant clinical questions, while ensuring the lowest possible radiation dose to the patient, according to the ALADA principle. 


\section{ACKNOWLEDGEMENTS}

First and foremost, praise be to God the Almighty for the successful completion of this thesis.

I would like to extend my sincere thanks and appreciation to all those without whom I would not have been able to complete this work. My very special thanks and sincere gratitude go to:

Associate Professor Kristina Hellén-Halme, my principal supervisor, for believing in me, for providing endless guidance and encouragement, and for supporting and helping throughout my journey. It has been an honour working with you.

I am also sincerely grateful to Professor Mats Nilsson, who was a wonderful teacher, and supporter throughout my work. He provided constant help and guidance. I cannot find the words to describe how valuable your role was.

I would also like to express my sincere thanks to Professor Per Alstergren for his help, as well as his valuable contributions to my work.

In addition, I would like to thank Professor Xieqi Shi for participating in, and critically revising Studies II and IV, and Professor Ewa Carin Ekberg for participating in and critically revising Studies II.

Also, many thanks to Dr. Karin Näsström, Dr. Lars-Göran Hansson and Professor Arne Petersson for their participation as observers in the studies.

Many thanks to Radiographer Curt Johansson for his help in the clinical part of my studies. 
Special thanks are extended to King Abdulaziz University, Jeddah, Saudi Arabia, for providing my $\mathrm{PhD}$ scholarship, and for supporting me throughout my studies at Malmo University.

Many thanks to all my colleagues at the Department of Oral Radiology, for embracing me as a member of their big family.

I would very much like to thank my sister Aisha Iskanderani for her help with the illustrations and figures included in the papers and this thesis.

Special thanks to my parents and siblings for being in my life, and for their valuable and continuous support.

Last, but not least, I would like especially to thank, and express my sincere love and appreciation to, my husband Hussien, for his patience and encouragement, and for always believing in me and giving me all the support I needed. My thanks to my lovely daughters, Maya and Malak, who fill my life with joy and happiness. 


\section{REFERENCES}

Ahmad M, Hollender L, Anderson Q, Kartha K, Ohrbach R, Truelove EL, et al. (2009) Research diagnostic criteria for temporomandibular disorders (RDC/TMD): development of image analysis criteria and examiner reliability for image analysis. Oral Surg Oral Med Oral Pathol Oral Radiol Endod 107:844-60.

Al Najjar A, Colosi D, Dauer LT, Prins R, Patchell G, Branets I, et al. (2013) Comparison of adult and child radiation equivalent doses from 2 dental cone-beam computed tomography units. Am J Orthod Dentofacial Orthop 143:784-92.

Al-Okshi A, Nilsson M, Petersson A, Wiese M, Lindh C. (2013) Using GafChromic film to estimate the effective dose from dental cone beam CT and panoramic radiography. Dentomaxillofac Radiol 42:20120343.

Al-Rawi WT, Jacobs R, Hassan BA, Sanderink G, Scarfe WC. (2007) Evaluation of web-based instruction for anatomical interpretation in maxillofacial cone beam computed tomography. Dentomaxillofac Radiol 36:459-64.

Altman DG. (1991) Practical statistics for medical research. 1st ed. London, UK: Chapman and Hall.

Arai Y, Tammisalo E, Iwai K, Hashimoto K, Shinoda K. (1999) Development of a compact computed tomographic apparatus for dental use. Dentomaxillofac Radiol 28:245-8.

Boeddinghaus R, Whyte A. (2013) Computed tomography of the temporomandibular joint. J Med Imaging Radiat Oncol 57:448-54.

Brenner DJ. (2008) Effective dose: a flawed concept that could and should be replaced. Br J Radiol 81:521-3. 
Brenner DJ. (2012) We can do better than effective dose for estimating or comparing low-dose radiation risks. Ann ICRP 41:124-8.

Buchanan A, Thachil K, Haggard C, Kalathingal S. (2017) Predoctoral and Postdoctoral Education on Cone-Beam Computed Tomography. $J$ Evid Based Dent Pract 17:310-6.

Bushberg JT. (2014) Science, Radiation Protection and the NCRP: Building on the Past, Looking to the Future. In: Proceedings of the fiftieth annual meeting program of NCRP: achievements of the past 50 years and addressing the needs of the future, March 10-11. Bethesda, MD. pp. 5-7.

Butson MJ, Yu PKN, Cheung T, Alnawaf H. (2010) Energy response of the new EBT2 radiochromic film to x-ray radiation. Radiat Meas 45:836-9.

Carlsson GA, Dance DR, Persliden J, Sandborg M. (1999) Use of the concept of energy imparted in diagnostic radiology. Appl Radiat Isot 50:39-62.

Choi J-W. (2016) Analysis of the priority of anatomic structures according to the diagnostic task in cone-beam computed tomographic images. Imaging Sci Dent 46:245-9.

Choi J-W, Lee S-S, Choi S-C, Heo M-S, Huh K-H, Yi W-J, et al. (2015) Relationship between physical factors and subjective image quality of cone-beam computed tomography images according to diagnostic task. Oral Surg Oral Med Oral Pathol Oral Radiol 119:357-65.

Clark GT. (2001) Education problems and Web-based teaching: how it impacts dental educators? J Am Coll Dent 68:25-34.

Dance DR, Castellano I. (2014) Patient Dosimetry. In Dance DR, Christofides S, Maidment ADA, McLean ID, Ng KH (Eds). Diagnostic radiology physics: a handbook for teachers and students. Endorsed by: American Association of Physicists in Medicine, Asia-Oceania Federation of Organizations for Medical Physics, European Federation 
of Organisations for Medical Physics. Vienna, Austria: International Atomic Energy Agency.

Das IJ. (2017) Radiochromic film: role and applications in radiation dosimetry: CRC Press.

Devic S. (2011) Radiochromic film dosimetry: past, present, and future. Phys Med 27:122-34.

Dworkin SF, LeResche L. (1992) Research diagnostic criteria for temporomandibular disorders: review, criteria, examinations and specifications, critique. J Craniomandib Disord 6:301-55.

European Commission. (2012) Cone beam CT for dental and maxillofacial radiology: Evidence-based guidelines, Radiation $\begin{array}{lll}\text { protection } & \text { publication } & \text { [Retrieved from }\end{array}$ http://www.sedentexct.eu/files/radiation_protection_172.pdf].

Fahey FH, Goodkind AB, Plyku D, Khamwan K, O'Reilly SE, Cao X, et al. (2017) Dose Estimation in Pediatric Nuclear Medicine. Semin Nucl Med 47:118-25.

Fisher DR, Fahey FH. (2017) Appropriate Use of Effective Dose in Radiation Protection and Risk Assessment. Health Phys 113:102-9.

Francis B, Mauriello SM, Phillips C, Englebardt S, Grayden SK. (2000) Assessment of online continuing dental education in North Carolina. $J$ Contin Educ Health Prof 20:76-84.

Hashimoto K, Arai Y, Iwai K, Araki M, Kawashima S, Terakado M. (2003) A comparison of a new limited cone beam computed tomography machine for dental use with a multidetector row helical CT machine. Oral Surg Oral Med Oral Pathol Oral Radiol Endod 95:3717.

Hashimoto K, Kawashima S, Kameoka S, Akiyama Y, Honjoya T, Ejima K, et al. (2007) Comparison of image validity between cone beam 
computed tomography for dental use and multidetector row helical computed tomography. Dentomaxillofac Radiol 36:465-71.

Hassan B, Jacobs R, Scarfe WC, Al-Rawi WT. (2007) A web-based instruction module for interpretation of craniofacial cone beam CT anatomy. Dentomaxillofac Radiol 36:348-55.

Hellen-Halme K, Hollender L, Janda M, Petersson A. (2012) Web-based calibration of observers using MRI of the temporomandibular joint. Dentomaxillofac Radiol 41:656-61.

Hellen-Halme K, Nilsson M. (2013) The Effects on Absorbed Dose Distribution in Intraoral X-ray Imaging When Using Tube Voltages of 60 and $70 \mathrm{kV}$ for Bitewing Imaging. J Oral Maxillofac Res 4:e2.

Honda K, Larheim TA, Maruhashi K, Matsumoto K, Iwai K. (2006) Osseous abnormalities of the mandibular condyle: diagnostic reliability of cone beam computed tomography compared with helical computed tomography based on an autopsy material. Dentomaxillofac Radiol $35: 152-7$.

Horner K, Islam M, Flygare L, Tsiklakis K, Whaites E. (2009) Basic principles for use of dental cone beam computed tomography: consensus guidelines of the European Academy of Dental and Maxillofacial Radiology. Dentomaxillofac Radiol 38:187-95.

Hourdakis J, Nowotny R. (2014) Instrumentation for Dosimetry. In Dance DR, Christofides S, Maidment ADA, McLean ID, Ng KH (Eds). Diagnostic radiology physics: A handbook for teachers and students Endorsed by: American Association of Physicists in Medicine, AsiaOceania Federation of Organizations for Medical Physics, European Federation of Organisations for Medical Physics. Vienna, Austria: International Atomic Energy Agency.

Howerton WB, Enrique PRT, Ludlow JB, Tyndall DA. (2004) Interactive computer-assisted instruction vs. lecture format in dental education. $J$ Dent Hyg 78:10. 
Iskanderani D, Alstergren P, Hellén-Halme K (2018) Assessment of a training programme on detection of temporomandibular joint osseous changes applying pre-defined 2D multiplane cone beam computed tomography reconstructions. J Oral Rehabil 45:282-8.

Kadesjo N, Benchimol D, Falahat B, Nasstrom K, Shi XQ. (2015) Evaluation of the effective dose of cone beam computed tomography and multi-slice computed tomography for temporomandibular joint examinations at optimized exposure levels. Dentomaxillofac Radiol 20150041.

Kaimal S, Ahmad M, Kang W, Nixdorf D, Schiffman EL, Schiffman EL. (2018) Diagnostic accuracy of panoramic radiography and MRI for detecting signs of TMJ degenerative joint disease. Gen Dent 66:34-40.

Larheim TA, Abrahamsson A-K, Kristensen M, Arvidsson LZ. (2015) Temporomandibular joint diagnostics using CBCT. Dentomaxillofac Radiol 44:20140235.

Lavanya R, Babu DB, Waghray S, Chaitanya NC, Mamatha B, Nithika M. (2016) A Questionnaire Cross-Sectional Study on Application of CBCT in Dental Postgraduate Students. Pol J Radiol 81:181-9.

Leon SD. (2004) The use of panoramic radiography for evaluating temporomandibular disorders. Gen Dent 52:339-41.

Lindfors E, Tegelberg Å, Magnusson T, Ernberg M. (2016) Treatment of temporomandibular disorders - knowledge, attitudes and clinical experience among general practising dentists in Sweden. Acta Odontol Scand 74:460-5.

Lofthag-Hansen S, Thilander-Klang A, Gröndahl K. (2011) Evaluation of subjective image quality in relation to diagnostic task for cone beam computed tomography with different fields of view. Eur $J$ Radiol $80: 483-8$. 
Ludlow JB, Davies-Ludlow LE, Brooks SL, Howerton WB. (2006) Dosimetry of 3 CBCT devices for oral and maxillofacial radiology: $\mathrm{CB}$ Mercuray, NewTom 3G and i-CAT. Dentomaxillofac Radiol 35:219-26.

Marques AP, Perrella A, Arita ES, Pereira MF, Cavalcanti Mde G. (2010) Assessment of simulated mandibular condyle bone lesions by cone beam computed tomography. Braz Oral Res 24:467-74.

Mawani F, Lam EW, Heo G, McKee I, Raboud DW, Major PW. (2005) Condylar shape analysis using panoramic radiography units and conventional tomography. Oral Surg Oral Med Oral Pathol Oral Radiol Endod 99:341-8.

McCollough CH, Christner JA, Kofler JM. (2010) How effective is effective dose as a predictor of radiation risk? AJR Am J Roentgenol 194:890-6.

Miracle AC, Mukherji SK. (2009) Conebeam CT of the head and neck, part 1: physical principles. AJNR Am J Neuroradiol 30:1088-95.

Mitov G, Dillschneider T, Abed MR, Hohenberg G, Pospiech P. (2010) Introducing and evaluating MorphoDent, a Web-based learning program in dental morphology. J Dent Educ 74:1133-9.

Mozzo P, Procacci C, Tacconi A, Martini PT, Andreis IA. (1998) A new volumetric CT machine for dental imaging based on the cone-beam technique: preliminary results. Eur Radiol 8:1558-64.

Nascimento HAR, Andrade MEA, Frazão MAG, Nascimento EHL, Ramos-Perez FMM, Freitas DQ. (2017) Dosimetry in CBCT with Different Protocols: Emphasis on Small FOVs Including Exams for TMJ. Braz Dent J 28:511-6.

National Institute of Dental and Craniofacial Research. Prevalence of TMJD and its Signs and Symptoms. [Retrieved from https://www.nidcr.nih.gov/research/data-statistics/facialpain/prevalence]. 
Neves FS, de Camargo Souza T, de-Azevedo-Vaz SL, Campos PSF, Bóscolo FN. (2014) Influence of cone-beam computed tomography milliamperage settings on image quality of the mandibular third molar region. Oral Radiol 30:27-31.

Nunn AA, Davis SD, Micka JA, DeWerd LA. (2008) LiF:Mg,Ti TLD response as a function of photon energy for moderately filtered x-ray spectra in the range of $20-250 \mathrm{kVp}$ relative to $60 \mathrm{Co}$. Med Phys 35:185969.

Ohrbach R, Dworkin SF. (2016) The Evolution of TMD Diagnosis: Past, Present, Future. J Dent Res 95:1093-101.

Park H-N, Min C-K, Kim K-A, Koh K-J. (2019) Optimization of exposure parameters and relationship between subjective and technical image quality in cone-beam computed tomography. Imaging Sci Dent 49:139-51.

Patel A, Tee BC, Fields H, Jones E, Chaudhry J, Sun Z. (2014) Evaluation of cone-beam computed tomography in the diagnosis of simulated small osseous defects in the mandibular condyle. Am J Orthod Dentofacial Orthop 145:143-56.

Pauwels R, Seynaeve L, Henriques JCG, de Oliveira-Santos C, Souza PC, Westphalen FH, et al. (2015) Optimization of dental CBCT exposures through mAs reduction. Dentomaxillofac Radiol 44:20150108.

Pauwels R, Silkosessak O, Jacobs R, Bogaerts R, Bosmans H, Panmekiate S. (2014) A pragmatic approach to determine the optimal $\mathrm{kVp}$ in cone beam CT: balancing contrast-to-noise ratio and radiation dose. Dentomaxillofac Radiol 43:20140059.

Peck CC, Goulet J-P, Lobbezoo F, Schiffman EL, Alstergren P, Anderson GC, et al. (2014) Expanding the taxonomy of the diagnostic criteria for temporomandibular disorders. J Oral Rehabil 41:2-23. 
Petersson A. (2010) What you can and cannot see in TMJ imaging--an overview related to the RDC/TMD diagnostic system. $J$ Oral Rehabil 37:771-8.

Poludniowski G, Landry G, DeBlois F, Evans PM, Verhaegen F. (2009) SpekCalc: a program to calculate photon spectra from tungsten anode $\mathrm{x}-$ ray tubes. Phys Med Biol 54:N433-8.

Rampado O, Bianchi SD, Cornetto AP, Rossetti V, Ropolo R. (2014) Radiochromic films for dental CT dosimetry: a feasibility study. Phys Med 30:18-24.

Reiser I. (2014) Image Perception and Assessment. In Dance DR, Christofides S, Maidment ADA, McLean ID, Ng KH (Eds). Diagnostic radiology physics: A handbook for teachers and students Endorsed by: American Association of Physicists in Medicine, Asia-Oceania Federation of Organizations for Medical Physics, European Federation of Organisations for Medical Physics. Vienna, Austria: International Atomic Energy Agency.

Rudin LI, Osher S, Fatemi E. (1992) Nonlinear total variation based noise removal algorithms. Physica D 60:259-68.

Scarsbrook AF, Graham RN, Perriss RW. (2005) The scope of educational resources for radiologists on the internet. Clin Radiol 60:524-30.

Schiffman E, Ohrbach R, Truelove E, Look J, Anderson G, Goulet JP, et al. (2014) Diagnostic Criteria for Temporomandibular Disorders (DC/TMD) for Clinical and Research Applications: recommendations of the International RDC/TMD Consortium Network* and Orofacial Pain Special Interest Group $\dagger$. J Oral Facial Pain Headache 28:6-27.

Schmitter M, Gabbert O, Ohlmann B, Hassel A, Wolff D, Rammelsberg P, et al. (2006) Assessment of the reliability and validity of panoramic imaging for assessment of mandibular condyle morphology using both MRI and clinical examination as the gold standard. Oral Surg Oral Med Oral Pathol Oral Radiol Endod 102:220-4. 
SEDENTEXCT Project. CBCT Training Programme: Interpretation of pathology on CBCT images. [Retrieved from http://www.sedentexct.eu/training/m10/m10_intro.htm].

Shahmoradi M, Lashgari M, Rabbani H, Qin J, Swain M. (2016) A comparative study of new and current methods for dental micro-CT image denoising. Dentomaxillofac Radiol 45:20150302.

Sirin Y, Guven K, Horasan S, Sencan S. (2010) Diagnostic accuracy of cone beam computed tomography and conventional multislice spiral tomography in sheep mandibular condyle fractures. Dentomaxillofac Radiol 39:336-42.

Sur J, Seki K, Koizumi H, Nakajima K, Okano T. (2010) Effects of tube current on cone-beam computerized tomography image quality for presurgical implant planning in vitro. Oral Surg Oral Med Oral Pathol Oral Radiol Endod 110:e29-33.

Tanaka E, Detamore MS, Mercuri LG. (2008) Degenerative disorders of the temporomandibular joint: etiology, diagnosis, and treatment. $J$ Dent Res 87:296-307.

Tapiovaara M, Siiskonen T. (2008) PCXMC, A Monte Carlo program for calculating patient doses in medical x-ray examinations. $2^{\text {nd }} e d$. Helsinki, Finland: Radiation and Nuclear Safety Authority; Report STUK-A231.

Wahid MA, Choi E, MacDonald DS, Ford NL. (2017) Dosimetry analysis of panoramic_imaging devices in different_sized phantoms. $J$ Appl Clin Med Phys 18:197-205.

Watanabe Y, Patel GN, Patel P. (2006) Evaluation of a new selfdeveloping instant film for imaging and dosimetry. Radiat Prot Dosimetry 120:121-4.

Yadav S, Palo L, Mahdian M, Upadhyay M, Tadinada A. (2015) Diagnostic accuracy of 2 cone-beam computed tomography protocols 
for detecting arthritic changes in temporomandibular joints. Am $J$ Orthod Dentofacial Orthop 147:339-44.

Yeung AWK, Jacobs R, Bornstein MM. (2019) Novel low-dose protocols using cone beam computed tomography in dental medicine: a review focusing on indications, limitations, and future possibilities. Clin Oral Ivestig 23:2573-81.

Zain-Alabdeen EH, Alsadhan RI. (2012) A comparative study of accuracy of detection of surface osseous changes in the temporomandibular joint using multidetector $\mathrm{CT}$ and cone beam CT. Dentomaxillofac Radiol 41:185-91. 
PAPERS I-IV 

I 



\title{
Assessment of a training programme on detection of temporomandibular joint osseous changes applying pre- defined 2D multiplane cone beam computed tomography reconstructions
}

\author{
D. Iskanderani ${ }^{1,2}$ (i) | P. Alstergren ${ }^{3} \mid$ K. Hellén-Halme ${ }^{1}$
}

${ }^{1}$ Department of Oral and Maxillofacial Radiology, Faculty of Odontology, Malmö University, Malmö, Sweden

${ }^{2}$ Department of Oral and Maxillofacial Radiology, Faculty of Dentistry, King Abdulaziz University, Jeddah, Saudi Arabia

${ }^{3}$ Department of Orofacial Pain and Jaw Function, Faculty of Odontology, Malmö University, Malmö, Sweden

\section{Correspondence}

Durer Iskanderani, Department of Oral and Maxillofacial Radiology, Faculty of Odontology, Malmö University, Malmö, Sweden.

Email: durer.iskanderani@mah.se

Funding information

This study was funded by King Abdulaziz University

\begin{abstract}
Summary
Objectives: Osseous changes in temporomandibular joint disorders (TMD) are common indications for cone beam computed tomography (СВCT). The number of such cases is increasing while a number of qualified oral radiologists is limited. This study investigated the usefulness of a training programme for general dental practitioners (GDPs) regarding temporomandibular joint (TMJ) osseous changes detection in CBCT images.

Methods: We selected CBCT images of 35 TMD cases and chose 5 of them to use in training 7 GDPs on detecting osseous changes in the TMJ. In evaluation directly following training, GDPs assessed the 30 remaining cases. Three qualified radiologists served as reference standard. A 2-month follow-up of training comprising evaluation of the same cases. The cases are assessed according to Ahmad et al (Oral Surg Oral Med Oral Pathol Oral Radiol Endod, 107, 2009, 844) protocol of TMJ osseous changes in CBCT images.

Results: Sensitivity and specificity of the CBCT protocol were high, except for some criteria that did not appear in the cases. Average observer sensitivity was $65 \%$ while specificity was $87 \%$. Average correct individual response rate was $84 \%$. Mean agreement among the GDPs was $73 \%$. Observer performance had improved at the 2 -month follow-up.

Conclusion: The present educational programme could be a helpful material on recognising possible osseous changes of TMJ and it can be used as a part of a training programme for GDP and for specialist candidates.

KEYWORDS

dental radiography, education, general practitioners, three-dimensional imaging, temporomandibular joint disorders, volumetric computed tomography
\end{abstract}

\section{1 | INTRODUCTION}

Comprehensive examination and careful diagnosis are crucial steps in treatment plan preparation for temporomandibular disorders (TMD).
The primary assessment tool is often the clinical examination. ${ }^{1}$ But it is usually considered insufficient and unreliable for diagnosing the condition of the temporomandibular joint (TMJ), ${ }^{2}$ particularly if the clinical examination or patient history indicates a recent or progressive 
pathologic joint condition. ${ }^{3}$ Thus, after a thorough history and clinical examination, current recommendations are supplementary radiographic imaging when imaging can be suspected to have a substantial impact on the clinician's diagnosis and treatment approach. ${ }^{3}$

Several studies reported a high diagnostic accuracy of cone beam computed tomography (CBCT) for hard tissues of the maxillofacial region while substantially decreasing radiation dose and cost when compared to conventional $\mathrm{CT}^{4-6}$ Recently, radiologists have described hard tissue abnormalities of the TMJ using $\mathrm{CBCT}^{7-9}$ Demand for CBCT interpretation is also growing as general dental practitioners (GDPs) experience increasing accessibility to $\mathrm{CBCT}$ imaging. The number of qualified radiologists, however, has not kept pace with the demand for interpretation. Horner et $\mathrm{al}^{10}$ reported that even primary dental care centres that lack staff radiologists have purchased CBCT scanners. Because hard tissue abnormalities are difficult to distinguish and can easily be misinterpreted in 3D images, particularly in the complex TMJ region, radiology training is imperative. Qualified radiologists should therefore engage themselves to train the general practitioner in the evaluation of $\mathrm{CBCT}$ examinations.

Various $\mathrm{CBCT}$ training programmes and materials regarding dose optimisation, indications, anatomy and interpretation are available as educational tools. ${ }^{11-14}$ Assessments of some of these reported a high efficacy with the participants and users emphasising the need for such courses to remain conversant with technological advances in patient care. ${ }^{14}$ For the TMJ region, however, training materials are sparse. Thus, this study investigated the usefulness of a training programme for GDPs, but was restricted to detecting TMJ osseous changes in CBCT images and evaluated the GDPs' performance in comparison with oral and maxillofacial radiologists. A further aim was to investigate GDPs' agreement following the training programme.

\section{2 | METHODS}

Cone beam computed tomography images of $35 \mathrm{TMJ}$ cases in DICOM format were randomly selected from the database at the Faculty of Odontology, Malmö University, Malmö, Sweden between 2013 and 2014. The CBCT images were anonymised. According to the radiographic reports, no changes were found in 6 cases while the remainder exhibited varying degrees of osseous changes. The 35 selected cases were uncomplicated (no suspicions of underlying disease or malignancy). One author (DI) made 2 power point (ppt) files in collaboration with an oral and maxillofacial radiologist. The first file, the training programme, contained 5 TMD cases for use in training GDPs how to recognise osseous changes in the TMJ. The second file, the evaluation programme, contained 30 TMD cases to be evaluated by the GDPs who had participated in the training session. This study required no ethics approval.

\section{1 | Radiographic examination}

The CBCT images had been exposed with a 3D Accuitomo 170 CBCT scanner (J Morita Corp, Kyoto, Japan) with each scan acquired in a $180^{\circ}$ rotation with the same acquisition parameters ( $80 \mathrm{KvP}, 5 \mathrm{~mA}, 9.4 \mathrm{~s}$ ). Multiplanar reconstructions (MPR) sagittal, axial and coronal were generated. The pixel size was $0.125 \times 0.125 \mathrm{~mm}^{2}$. For each case, we selected 3 MPR images and exported them to the Power Point (PPT) files for the training and evaluation programmes. The 2 PPT files can be accessed at http://mah.se/fakulteter-och-omraden/Odontologiska-fakulteten/ Avdelning-och-kansli/Odontologisk-rontgendiagnostik/TMJ1/.

\section{2 | Observers}

This study included 7 international GDPs from various countries and was conducted at Malmö University, Faculty of Odontology, Malmö, Sweden. None of the GDPs had had previous training in CBCT imaging technique.

\subsection{1 | Training programme}

The training and calibration programme began with a brief description of the anatomy of healthy TMJ region using illustrations and figures. We presented a protocol (Table 1) that detailed various osseous changes which could occur in CBCT images of the TMJ. The protocol was the 1 published by Ahmad et al, ${ }^{15}$ which we supplemented with criteria definitions and figures. The programme included 5 cases of suspected TMD: 4 had a variety of osseous changes, and 1 case exhibited no osseous changes. One author (DI) accompanied the GDPs during the training programme.

\subsection{2 | Evaluation programme}

Directly after completing the training programme, on the same day, the GDPs evaluated the 30 cases of suspected TMD. Each case comprised the most representative corrected sagittal, coronal and axial images (Figure 1). The reformatted images were prepared in advance by the authors. The participants interpreted the images independently on computer monitors. All observers used the same computer and the same monitor. The illuminance in the room was kept at the same level for all evaluations. A protocol was filled out for each case. Image viewing was not time-limited.

Three qualified radiologists (each with more than 10 years of experience in evaluating TMJs on CBCT images) evaluated the 30 cases together at the same occasion. Their consensus served as the reference standard. At a 2-month follow-up, the GDPs re-evaluated the cases. During this period, the observers did not work with radiographic evaluation including $\mathrm{CBCT}$ examinations.

\section{3 | Radiographic evaluation}

The protocol provided definitions for all criteria and allowed only dichotomous responses (Yes/No) for each of the criteria for the presence of osseous changes in the TMJ. The images for each case were evaluated in the following sequence: changes of condylar head, articular eminence and condylar position. The radiologists and GDPs were blinded to the clinical history and the previous diagnosis of the selected cases. 
TAB LE 1 Cone beam computed tomography protocol for the assessment of osseous changes of temporomandibular joint according to the criteria of Ahmad et $\mathrm{al}^{15}$

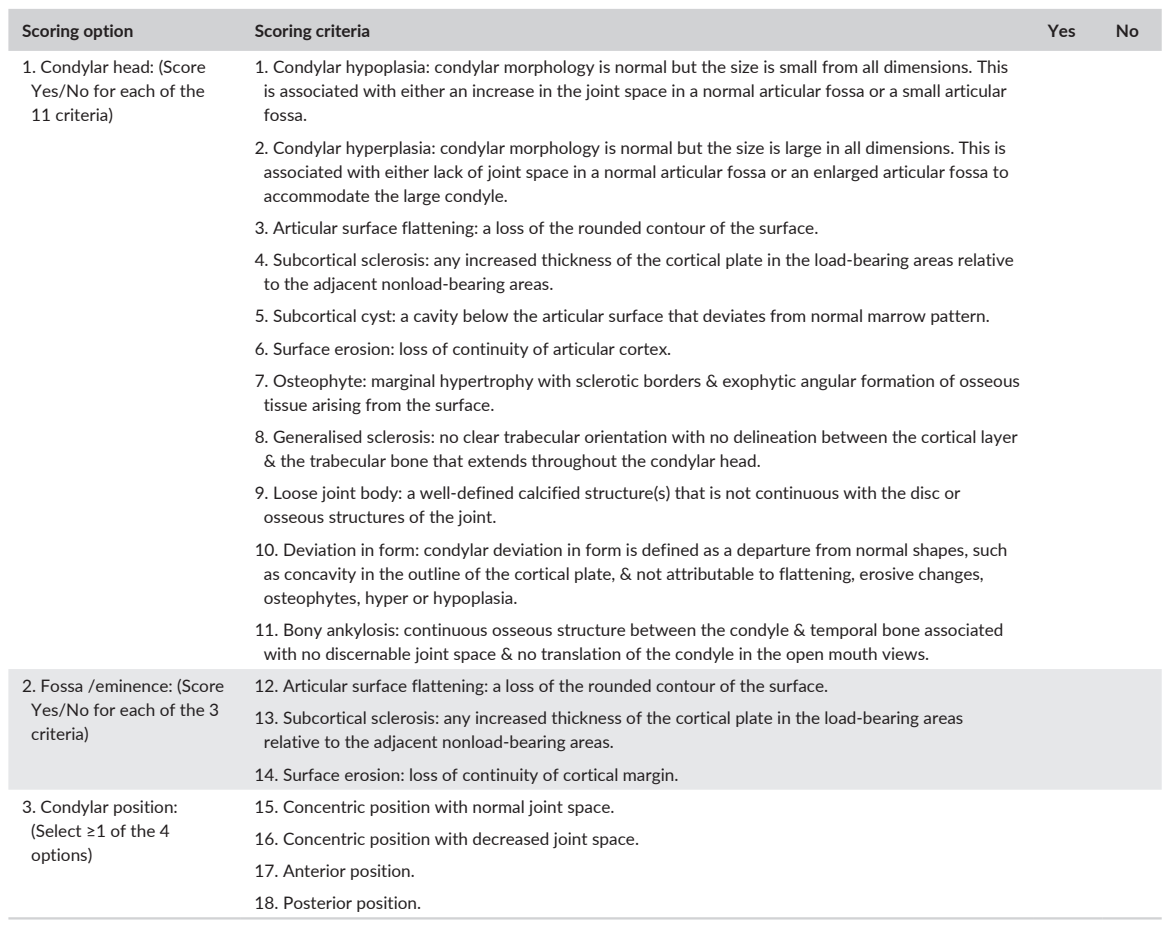

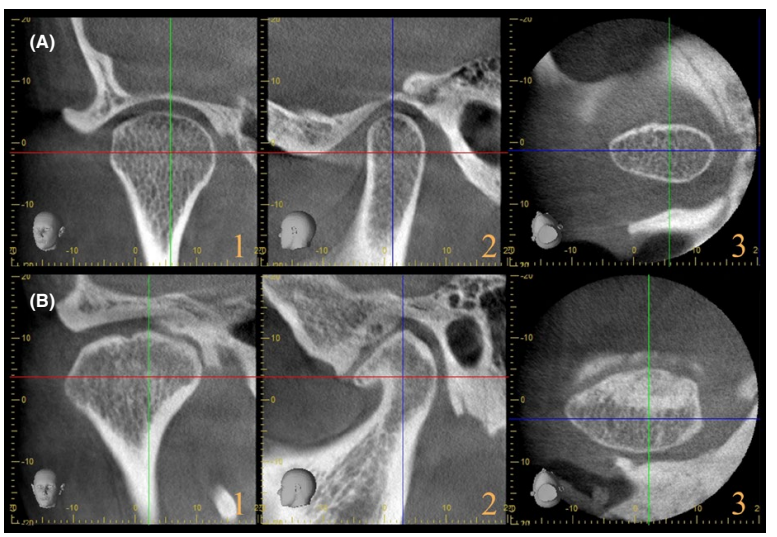

FIGURE 1 Examples of cone beam computed tomography images for 2 temporomandibular joint disorder cases. A, Case with no osseous changes, (B) Case with some osseous changes. (1) corrected coronal image, (2) corrected sagittal image and (3) axial image 
TABLE 2 Sensitivity and specificity of cone beam computed tomography protocol for assessing osseous changes of temporomandibular joint directly after the training and 2 mo later

\begin{tabular}{|c|c|c|c|c|}
\hline \multirow{2}{*}{$\begin{array}{l}\text { Radiographic diagnostic criteria /number of cases for each } \\
\text { criterion according to reference standard }\end{array}$} & \multicolumn{2}{|l|}{ Evaluation } & \multicolumn{2}{|c|}{ Re-evaluation } \\
\hline & Sensitivity & Specificity & Sensitivity & Specificity \\
\hline 1. Condylar hypoplasia /0 & - & 0.948 & - & 0.976 \\
\hline 2. Condylar hyperplasia /0 & - & 1.000 & - & 1.000 \\
\hline 3. Articular surface flattening /8 & 0.893 & 0.727 & 0.875 & 0.760 \\
\hline 4. Subcortical sclerosis /6 & 0.405 & 0.935 & 0.405 & 0.964 \\
\hline 5. Subcortical cyst /3 & 0.810 & 0.968 & 0.857 & 0.968 \\
\hline 6. Surface erosion /9 & 0.825 & 0.891 & 0.873 & 0.898 \\
\hline 7. Osteophyte /9 & 0.905 & 0.918 & 0.746 & 0.932 \\
\hline 8. Generalised sclerosis /0 & - & 0.976 & - & 0.900 \\
\hline 9. Loose joint body /1 & 1.000 & 0.980 & 1.000 & 1.000 \\
\hline 10. Deviation in form $/ 3$ & 0.333 & 0.868 & 0.238 & 0.931 \\
\hline 11. Bony ankylosis /0 & - & 1.000 & - & 0.995 \\
\hline 12. Articular surface flattening (fossa) $/ 5$ & 0.600 & 0.783 & 0.686 & 0.846 \\
\hline 13. Subcortical sclerosis (fossa) /6 & 0.381 & 0.857 & 0.476 & 0.929 \\
\hline 14. Surface erosion (fossa) /2 & 0.714 & 0.898 & 0.786 & 0.908 \\
\hline 15. Concentric position with normal joint space $/ 0$ & - & 0.481 & - & 0.469 \\
\hline 16. Concentric position with decreased joint space $/ 6$ & 0.476 & 0.976 & 0.595 & 0.970 \\
\hline 17. Anterior position $/ 9$ & 0.286 & 0.884 & 0.286 & 0.878 \\
\hline 18. Posterior position /3 & 0.714 & 0.905 & 0.667 & 0.905 \\
\hline
\end{tabular}

(-) Not relevant.

Assessment of the condylar head included noting gross hypoplasia or hyperplasia, flattening of the articular surface, any subcortical sclerosis or cysts, surface erosion, osteophytes, generalised or subcortical sclerosis, loose joint body, deviations in form and bony ankyloses. Assessment of the articular eminence included noting flattening of the articular eminence, subcortical sclerosis, and surface erosion. For condylar position, assessment included noting whether the joint condyle in anterior, posterior or centric position with normal or reduced joint space.

\section{4 | Statistical analysis}

Cone beam computed tomography protocol sensitivity and specificity were calculated using the 2-month follow-up readings, as well as observer performance and agreement. The latter was expressed as a percentage of correct answers in comparison with the reference standard, percentage of agreement between the observers and differences between the 2 readings. The analysis was made with the Statistical package for the social sciences (IBM SPSS, version 23 for Windows). The changes in overall response between the first and second evaluations were tested with a pairwise $t$ test.

\section{3 | RESULTS}

Table 2 presents the sensitivity and specificity of the CBCT protoco for assessing osseous changes of the TMJ. Sensitivity and specificity ranged from $75 \%$ to $100 \%$ except for some criteria. Average observer sensitivity was $65 \%$, whereas specificity was somewhat higher, on average $87 \%$. Criteria 1, 2, 8, 11 and 15 occurred in none of the cases. The mean observers' response correctly to the diagnostic criteria was $84 \%$ per observer while $73 \%$ was the mean agreement between them.

Figure 2 illustrates the differences in overall responses between the first and second evaluations. In general, observer responses improved at the 2-month follow-up compared with the first evaluation except for osteophyte and generalised sclerosis, although neither the training programme nor the evaluation programme contained a case of generalised sclerosis.

In Table 3, the result of the pairwise $t$ test on the change in overall response between the first and the second evaluations is presented.

\section{4 | DISCUSSION}

Optimal TMD treatment can be achieved through proper examination and diagnosis. According to Diagnostic criteria for TMD (DC/ TMD), ${ }^{15}$ a further investigation using $\mathrm{CBCT}$ is recommended only when standard treatment has failed. $\mathrm{A} \mathrm{CBCT}$ examination has recently been considered the reference standard examination as it is a dose and cost-effective alternative to $\mathrm{CT}$ for radiographic examination of osseous changes in the TMJ., ${ }^{6,16}$ As these examinations have become increasingly popular among non-specialists and the available expertise for interpretation currently fails to match demand, we explored 1 way 


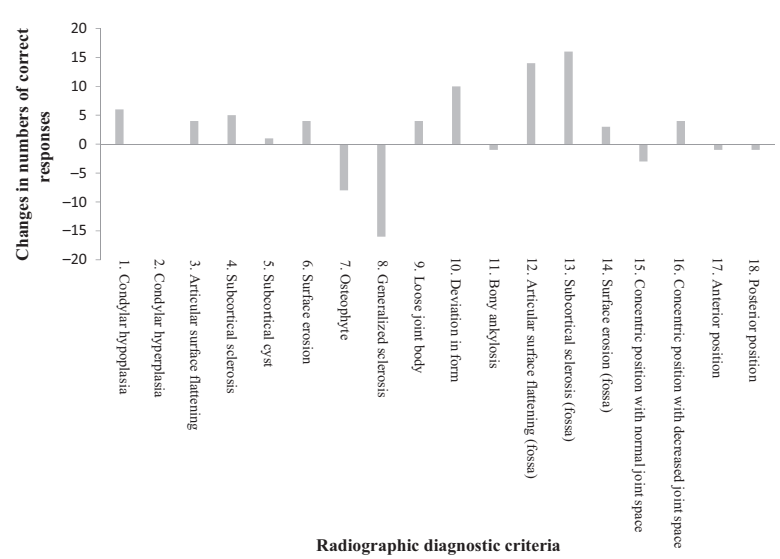

FIGURE 2 Changes in total number of correct responses between the 2 evaluations for the 7 general dental practitioners

to fill this gap. The present study found that Ahmed et al ${ }^{15}$ protocol can be used in a training programme to provide GDPs a simple way of learning about TMJ evaluation.

Currently, many countries have adopted the DC/TMD taxonomy as a classification scheme for standardising TMD clinical and radiographic examinations. ${ }^{17}$ According to DC/TMD, this study chose Ahmad et $\mathrm{al}^{15}$ criteria because they comprise a comprehensive, validated and reliable tool for assessing osseous changes of the TMJ radiographically. The training programme included only pre-defined $2 \mathrm{D}$ multiplane $\mathrm{CBCT}$ reconstructions to make the interpretation easier for the GDPs. The skills of setting the representative images, in 3 plans to display the region of interest appropriately, are usually difficult for non-radiologist.

To avoid possible misinterpretation, we adjusted all sagittal images in this study to be perpendicular to the condylar axis and corrected all coronal images to ensure they were parallel to the condylar axis.

Loose joint bodies derive from cartilaginous protuberances within the synovium that have detached. The high sensitivity of the loose joint body criterion (ie, the ability to detect the presence of osseous change on a CBCT image) might be explained by the ease of detecting radiopaque structures compared with radiolucent ones in a radiographic image and also, our choice of presenting good representative clear loose body joint cases. In contrast, condylar hyperplasia, hypoplasia, generalised sclerosis, bony ankyloses and concentric joint position with normal joint space did not occur in any case due to their rarity, which may explain why these criteria had the lowest sensitivity. Excluding these criteria would yield average correct observer readings of $70 \%-100 \%$ compared to the findings of the radiologists, which could be considered adequate. Among radiologist observers in a diagnostic performance comparison between CBCT and conventional $\mathrm{CT}$, Honda et $\mathrm{al}^{7}$ found a sensitivity of $80 \%$ for osseous changes. In contrast, however, Zain-Alabdeen et $\mathrm{al}^{8}$ reported lower sensitivity for detection of TMJ osseous changes in $\mathrm{CBCT}$ images.
In the present study, subcortical sclerosis, deviation in form and condylar position had lower sensitivities than all other criteria. In the literature, some reports found extensive variation in sensitivity for the condyle position criterion, although it has little clinical relevance and poor observer agreement. Subcortical sclerosis might be misinterpreted due to the lack of an exact definition of normal cortical thickness in the literature and deviation in form could be confused with other changes in shape that occur in TMD, such as osteophyte formation.

Specificity for all criteria (ie, the ability to detect the absence of osseous change on a CBCT image) was fairly high, which is similar to the findings of Honda et al and Zain-Alabdeen et $\mathrm{al}^{7,8}$ This means that GDPs were usually able to detect osseous changes and were even better in identifying TMJs without osseous changes that is, underestimating the number of joints with osseous changes.

All the observers in the present study performed well, despite minor experience with $\mathrm{CBCT}$ interpretation, so the rate of correct responses per observer (76\%-92\%) can be considered good. Additionally, the agreement between them was good, which was consistent with the 2 observers in the Zain-Alabdeen study ${ }^{8}$ who showed significant correlation. Marques et $\mathrm{al}^{18}$ found similar concordance upon the assessment of simulated condylar bony lesions using СВCT.

Protocol performance improved at the 2-month follow-up, compared with the results directly after the training programme. This suggests a knowledge gain and demonstrates the advantages of a training programme before image interpretation. Osteophyte detection was an exception and the performance on this criterion was lower than expected. Osteophytes are one of the hallmarks of degenerative changes in the joint; 1 study found commonly true positive findings for this criterion, particularly with increased size. ${ }^{19}$ Thus, the small osteophytes in all the study cases could explain the low observers' performance. The change in overall response between the first and second evaluations (Table 3 ) indicates significant differences between the 2 evaluations 
TABLE 3 The change in overall response between the first and second evaluations as given by a pairwise $t$ test

\begin{tabular}{|c|c|c|c|c|c|}
\hline \multirow{3}{*}{$\begin{array}{l}\text { Radiographic } \\
\text { diagnostic criteria }^{a}\end{array}$} & \multicolumn{5}{|c|}{ Test value $=0$} \\
\hline & \multirow[b]{2}{*}{$t$} & \multirow[b]{2}{*}{ Sig. (2-tailed) } & \multirow[b]{2}{*}{ Mean difference } & \multicolumn{2}{|c|}{$\begin{array}{l}95 \% \text { confidence } \\
\text { interval of the } \\
\text { difference }\end{array}$} \\
\hline & & & & Lower & Upper \\
\hline Condylar hypoplasia & 1.795 & 0.083 & 0.200 & -0.03 & 0.43 \\
\hline $\begin{array}{l}\text { Articular surface } \\
\text { flattening }\end{array}$ & 0.701 & 0.489 & 0.133 & -0.26 & 0.52 \\
\hline Subcortical sclerosis & -1.943 & 0.062 & -0.367 & -0.75 & 0.02 \\
\hline Subcortical cyst & 0.372 & 0.712 & 0.033 & -0.15 & 0.22 \\
\hline Surface erosion & 0.812 & 0.423 & 0.133 & -0.20 & 0.47 \\
\hline Osteophyte & -1.765 & 0.088 & -0.267 & -0.58 & 0.04 \\
\hline Generalised sclerosis & -3.764 & 0.001 & -0.533 & -0.82 & -0.24 \\
\hline Loose joint body & 2.112 & 0.043 & 0.133 & 0.00 & 0.26 \\
\hline Deviation in form & 2.276 & 0.030 & 0.333 & 0.03 & 0.63 \\
\hline Bony ankylosis & -1.000 & 0.326 & -0.033 & -0.10 & 0.03 \\
\hline $\begin{array}{l}\text { Articular surface } \\
\text { flattening (fossa) }\end{array}$ & 2.626 & 0.014 & 0.467 & 0.10 & 0.83 \\
\hline $\begin{array}{l}\text { Subcortical sclerosis } \\
\text { (fossa) }\end{array}$ & 3.247 & 0.003 & 0.533 & 0.20 & 0.87 \\
\hline Surface erosion (fossa) & 0.722 & 0.476 & 0.100 & -0.18 & 0.38 \\
\hline $\begin{array}{l}\text { Concentric position } \\
\text { with normal joint } \\
\text { space }\end{array}$ & -0.385 & 0.703 & -0.100 & -0.63 & 0.43 \\
\hline $\begin{array}{l}\text { Concentric position } \\
\text { with decreased joint } \\
\text { space }\end{array}$ & 0.941 & 0.345 & 0.133 & -0.16 & 0.42 \\
\hline Anterior position & -0.143 & 0.887 & -0.033 & -0.51 & 0.44 \\
\hline Posterior position & -0.273 & 0.787 & -0.033 & -0.28 & 0.22 \\
\hline
\end{tabular}

${ }^{a}$ There was no change in overall response between the 2 evaluations for condylar hyperplasia. for generalised sclerosis, loose joint body, deviation in form, articular surface flattening (fossa) and subcortical sclerosis (fossa).

The results of the current study should be interpreted with caution. One limitation is that we selected representative images for each of the chosen cases of TMD. We did not use the whole CBCT volume and this could influence the ability of the observers to examine cases. Finally, the training programme did not cover all of the osseous changes described in the protocol because some changes seldom occur.

\section{5 | CONCLUSION}

We believe that the present educational programme is a helpful training material on recognising possible osseous changes of TMJ and it can be used as a part of a training programme for GDP and for specialist candidates.

\section{CONFLICT OF INTEREST}

The authors declare no conflict of interests.

\section{ACKNOWLEDGMENTS}

The authors thank Arne Petersson, Lars-Göran Hansson and the GDPs for participating in this study, and special thanks to King Abdulaziz University for funding this study.

\section{ORCID}

D. Iskanderani (iD http://orcid.org/0000-0001-5593-0553

\section{REFERENCES}

1. Okeson J. Orofacial Pain. Carol Stream, IL: Quintessence Publishing; 1996.

2. Schmitter $M$, Kress B, Rammelsberg P. Temporomandibular joint pathosis in patients with myofascial pain: a comparative analysis of magnetic resonance imaging and a clinical examination based on a specific set of criteria. Oral Surg Oral Med Oral Pathol Oral Radio Endod. 2004;97:318-324.

3. White SC, Pullinger AG. Impact of TMJ radiographs on clinician decision making. Oral Surg Oral Med Oral Pathol Oral Radiol Endod. 1995;79:375-381. 
4. Hashimoto K, Arai Y, Iwai K, Araki M, Kawashima S, Terakado M. A comparison of a new limited cone beam computed tomography machine for dental use with a multidetector row helical CT machine. Oral Surg Oral Med Oral Pathol Oral Radiol Endod. 2003;95: 371-377.

5. Hashimoto K, Kawashima S, Kameoka S, et al. Comparison of image validity between cone beam computed tomography for dental use and multidetector row helical computed tomography. Dentomaxillofac Radiol. 2007;36:465-471.

6. Tsiklakis K, Syriopoulos K, Stamatakis HC. Radiographic examination of the temporomandibular joint using cone beam computed tomography. Dentomaxillofac Radiol. 2004;33:196-201.

7. Honda K, Larheim TA, Maruhashi K, Matsumoto K, Iwai K. Osseous abnormalities of the mandibular condyle: diagnostic reliability of cone beam computed tomography compared with helical computed tomography based on an autopsy material. Dentomaxillofac Radiol. 2006:35:152-157.

8. Zain-Alabdeen EH, Alsadhan RI. A comparative study of accuracy of detection of surface osseous changes in the temporomandibular joint using multidetector CT and cone beam CT. Dentomaxillofac Radiol. 2012;41:185-191.

9. Palconet G, Ludlow JB, Tyndall DA, Lim PF. Correlating cone beam $\mathrm{CT}$ results with temporomandibular joint pain of osteoarthritic origin Dentomaxillofac Radiol. 2012;41:126-130.

10. Horner K, Islam M, Flygare L, Tsiklakis K, Whaites E. Basic principles for use of dental cone beam computed tomography: consensus guidelines of the European Academy of Dental and Maxillofacial Radiology. Dentomaxillofac Radiol. 2009;38:187-195.

11. SEDENTEXCT Guidelines. http://www.sedentexct.eu/training/index. html. Accessed December 19, 2015.

12. Brown J, Jacobs $R$, Levring Jäghagen $E$, et al. Basic training require ments for the use of dental CBCT by dentists: a position paper prepared by the European Academy of DentoMaxilloFacial Radiology. Dentomaxillofac Radiol. 2014;43:20130291.
13. Gava MM. What the general dental practitioner should know about cone beam computed tomograph technology. OHDMBSC. 2009;8:14-21.

14. Al-Rawi WT, Jacobs R, Hassan BA, Sanderink G, Scarfe WC. Evaluation of web-based instruction for anatomical interpretation in maxillofacial cone beam computed tomography. Dentomaxillofac Radiol. 2007;36:459-464.

15. Ahmad M, Hollender L, Anderson Q, et al. Research diagnostic criteria for temporomandibular disorders (RDC/TMD): development of image analysis criteria and examiner reliability for image analysis. Oral Surg Oral Med Oral Pathol Oral Radiol Endod. 2009;107:844-860.

16. Barghan S, Tetradis S, Mallya S. Application of cone beam computed tomography for assessment of the temporomandibular joints. Aust Dent J. 2012;57(Suppl 1):109-118.

17. Schiffman E, Ohrbach R, Truelove E, et al. Diagnostic criteria for temporomandibular disorders (DC/TMD) for clinical and research applications: recommendations of the International RDC/TMD Consortium Network and Orofacial Pain Special Interest Group. J Oral Facial Pain Headache. 2014;28:6-27.

18. Marques AP, Perrella A, Arita ES, Pereira MF, Cavalcanti MG. Assessment of simulated mandibular condyle bone lesions by cone beam computed tomography. Braz Oral Res. 2010;24:467-474.

19. Cholitgul W, Petersson A, Rohlin M, Tanimoto K, Akerman S. Diagnostic outcome and observer performance in sagittal tomography of the temporomandibular joint. Dentomaxillofac Radiol. 1990;19:1-6.

How to cite this article: Iskanderani $D$, Alstergren $P$, Hellén-Halme K. Assessment of a training programme on detection of temporomandibular joint osseous changes applying pre-defined 2D multiplane cone beam computed tomography reconstructions. J Oral Rehabil. 2018;45:282-288. https://doi. org/10.1111/joor.12610 

II 



\title{
Web-based educational programme for temporomandibular joint assessment with cone-beam computed tomography
}

\author{
Durer Iskanderani $^{1,2}$ (D) | Per Alstergren ${ }^{3}$ | Ewa Carin Ekberg ${ }^{3}$ | Xie-Qi Shi ${ }^{1,4}$ | \\ Kristina Hellén-Halme ${ }^{1}$
}

${ }^{1}$ Department of Oral and Maxillofacial Radiology, Faculty of Odontology, Malmö University, Malmö, Sweden

${ }^{2}$ Department of Oral and Maxillofacial Radiology, Faculty of Dentistry, King Abdulaziz University, Jeddah, Saudi Arabia

${ }^{3}$ Department of Orofacial Pain and Jaw Function, Faculty of Odontology, Malmö University, Malmö, Sweden

${ }^{4}$ Division of Oral Maxillofacial Radiology, Department of Clinical Dentistry, Faculty of Medicine, University of Bergen, Bergen, Norway

\section{Correspondence}

Durer Iskanderani, Department of Oral and Maxillofacial Radiology, Faculty of Odontology, Malmö University, Malmö SE205 06, Sweden.

Email: durer.iskanderani@mau.se

Funding information

King Abdulaziz University

\begin{abstract}
Objectives: To develop and evaluate an educational web-based programme for temporomandibular joint (TMJ) assessment using cone-beam computed tomography (CBCT).

Methods: A web-based educational programme was designed for TMJ assessment using CBCT images. Fifteen CBCT cases of the TMJ (three-dimensional reconstructed volumes) and an assessment module based on image analysis criteria in the Diagnostic Criteria for Temporomandibular Disorders (DC/TMD) were added to the programme. Thirty-six undergraduate dental students were invited to test the programme by assessing all cases twice: before and after 2 months of training. Participants submitted written subjective evaluations of the programme.

Results: The web-based programme can be accessed on Malmö University webpage (www.cbct-tmj.mau.se). Despite limited CBCT learning in their undergraduate training and not using the programme in the 2-month interval, the students were able to correctly diagnose $80 \%$ of the cases at the second assessment. Their diagnoses, however, did not differ significantly from the first assessment. Overall, the students were satisfied with the programme and considered it user-friendly.

Conclusion: The web-based educational programme that was developed in the present study and tested by dental students could be a useful educational tool for TMJ assessment using CBCT.

KEYWORDS

cone-beam $\mathrm{CT}$, education, temporomandibular joint, training programme
\end{abstract}

\section{1 | INTRODUCTION}

Cone-beam computed tomography (CBCT) examination of the temporomandibular joint (TMJ) has become a recognised tool in case of suspicion for the presence of osseous changes, after a thorough history and clinical examination of the patient. $\mathrm{CBCT}$ images provide high spatial resolution in three-dimensional (3D) images, which is desirable in diagnostics and treatment of temporomandibular disorder
(TMD). Often, its diagnostic accuracy and reliability are better than that of two-dimensional (2D) images. ${ }^{1} \mathrm{CBCT}$ provides comparable diagnostic accuracy with a relatively lower radiation dose and cost compared with medical computed tomography $(\mathrm{CT})^{2-4}$ and, at present, is considered the technique of choice for evaluating osseous changes of the TMJ. ${ }^{5}$

Researchers in the International Network for Orofacial Pain and Related Disorders Methodology (INfORM; formerly, the International

The peer review history for this article is available at https://publons.com/publon/10.1111/joor.13065. 
RDC/TMD Consortium Network) developed the Diagnostic Criteria for TMDs (DC/TMD) in order to standardise the diagnosis of TMD globally. ${ }^{6}$ These criteria have been widely accepted and used as a valid diagnostic tool for most common conditions of oro-facial pain and jaw dysfunction. In clinical and research settings, the DC/TMD allows for identification of patients with a range of simple to complex TMD presentations. ${ }^{7}$ Along with DC/TMD, Ahmad et al ${ }^{8}$ reported comprehensive and reliable image analysis criteria for assessing the TMJ, which describe the soft and hard tissue changes that may occur in the TMJ.

Rapid advances in digital and web technologies, and their use in education, have focused attention on alternative methods of education. Thus, a web-based approach has become a feasible and practical form of learning. Besides allowing choice of time and place of learning, web-based approach provides learners with an interactive self-learning tool.

The application of CBCT imaging in dental practice is increasing; consequently, it raised a demand for more education about this technique. Studies on pre- and/or post-doctoral dental education using $\mathrm{CBCT}$ imaging reported some gaps; one of them was image interpretation. ${ }^{9,10}$ Thus, a web-based learning programme on CBCT interpretation might be an efficient and practical way to increase the knowledge and adjunct the educational process. Several studies have emphasised the advantages of web-based learning in dental education. ${ }^{11-13}$ Howerton et al ${ }^{14}$ reported similar learning outcomes between computer-assisted and conventional learning methods with the computer-assisted method being preferred by students.

Various CBCT online training programmes are available to support dental education in radiology, and they are useful and show high efficacy in delivering knowledge. ${ }^{15-18}$ Our research team recently published a training programme on the detection of TMJ osseous changes using predefined $2 \mathrm{D}$ multiplane $\mathrm{CBCT}$ reconstructions. ${ }^{18}$ Thus, the purpose of this study was to develop and evaluate the usefulness of a web-based educational programme for TMJ assessment using CBCT.

\section{MATERIALS AND METHODS}

\section{1 | Radiographic examination}

Fifteen CBCT cases of the TMJ were retrieved, to meet the scope of the study, from the Faculty of Odontology database at Malmö University, Malmö, Sweden. The CBCT volumes were acquired with a Veraviewepocs 3D F40 scanner (J. Morita Corp., Kyoto, Japan) using the manufacturer's recommended scanning protocol $(90 \mathrm{kV}, 5 \mathrm{~mA}$, and $9.4 \mathrm{~s})$. All images were exposed with a $40 \times 40 \mathrm{~mm}$ field of view, $180^{\circ}$ rotation, and reconstructed with a voxel size of $0.125 \mathrm{~mm}, 1 \mathrm{~mm}$ slice thickness and $1 \mathrm{~mm}$ slice interval. For each case, the three orthogonal volumes (axial, coronal and sagittal) were exported separately and then imported to the Synedra image viewer (Synedra Information Technologies, Innsbruck, Austria). In the viewer, all cases were anonymised and the image stacks were converted to the JPEG file format in order to be compatible with web browsers.

\section{2 | Study participants}

Thirty-six undergraduate dental students in their final year at the Faculty of Odontology, Malmö University were recruited to this study. None of the students had previous training in interpretation of CBCT images of the TMJ.

\section{3 | Radiographic assessment}

The assessment module for the TMJ osseous changes was structured according to DC/TMD image analysis criteria. ${ }^{8}$ The module describes the criteria in the following sequence: changes in the condylar head, the articular eminence and condylar position. Assessment of the condylar head involves observing gross hypoplasia or hyperplasia, flattening of the articular surface, any subcortical sclerosis or cysts, surface erosion, osteophytes, generalised or subcortical sclerosis, loose joint body, deviations in form and bony ankyloses. Assessment of the articular eminence involves observing flattening of the articular eminence, subcortical sclerosis and surface erosion. For the condylar position, assessment involves determining whether the joint condyle is in an anterior, posterior or centric position with normal or reduced joint space. One of three diagnoses is then set: no degenerative joint disease, indeterminate for degenerative joint disease or degenerative joint disease (Table 1).

\section{4 | Programme development}

The web-based programme was designed with the help of professional web designers using three open-source web frameworks: Laravel, React/Redux and d3. The bulk of the programme was built using the PHP-based Laravel framework to implement back-end functionality (eg user accounts, application programming interface endpoints and database and content management) as well as frontend user and admin HTML pages.

The case assessment pages were developed using JavaScript libraries: for the user interface-React, as the web framework; and for state management-Redux. The pages were built as a single-page JavaScript-driven application with no page reloads between cases, rather than as a series of separate HTML pages. Each case is presented on a separate screen. The CBCT examination screen (Figure 1A) presents a series of high-resolution JPEG images for each of the three volumes, similar to what would be viewed in the clinical situation, while keeping bandwidth requirements and loading times low. The assessment module allows only dichotomous responses, presence/absence for each criterion. After assessing all criteria, users must set a diagnosis to proceed to next case. User instructions and list of the assessment criteria can be accessed via a button at the bottom of each case screen and when the curser points to a criterion or diagnosis, an explanation appears in a window to the left (Figure 1A). Reference findings were compiled through consensus by a team of three senior specialists in oral and maxillofacial radiology, 
TABLE 1 The criteria and diagnoses used in the webbased programme for assessing osseous changes of the temporomandibular joint on cone-beam computed tomography images; structured according to Ahmad et al ${ }^{8}$

Criteria/number of cases for each criterion according to the reference standard

\section{Condylar head}

1. Condylar hypoplasia: condylar morphology is normal but the size is small from all dimensions. This is associated with either an increase in the joint space in a normal articular fossa or a small articular fossa/0

2. Condylar hyperplasia: condylar morphology is normal but the size is large in all dimensions. This is associated with either lack of joint space in a normal articular fossa or an enlarged articular fossa to accommodate the large condyle/ 0 .

3. Articular surface flattening: a loss of the rounded contour of the surface $/ 6$

4. Subcortical sclerosis: any increased thickness of the cortical plate in the load-bearing areas relative to the adjacent non-load-bearing areas $/ 4$.

5. Subcortical cyst: a cavity below the articular surface that deviates from normal marrow pattern/6.

6. Surface erosion: loss of continuity of articular cortex/10.

7. Osteophyte: marginal hypertrophy with sclerotic borders and exophytic angular formation of osseous tissue arising from the surface/6.

8. Generalised sclerosis: no clear trabecular orientation with no delineation between the cortical layer and the trabecular bone that extends throughout the condylar head/3.

9. Loose joint body: a well-defined calcified structure(s) that is not continuous with the disc or osseous structures of the joint/1.

10. Deviation in form: condylar deviation in form is defined as a departure from normal shapes, such as concavity in the outline of the cortical plate, and not attributable to flattening, erosive changes, osteophytes, hyper or hypoplasia/3.

11. Bony ankylosis: continuous osseous structure between the condyle and temporal bone associated with no discernible joint space and no translation of the condyle in the open mouth views $/ 0$. Fossa/eminence

12. Articular surface flattening: a loss of the rounded contour of the surface/7.

13. Subcortical sclerosis: any increased thickness of the cortical plate in the load-bearing areas relative to the adjacent non-loadbearing areas/ 7 .

14. Surface erosion: loss of continuity of cortical margin/8.

Condylar position

15. Concentric position with normal joint space/7.

16. Concentric position with decreased joint space/4

17. Anterior position $/ 3$

18. Posterior position/4

Diagnosis/number of cases for each diagnosis according to the reference standard

A No degenerative joint disease/3

i. Normal relative size of the condylar head; and

ii. No subcortical sclerosis or articular surface flattening; and

iii. No deformation due to subcortical cyst, surface erosion,

osteophyte, generalised sclerosis or loose calcified bodies.

$B$ Indeterminate for degenerative joint disease/1
TABLE 1 (Continued)

i. Normal relative size of the condylar head; and

ii. Subcortical sclerosis with/without articular surface flattening; or

iii. Articular surface flattening with/without subcortical sclerosis;

and

iv. No deformation due to subcortical cyst, surface erosion, osteophyte, generalised sclerosis or loose calcified bodies.

\section{Degenerative joint disease $/ 11$}

i. Deformation due to subcortical cyst, surface erosion, osteophyte, generalised sclerosis or loose calcified bodies.

each with more than 10 years of experience in evaluating TMJs on CBCT images. A final programme feature used JavaScript library $d 3$ to build an interactive matrix, which was based on all responses to the cases compared with reference findings. It describes user performance at the end of all cases for user learning (Figure 1B).

The administration page was designed to give the authors control over the programme setup with safeguards for guaranteeing data integrity. The programme was divided into four phases, which the authors unlocked successively via the administration page; thus, limitations were placed on the users, to mitigate the risk of manual mistakes.

\subsection{1 | Phase I: Preparation}

An online account for each study participant was created, and radiographic images for each case were uploaded to the programme. Each time a user started the programme, and the cases were presented in a new, randomised order with all personal, clinical and radiographrelated data anonymised. None of the cases could be related to an underlying disease or malignancy, and they covered a wide range of osseous changes in the TMJ; some healthy cases were also included. The programme was designed to lock all website elements after a user started the programme to guarantee data consistency.

\subsection{2 | Phase II: The first assessment and training}

One author (DI) introduced participants to the web-based programme in an oral presentation using case examples and illustrations to describe the design of the programme, how to use it, and the criteria for diagnosing osseous changes in the TMJ. Participants were then assigned login credentials and asked to make a first assessment of the programme. All participants evaluated the cases independently on their own computers. Case assessment was not time limited, and responses were saved in real time on a remote server, which let the participants exit the programme and resume at any time.

When participants had finished their assessment of the cases, they were shown the interactive results matrix (Figure 1B). By clicking on a response in the matrix, they could see the case and compare their response with the reference findings. In the 2 months following this first assessment, participants were allowed to use the programme to train diagnoses of osseous changes in the TMJ whenever they chose. 


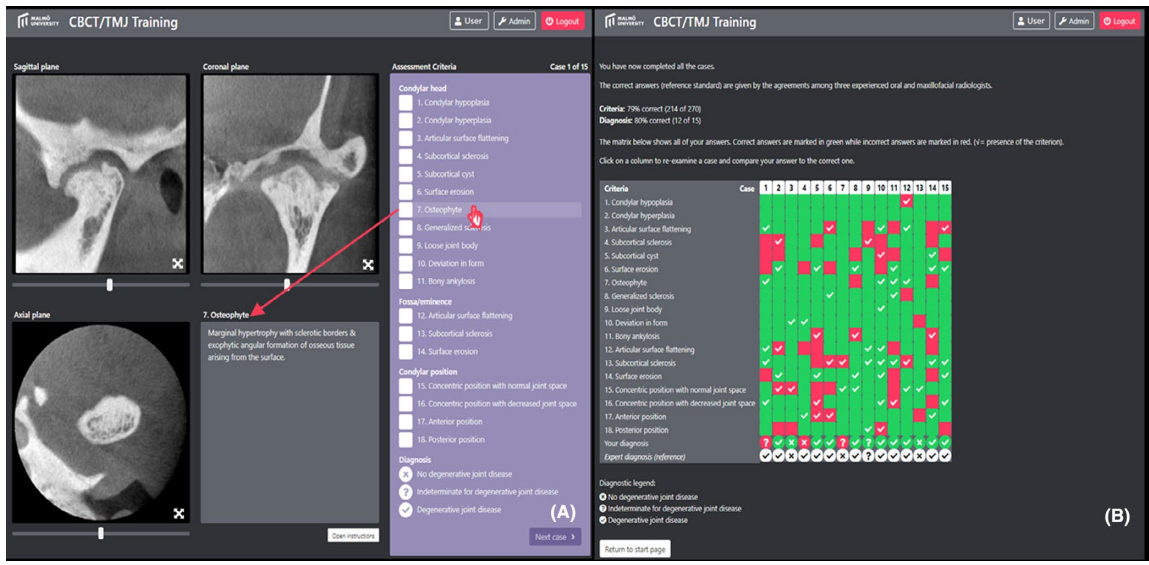

FIGURE 1 A screenshot of the web-based programme for temporomandibular joint interpretation on cone-beam computed tomography images (CBCT). A, case assessment page showing the reconstructed 3D CBCT images (sagittal, coronal, and axial) and the list of assessment criteria; when the cursor points to a criterion or a diagnosis, an explanation appears in a window to the left. B, User result matrix that compares user and reference responses for all cases

\subsection{3 | Phase III: The second assessment}

At the 2-month follow-up, participants were asked to make a second assessment of all cases in the programme.

\subsection{4 | Phase IV: Going public}

Following an acceptable user assessment, we opened the programme to the public. In this phase, users did not need to sign up for an account.

\section{5 | Programme evaluation}

At the end of Phase III, the participants were asked to subjectively evaluate their experience of using the programme on a form. The questions concerned accessibility, design, radiographic image quality, the results matrix, knowledge gained and the web-based learning approach as a teaching method (Table 2). Responses were made using a 5-point Likert scale: 1 = extremely satisfied, 2 = moderately satisfied, $3=$ neutral, $4=$ moderately unsatisfied and $5=$ extremely unsatisfied.

\section{6 | Statistical analysis}

The web-based programme stored the results of each participant on a Microsoft Excel 2016 spreadsheet. The Statistical Package for the Social Sciences (IBM SPSS, version 23 for Windows) was used to analyse participants' diagnostic performance and subjective evaluation of the programme. The changes in the diagnosis responses between the first and second assessments were analysed using the Sign test ${ }^{19}$ with the level of significance set to 0.05 .

\section{3 | RESULTS}

A web-based educational programme for the assessment of TMJ osseous changes in CBCT images, based on the DC/TMD image analysis criteria, ${ }^{8}$ was developed and can be accessed on Malmö university webpage (www.cbct-tmj.mau.se). The programme consists of $15 \mathrm{TMJ}$ cases presented as 3D reconstructed $\mathrm{CBCT}$ volumes, along with the assessment module (Figure 1A).

Figure 2 presents the overall percentage of participants' correct diagnosis response for all cases, at the first and second assessments. Figure 3 shows the changes in overall percentage of correct diagnosis responses between the two assessments, for all participants and cases. In most cases, the diagnostic performance improved; however, the differences in correct diagnoses between the two assessments (76\% at assessment 1 and $80 \%$ at assessment 2 ) were not significant $(P=0.1-1.0)$.

Table 3 presents participants' evaluation of the web-based programme. In general, more than $90 \%$ of the participants were moderately or extremely satisfied and thought that the programme was easy to access and had a user-friendly design, while more than $65 \%$ were moderately or extremely satisfied with the radiographic image quality and results matrix. Seventy-six per cent of the participants were moderately or extremely satisfied with the knowledge gained after using the programme and with its value as a learning tool. 
TABLE 2 Form for evaluating how participants experienced the web-based programme

\begin{tabular}{|c|c|c|c|c|c|}
\hline & \multicolumn{5}{|c|}{ Level of satisfaction } \\
\hline & $\begin{array}{l}\text { Extremely } \\
\text { satisfied }\end{array}$ & $\begin{array}{l}\text { Moderately } \\
\text { satisfied }\end{array}$ & Neutral & $\begin{array}{l}\text { Moderately } \\
\text { unsatisfied }\end{array}$ & $\begin{array}{l}\text { Extremely } \\
\text { unsatisfied }\end{array}$ \\
\hline $\begin{array}{l}\text { 1. The programme contents were easy to } \\
\text { access. }\end{array}$ & $\circ$ & $\circ$ & $\circ$ & $\circ$ & $\circ$ \\
\hline $\begin{array}{l}\text { 2. The programme's design was well- } \\
\text { organised and user-friendly. }\end{array}$ & $\circ$ & $\circ$ & ० & $\circ$ & $\circ$ \\
\hline $\begin{array}{l}\text { 3. Image resolution was high and the } \\
\text { anatomical landmarks were clearly visible. }\end{array}$ & $\circ$ & $\circ$ & $\circ$ & $\circ$ & $\circ$ \\
\hline $\begin{array}{l}\text { 4. The results matrix was understandable and } \\
\text { made it easy to track the answers. }\end{array}$ & $\circ$ & $\circ$ & 0 & $\circ$ & $\circ$ \\
\hline $\begin{array}{l}\text { 5. The programme challenged your previous } \\
\text { knowledge in such a way that your } \\
\text { understanding was widened. }\end{array}$ & $\circ$ & $\circ$ & $\circ$ & ० & $\circ$ \\
\hline $\begin{array}{l}\text { 6. The web-based learning approach makes } \\
\text { learning faster, easier and interactive } \\
\text { (accessible anytime, anywhere). }\end{array}$ & $\circ$ & $\circ$ & $\circ$ & $\circ$ & $\circ$ \\
\hline
\end{tabular}

FIGURE 2 The overall percentage of participants' correct diagnosis response, for all cases at each assessment

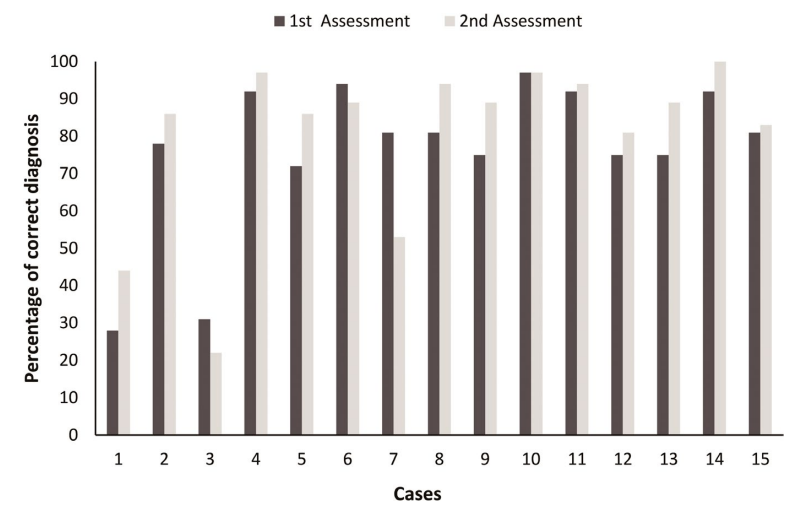

FIGURE 3 The changes in overall percentage of correct diagnosis responses between the two assessments for all participants

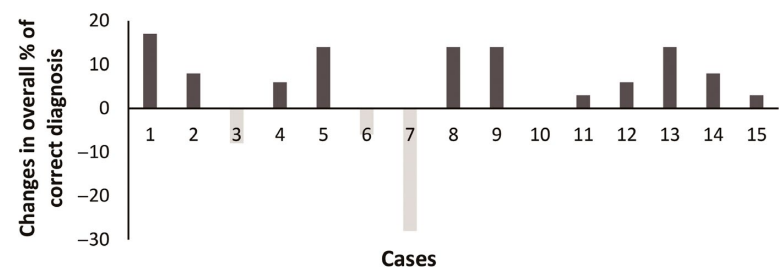

\section{4 | DISCUSSION}

When investigating volumetric $\mathrm{CBCT}$ images, the observer can scrol through the entire volume in many viewing plans in order to visualise the image data set and answer a clinical question. Thus, the images provide extensive and detailed information. Therefore, a thorough knowledge of $\mathrm{CBCT}$ anatomy, anatomic variations, tissue abnormalities and pathologies is prerequisites to $\mathrm{CBCT}$ image interpretation, which is not commonly part of general dental education.

Recently, we developed a training programme that describes TMJ osseous changes but incorporated predefined 2D multiplane CBCT reconstructions. General dental practitioners who participated 
TABLE 3 Students subjective evaluation of the web-based programme for diagnosing osseous changes of the temporomandibular joint on cone-beam computed tomography images ( $n=36$ undergraduate dental students, after the second assessment)

\begin{tabular}{|c|c|c|c|c|c|}
\hline & $\begin{array}{l}\text { Extremely satisfied } \\
\text { (\%) }\end{array}$ & $\begin{array}{l}\text { Moderately satisfied } \\
\text { (\%) }\end{array}$ & Neutral (\%) & $\begin{array}{l}\text { Moderately unsatisfied } \\
\text { (\%) }\end{array}$ & $\begin{array}{l}\text { Extremely } \\
\text { unsatisfied (\%) }\end{array}$ \\
\hline Accessibility & 53 & 45 & 2 & - & - \\
\hline Design & 67 & 26 & 7 & - & - \\
\hline Radiographic image quality & 45 & 29 & 24 & 2 & - \\
\hline Results matrix & 26 & 38 & 19 & 10 & 7 \\
\hline Knowledge gained & 33 & 43 & 17 & 7 & - \\
\hline $\begin{array}{l}\text { Web-based learning } \\
\text { approach }\end{array}$ & 40 & 36 & 17 & 5 & 2 \\
\hline
\end{tabular}

in the previous programme reported the programme to be helpful training material. ${ }^{18}$ Its limitation, however, was the use of predefined $2 \mathrm{D}$ images instead of the entire CBCT volume. Thus, the present project was to resolve this limitation by developing a pedagogical web-based programme for $\mathrm{CBCT}$ interpretation of the TMJ using $3 \mathrm{D}$ reconstructed images that mimic the clinical situation with the ability of the observer to scroll though the volume in 3D plans. This interpretation was based on the recommendation of INfORM ${ }^{6}$; that (the DC/TMD) has been recognised as a standardised and valid diagnostic tool for TMDs. The goal of the present study was to develop a comprehensive, self-learning and interactive platform for the interpretation of degenerative joint disease using the СВCT examination as an educational tool. It was proposed to be useful to anyone interested in the subject such as a general clinician, under or postgraduate dental student, or specialist for training, calibrating an observer and/or increasing knowledge about the subject.

The web-based training approach provides ease and flexibility of learning so that users are unlimited in time and place of learning. The presented programme can be used on a range of devices, including laptops, iPads/tablets and smartphones. Also, it has the advantages of knowledge delivery in multiple formats (eg 3D images and interactive assessment modules), and storage of results between learning sessions to enable tracking of performance (ie the user can pause the programme and continue at any time). Thus, this programme could, in a long run, reduce effort, cost and time of the teaching process. Mitov et al ${ }^{13}$ recommended the online dental learning approach as a valuable tool for self-learning. The Clark ${ }^{12}$ study suggested that incorporating the web-based learning approach into dental education would be beneficial.

To develop a learning tool, we believe that using recognised criteria, based on evidence from well-conducted researches, is beneficial. Since the criteria are valid and reliable tool and are accepted internationally, therefore, it could help to provide knowledge in a standardised way. Hellen-Halme et $\mathrm{al}^{20}$ and our research team in the previous study ${ }^{18}$ reported the usefulness of using Ahmad et al ${ }^{8}$ criteria. Both teams assumed that it could increase the diagnostic outcome among observers for magnetic resonance imaging (MRI) and $\mathrm{CBCT}$ evaluations of the TMJ, respectively. Our results showed good participant performance in the assessment module. One criterion, 'deviation in form', was poorly understood and affected the diagnostic response in case \# 7 (Figure 3). Perhaps, it was being confused with other condylar changes in form. A similar situation occurred in the previous $2 \mathrm{D}$ training programme. ${ }^{18}$

All students in their final year were invited; however, the response rate was $61 \%$, with only 5 of 36 participants have done some training. The assessment session was designed to be one hour at most, and therefore, the number of cases was limited to 15 . We believe that evaluating radiographs for more than an hour can be tiring, and thereby, the results would be affected. Prior participants' knowledge of CBCT interpretation was limited as little involvement of $\mathrm{CBCT}$ imaging during undergraduate dental education. Thus, a thorough oral presentation was delivered to them. However, when the programme opened to the public, user instructions and a description of the diagnostic criteria were provided. The participants were correct in their diagnosis by $76 \%$, which is a good performance at the first assessment and could indicate that the programme is understandable. A similar performance was observed in the second assessment by $80 \%$ correct diagnosis, which could suggest that they reasonably grasp the presented knowledge without a noticeable improvement; therefore, they may need more practice. Hellen-Halme et $\mathrm{al}^{20}$ developed a comparable web-based programme for examining soft tissue changes in the TMJ using MRI and following Ahmad et $\mathrm{al}^{8}$ criteria.

During a case assessment, the participant can enlarge the 3D reconstructed volumes by clicking on the lower right corner of the selected plane as well as scrolling through the volume (Figure 1). We think that our programme presents cases in a well-organised manner with an interactive portion and was easy and time-efficient. These reasons could explain the overall encouraging attitude of the participants towards the programme. They appreciated the web-based learning method and were satisfied with the contents of the programme. The participants were, however, less satisfied with the results matrix. A web-based programme for anatomical interpretation in maxillofacial CBCT images showed comparable results for the participants' performance after using the programme with a highly positive perception. ${ }^{16,17}$

A limitation of this study was that the authors have developed and evaluated the programme according to their knowledge of TMD. In addition, not all DC/TMD image analysis criteria were represented in the programme's cases. However, the satisfaction of most of the participants can be a strength. 
The present study, in line with several previous studies, points out numerous benefits of the web-based learning approach. In addition, it shows that similar educational materials could be advantageous for the education of radiographic interpretation.

\section{5 | CONCLUSION}

The web-based educational programme that was developed in the present study and tested by dental students could be a useful educational tool for TMJ assessment using CBCT.

\section{ACKNOWLEDGMENTS}

The authors thank Daniel Dunér and Jonatan Alvarsson for their work in developing the web-based programme. This study was financially supported by King Abdulaziz University.

\section{CONFLICTS OF INTEREST}

The authors declare no conflicts of interest.

ORCID

Durer Iskanderani (D) https://orcid.org/0000-0001-5593-0553

\section{REFERENCES}

1. Honey OB, Scarfe WC, Hilgers MJ, et al. Accuracy of cone-beam computed tomography imaging of the temporomandibular joint: comparisons with panoramic radiology and linear tomography. Am Orthod Dentofacial Orthop. 2007:132:429-438.

2. Honda K, Larheim TA, Maruhashi K, Matsumoto K, Iwai K. Osseous abnormalities of the mandibular condyle: diagnostic reliability of cone beam computed tomography compared with helical computed tomography based on an autopsy material. Dentomaxillofac Radiol. 2006;35:152-157.

3. Sirin Y, Guven K, Horasan S, Sencan S. Diagnostic accuracy of cone beam computed tomography and conventional multislice spiral tomography in sheep mandibular condyle fractures. Dentomaxillofac Radiol. 2010;39:336-342.

4. Kadesjö N, Benchimol D, Falahat B, Näsström K, Shi XQ. Evaluation of the effective dose of cone beam computed tomography and multi-slice computed tomography for temporomandibular joint examinations at optimized exposure levels. Dentomaxillofac Radiol. 2015:44:20150041.

5. Tsiklakis K, Syriopoulos K, Stamatakis HC. Radiographic examination of the temporomandibular joint using cone beam computed tomography. Dentomaxillofac Radiol. 2004;33:196-201.

6. INfORM, International Network for Orofacial Pain and Related Disorders Methodology (Previously known as: International RDC TMD Consortium Network). Retrieved from https://ubwp.buffa lo.edu/rdc-tmdinternational/. Accessed January 20, 2020
7. Schiffman E, Ohrbach R, Truelove E, et al. Diagnostic Criteria for Temporomandibular Disorders (DC/TMD) for clinical and research applications: recommendations of the international RDC/TMD consortium network and orofacial pain special interest group. J Oral Facial Pain Headache. 2014;28:6-27.

8. Ahmad M, Hollender L, Anderson Q, et al. Research diagnostic criteria for temporomandibular disorders (RDC/TMD): development of image analysis criteria and examiner reliability for image analysis. Oral Surg Oral Med Oral Pathol Oral Radiol Endod. 2009;107:844-860.

9. Buchanan A, Thachil K, Haggard C, Kalathingal S. Predoctoral and postdoctoral education on cone-beam computed tomography. J Evid Based Dent Pract. 2017;17:310-316.

10. Lavanya R, Babu DB, Waghray S, Chaitanya NC, Mamatha B, Nithika $M$. A questionnaire cross-sectional study on application of $C B C T$ in dental postgraduate students. Pol J Radiol. 2016;81:181-189.

11. Francis B, Mauriello SM, Phillips C, Englebardt S, Grayden SK. Assessment of online continuing dental education in North Carolina. J Contin Educ Health Prof. 2000;20:76-84.

12. Clark GT. Education problems and Web-based teaching: how it impacts dental educators? J Am Coll Dent. 2001:68:25-34.

13. Mitov G, Dillschneider T, Abed MR, Hohenberg G, Pospiech P. Introducing and evaluating MorphoDent, a Web-based learning program in dental morphology. J Dent Educ. 2010;74:1133-1139.

14. Howerton WB Jr, Enrique PRT, Ludlow JB, Tyndall DA. Interactive computer-assisted instruction vs. lecture format in dental education. J Dent Hyg. 2004;78:10.

15. SEDENTEXCT Guidelines. Retrieved from http://www.sedentexct. eu/. Accessed January 202020

16. Hassan BA, Jacobs R, Scarfe WC, Al-Rawi WT. A web-based instruction module for interpretation of craniofacial cone beam CT anatomy. Dentomaxillofac Radiol. 2007;36:348-355.

17. Al-Rawi WT, Jacobs R, Hassan BA, Sanderink G, Scarfe WC. Evaluation of web-based instruction for anatomical interpretation in maxillofacial cone beam computed tomography. Dentomaxillofac Radiol. 2007;36:459-464.

18. Iskanderani D, Alstergren $P$, Hellén-Halme $K$. Assessment of a training programme on detection of temporomandibular joint osseous changes applying pre-defined 2D multiplane cone beam computed tomography reconstructions. J Oral Rehabil. 2018:45:282-288.

19. Altman DG. Practical statistics for medical research, 1st edn. London, UK: Chapman and Hall; 1991.

20. Hellen-Halme K, Hollender L, Janda M, Petersson A. Web-based calibration of observers using MRI of the temporomandibular joint. Dentomaxillofacial Radiol. 2012;41:656-661.

How to cite this article: Iskanderani D, Alstergren P, Ekberg EC, Shi X-Q, Hellén-Halme K. Web-based educational programme for temporomandibular joint assessment with cone-beam computed tomography. J Oral Rehabil. 2020;00:1-7. https://doi. org/10.1111/joor.13065 

III 



\title{
Dose distributions in adult and child head phantoms for panoramic and cone beam computed tomography imaging of the temporomandibular joint
}

\author{
Durer Iskanderani, BDS, MDSc, ${ }^{\mathrm{a}, \mathrm{b}}$ Mats Nilsson, PhD, ${ }^{\mathrm{a}, \mathrm{c}}$ Per Alstergren, DDS, PhD, ${ }^{\mathrm{d}}$ and \\ Kristina Hellén-Halme, DDS, $\mathrm{PhD}^{\mathrm{a}}$
}

\begin{abstract}
Objectives. The aim of this study was to map and compare the distributions of absorbed doses with Gafchromic film for panoramic radiography and cone beam computed tomography (CBCT) examinations of the temporomandibular joint (TMJ) by using adult and child phantoms.

Study Design. Gafchromic films were placed at 5 selected levels of anthropomorphic head phantoms of an adult and a child. Clinical protocols for panoramic and CBCT imaging of the TMJ were used for three 2-dimensional or 3-dimensional dental x-ray units. Mean absorbed doses in a set of radiosensitive tissues within the oral and maxillofacial regions were estimated.

Results. The absorbed doses varied considerably among and within radiosensitive tissues. The bone surface and the salivary glands received the highest absorbed doses compared with other tissues, in both panoramic and CBCT examinations of the TMJ. The radiation burden to the adult phantom was generally higher than that to the child phantom. Small right and left fields of view were associated with lower amounts of radiation, in contrast to a single larger field of view.

Conclusions. The absorbed dose within all radiosensitive tissues varied considerably in relation to examination type, $\mathrm{x}$-ray unit, clinical settings, and patient age. The mean doses were smaller when using 2 (bilateral) $4 \times 4 \mathrm{~cm}$ volumes than with use of one $14 \times 5 \mathrm{~cm}$ volume. (Oral Surg Oral Med Oral Pathol Oral Radiol 2020;130:200-208)
\end{abstract}

Radiography is a valuable and useful diagnostic tool that is widely used in dentistry. For verifying clinical findings and improving the quality of the diagnostic decision as well as the treatment plan, more and more radiographic examinations are being performed. As a result, total patient radiation dose has increased over time in combination with the presence of radiosensitive structures in the oral and maxillofacial region, raising concerns about the total delivered dose. This is potentially more risky for young patients whose developing tissues are more radiosensitive. ${ }^{1}$ Thus, radiographic examinations should hold radiation doses to a minimum level that still delivers the required diagnostic information, according to the ALADA (As Low As Diagnostically Acceptable) principle. ${ }^{2}$

In general dental practice, panoramic imaging often helps map the maxillofacial region and rule out odontogenic sources of disease or gross osseous alterations. ${ }^{3}$ However, the technique has poor reliability among observers and low sensitivity; that is, it underestimates the radiologic findings of disease. For this reason, it has

${ }^{\mathrm{a}}$ Department of Oral and Maxillofacial Radiology, Faculty of Odontology, Malmö University, Malmö, Sweden.

${ }^{\mathrm{b}}$ Department of Oral and Maxillofacial Radiology, Faculty of Dentistry, King Abdulaziz University, Jeddah, Saudi Arabia.

${ }^{\mathrm{c}}$ Department of Medical Radiation Physics, Skåne University Hospital, Malmö, Sweden.

${ }^{\mathrm{d} D e p a r t m e n t ~ o f ~ O r o f a c i a l ~ P a i n ~ a n d ~ J a w ~ F u n c t i o n, ~ F a c u l t y ~ o f ~ O d o n-~}$ tology, Malmö University, Malmö, Sweden.

Received for publication Jul 17, 2019; returned for revision Jan 4, 2020; accepted for publication Jan 11, 2020.

(C) 2020 The Author(s). Published by Elsevier Inc. This is an open access article under the CC BY-NC-ND license. (http:// creativecommons.org/licenses/by-nc-nd/4.0/)

2212-4403/\$-see front matter

https://doi.org/10.1016/j.0000.2020.01.003 limited use in investigating temporomandibular joint (TMJ)-related osseous changes ${ }^{4,5}$ and is not included in the diagnostic criteria for temporomandibular disorder (TMD). ${ }^{4}$ Three-dimensional TMJ imaging has been associated with better appreciation of TMJ anatomy and function. ${ }^{3}$ Thus, additional cone beam computed tomography (CBCT) imaging is recommended when detailed diagnostic information is required, further radiographic imaging is needed, or standard treatment has failed. Several previous studies have indicated the important contribution of $\mathrm{CBCT}$ imaging to TMJ diagnosis and treatment planning because of its high diagnostic accuracy and the relatively low radiation dose compared with other 3-dimensional (3-D) imaging techniques, such as multidetector computed tomography (CT) ${ }^{6-8}$ CBCT units, which offer varying spatial resolutions and radiation doses, provide clinicians with a variety of protocols and fields of view (FOVs) for imaging the TMJ.

The literature describes various dosimetric methods for evaluating radiation dose distributions. Gafchromic film dosimetry, in particular, is one of the more common methods for providing dose verification and for measuring dose maps with high spatial resolution, low

\section{Statement of Clinical Relevance}

Accurate measurement of the absorbed dose in the small radiation fields in dental radiology is challenging. The use of Gafchromic film has shown promising outcomes for mapping absorbed dose distributions, permitting dose comparisons between adults and children, and among different protocols. 
energy dependence, and adequate accuracy. ${ }^{9-11}$ Gafchromic film is a self-developing film and has a radiosensitive layer that contains a crystalline diacetylene monomer, which, when irradiated, polymerizes and darkens. ${ }^{12}$ The amount of darkening is related to the absorbed dose. ${ }^{12}$

Several studies have measured doses absorbed during radiographic TMJ examinations by using thermoluminescent dosimeters (TLDs). ${ }^{13-17}$ In organ dose measurements, TLDs are associated with limited spatial resolution; this is a disadvantage in situations with steep dose gradients. ${ }^{18}$ Gafchromic film has been suggested as a more feasible tool for radiation measurements, ${ }^{12,19,20}$ but only 1 report has described the use of Gafchromic film in TMJ dosimetry. ${ }^{20}$ The aim of this study was to map and compare the absorbed dose distributions with Gafchromic films in panoramic radiography and CBCT examinations of the TMJ by using adult and child phantoms.

\section{MATERIALS AND METHODS \\ Imaging units}

Absorbed dose distributions were measured by using 3 dental X-ray units from the same manufacturer (J. Morita Corp., Kyoto, Japan): Veraviewepocs 3-D F40, Veraview X800, and 3-D Accuitomo 170. Panoramic exposures were made with the Veraviewepocs 3-D F40 and Veraview X800 units using both the adult and child protocols with 220-degree rotation. CBCT volumes were centered on the TMJs bilaterally and made with the Veraviewepocs 3-D F40 and Veraview X800 units using both the adult and child protocols. The Veraviewepocs 3-D F40 was used at 180-degree (half) rotation (this unit is limited to 180-degree rotation), and exposures were made with the Veraview X800 unit at 360-degree (full) rotation and 180-degree (half) rotation with both units using a $4 \times 4 \mathrm{~cm}$ FOV. The 3-D Accuitomo 170 used adult and child protocols, each with full- and half-rotations, at $4 \times 4 \mathrm{~cm}$ and $14 \times 5 \mathrm{~cm}$ FOVs. All instrument settings were those commonly used in the clinic, according to the manufacturer's recommendation (Table I).

\section{Phantoms and Gafchromic films}

Two phantoms were used in dose distribution measurements: the head section of an adult anthropomorphic RANDO phantom, which was $175 \mathrm{~cm}$ in height and $73.5 \mathrm{~kg}$ in weight (The Phantom Laboratory, Salem, NY); and the head of a CIRS anthropomorphic phantom of an average 10-year-old child, $140 \mathrm{~cm}$ in height and $32 \mathrm{~kg}$ in weight (Computerized Imaging Reference Systems, Norfolk, VA). Both phantoms were cross-sectional in design, with 25-mm-thick sections, and consisted of a human skeleton (RANDO) or bone-equivalent material (CIRS) embedded in soft tissue-equivalent materials. For each adult phantom slice, the boundaries of the organs of interest were delineated by using an anatomy atlas. $^{21}$ At the time of this study, the literature had no cross-sectional anatomy atlas that could be consulted for a 10-year-old child, so an oral radiologist studied a number of anonymized CT examinations of 10-year-old children in the hospital image archive to estimate organ shape and location. These findings were correlated with the corresponding structures in the adult anatomy atlas. In general, the sites were similar, with minor differences among the slices.

Table I. Exposure parameters of the three dental x-ray units used for panoramic and CBCT examinations

\begin{tabular}{|c|c|c|c|c|c|}
\hline Protocol & Unit & $k V$ & $m A s$ & Rotation & $F O V$ \\
\hline 1. Panoramic adult & Veraviewepocs 3-D F40 & 70 & 74 & 220 & N/A \\
\hline 2. Panoramic child & Veraviewepocs 3-D F40 & 64 & 65 & 220 & N/A \\
\hline 3. Panoramic adult & Veraview X800 & 75 & 119 & 220 & N/A \\
\hline 4. Panoramic child & Veraview X800 & 75 & 104 & 220 & N/A \\
\hline 5. CBCT, TMJ B, adult & Veraviewepocs 3-D F40 & 90 & 47 & 180 & $4 \times 4$ \\
\hline 6. СBCT, TMJ B, child & Veraviewepocs 3-D F40 & 90 & 47 & 180 & $4 \times 4$ \\
\hline 7. CBCT, TMJ B, adult & Veraview X800 & 100 & 107 & 360 & $4 \times 4$ \\
\hline 8. CBCT, TMJ B, adult & Veraview X800 & 100 & 56 & 180 & $4 \times 4$ \\
\hline 9. CBCT, TMJ B, child & Veraview X800 & 100 & 90 & 360 & $4 \times 4$ \\
\hline 10. CBCT, TMJ B, child & Veraview X800 & 100 & 47 & 180 & $4 \times 4$ \\
\hline 11. CBCT, TMJ B, adult & 3-D Accuitomo 170 & 90 & 105 & 360 & $4 \times 4$ \\
\hline 12. CBCT, TMJ B, adult & 3-D Accuitomo 170 & 90 & 54 & 180 & $4 \times 4$ \\
\hline 13. CBCT, TMJ B, child & 3-D Accuitomo 170 & 90 & 105 & 360 & $4 \times 4$ \\
\hline 14. CBCT, TMJ B, child & 3-D Accuitomo 170 & 90 & 54 & 180 & $4 \times 4$ \\
\hline 15. CBCT, TMJ B, adult & 3-D Accuitomo 170 & 90 & 105 & 360 & $14 \times 5$ \\
\hline 16. CBCT, TMJ B, adult & 3-D Accuitomo 170 & 90 & 54 & 180 & $14 \times 5$ \\
\hline 17. CBCT, TMJ B, child & 3-D Accuitomo 170 & 90 & 105 & 360 & $14 \times 5$ \\
\hline 18. CBCT, TMJ B, child & 3-D Accuitomo 170 & 90 & 54 & 180 & $14 \times 5$ \\
\hline
\end{tabular}

$B$, bilateral; $C B C T$, cone beam computed tomography; $F O V$, field of view; $k V$, kilovolt; mAs, milliampere-seconds; $N / A$, not applicable; $T M J$, temporomandibular joint. 
Gafchromic XR-QA2 film (ISP Corp., Wayne, NJ) was used as the dose measurement tool. Because the Gafchromic film response to absorbed dose is nonlinear, it was calibrated against a Radcal $10 \times 6-6$ ionization chamber (Radcal Corporation, Monrovia, CA). ${ }^{12}$ The film was exposed to 8 doses of radiation between 2000 and 110,000 $\mu \mathrm{Gy} ; 1$ nonirradiated film was used for calculating the background value. In a previous study, ${ }^{11}$ the film was calibrated for kilovolt $(\mathrm{kV})$ settings from 60 to $120 \mathrm{kV}$ by using the same ionization chamber (which has a flat energy response in this interval). It was found that the calibration curves coincided. Therefore, the same calibration curve could be used for all exposure settings. All films were scanned into 24-bit RGB images (8-bit per channel) on an Epson Perfection 4990 Photo flatbed scanner (Seiko Epson Corp., Nagano, Japan) with a consistent orientation by using a template for the shape of each phantom level.

The images were then converted into 8-bit gray scale and analyzed with ImageJ software (National Institutes of Health, Bethesda, MD) to calculate net pixel values (pixel value in the exposed image minus the mean pixel value in the background film) to plot a dose-response calibration curve (Figure 1). Before applying the dose calibration equation to the net pixel value data, these were converted to 32-bit floating point data to avoid truncation errors.

\section{Radiation dose measurements}

Scout images of the phantoms were made to ensure correct and reproducible positioning within the selected FOV, with the sectional planes parallel to the floor. Gafchromic films were loaded into 5 levels of the phantoms. For panoramic examination, 1 film was centered at the occlusal level, and 2 films were positioned superiorly and 2 inferiorly. For the TMJ examination, 1 film was centered on the TMJ region, and 2 films were positioned superiorly and 2 inferiorly. For each examination protocol, the central beam of all radiation fields was deliberately placed

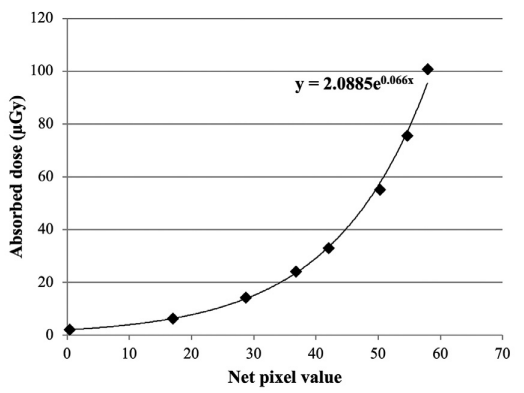

Fig. 1. Dose response calibration curve for Gafchromic XRQA2 film, Lot \# 07271701 (ISP Corp., Wayne, NJ).
$1 \mathrm{~cm}$ away from the central level where a film was placed, thus avoiding irradiating the film with a parallel beam (Figure 2). Multiple exposures were made to obtain a dose that was within the dynamic range of the Gafchromic films (see Table I). After reading the image into the ImageJ program and subtracting the background value, we converted the net pixel value matrix to absorbed dose by using the dose-response curve. The absorbed dose matrix was then divided by the number of exposures, which provided an absorbed dose matrix for 1 exposure, that is, the clinical situation. Figure 3 illustrates the steep dose gradients in 1 panoramic and $2 \mathrm{CBCT}$ examinations.

The mean doses absorbed by the radiosensitive structures within the oral and maxillofacial region (brain, eyes, salivary glands, oral mucosa, and bone surface) were estimated by overlapping the corresponding regions of interest on the dose distribution matrices and calculating the mean absorbed dose inside each region of interest. This was repeated for all film sheets from both phantoms. For large structures lying in multiple phantom levels, the mean values were calculated by the mean of the mean values in which the structure was present.
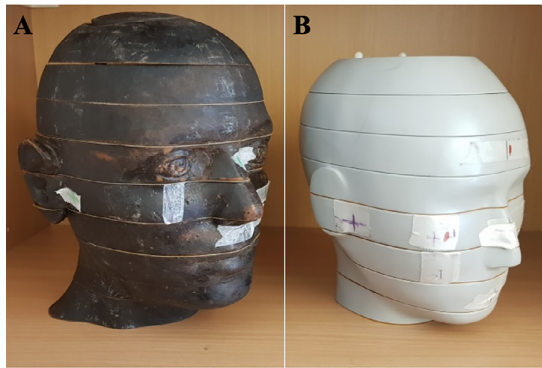

C

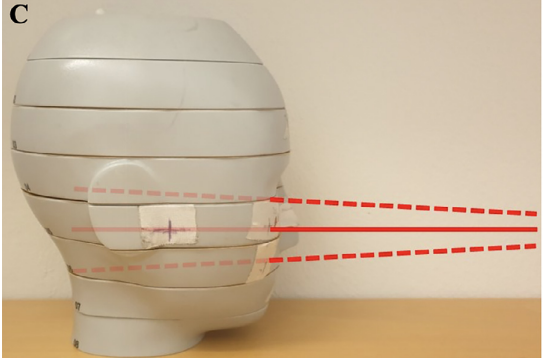

Fig. 2. Phantoms loaded with Gafchromic films on 5 levels. A, Adult phantom, for temporomandibular joint examination. B, Child phantom, for panoramic examination, as indicated by the numbers. $\mathbf{C}$, Child phantom with the central x-ray beam placed $1 \mathrm{~cm}$ away from the central level where a film was placed, thus avoiding irradiating the film with a parallel beam. 


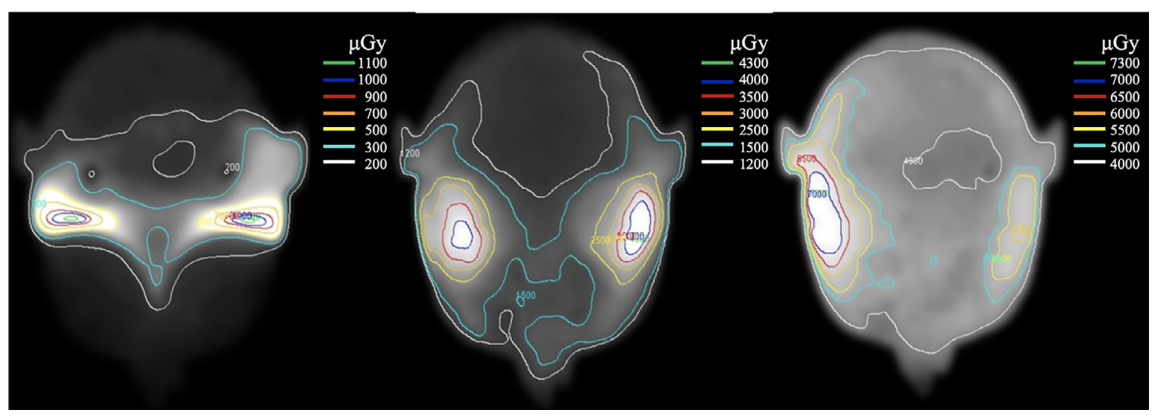

Fig. 3. Absorbed dose distributions are given as isodose lines in microgray for an adult phantom (left to right): A panoramic examination and temporomandibular joint (TMJ) examinations, using two $4 \times 4 \mathrm{~cm}$ and one $14 \times 5 \mathrm{~cm}$ cone beam computed tomography (CBCT) volumes.

Deriving the absorbed dose to bone surface needs special attention. The higher atomic number for bone will result in a higher absorbed dose value at a point than it would have been for soft tissue. This can be accounted for by multiplying the measured dose value with the ratio of the mass energy absorption coefficients, $\mu_{\mathrm{en}} / \rho$, for bone and soft tissue, respectively. ${ }^{22}$ As this ratio is strongly dependent on photon energy, it has to be evaluated for the different situations at hand. The mean photon energies for the $\mathrm{x}$-ray spectra using $\mathrm{kV}$ (see Table I) and total filtration (Table II) were calculated, ${ }^{23}$ representing the $\mathrm{x}$-ray spectrum impinging on the phantom. In addition, the mean photon energies after penetration of $100 \mathrm{~mm}$ of soft tissue were calculated. The mean of these 2 values was used for estimating the ratio of the mass energy absorption coefficients, which were retrieved from Evans. ${ }^{24}$ For the $\mathrm{x}$-ray spectra in question, the ratio values varied from 3.0 to 4.1.

The values obtained were comparable for the different x-ray units, phantoms, and protocols. For convenience, the values in Tables III through VI were normalized to one of the protocols, and this allowed for calculation of relative radiation burden percentages.

Table II. Parameters affecting the depth dose characteristics for the 3 CBCT units used in this study

\begin{tabular}{llll}
\hline Unit & $\begin{array}{l}\text { Isocenter distance } \\
(\mathrm{mm})\end{array}$ & $\begin{array}{l}\text { Total filtration } \\
(\mathrm{mm} \mathrm{Al})\end{array}$ & $\mathrm{kV}$ \\
\hline $\begin{array}{l}\text { Veraviewepocs } \\
\text { 3-D F40 }\end{array}$ & 340 & 9.5 & 90 \\
Veraview X800 & 400 & 11 & 100 \\
$\begin{array}{l}\text { 3-D Accuitomo } \\
\text { 170 }\end{array}$ & 540 & 4.7 & 90 \\
\hline
\end{tabular}

$C B C T$, cone beam computed tomography; $k V$, kilovolt.

\section{Measurement uncertainty}

The overall error of the absorbed dose estimation in 1 single pixel, $\varepsilon$, is caused by:

1. Uncertainty of the calibration of the ionization chamber. For an instrument of this type it is typically in the order of $\pm 10 \%$.

2. Uncertainty of determining the net pixel value in the 8-bit images for the dose calibration curve. As the pixel values in a uniformly irradiated film follow a normal distribution, this error (standard error of measurement) is around $\pm 1.5 \%$.

3. Uncertainty of the curve fitting to the dose points (see Figure 1). This is around $\pm 8 \%$.

4. Uncertainty of determining the pixel value as a result of truncation errors in the point where the dose is calculated. This error is around $\pm 2 \%$.

Table III. Mean absorbed doses for the organs of interest in the panoramic examinations of an adult and a child phantom with two 2-D/3-D combined dental imaging units (J. Morita Corp., Kyoto, Japan)

\begin{tabular}{llllll}
\hline \multirow{2}{*}{ Organ } & \multicolumn{3}{c}{ Mean absorbed organ doses $(\mu G y)$} \\
\cline { 2 - 3 } \cline { 5 - 6 } & Veraviewepocs 3-D F40 & & Veraview X800 \\
\cline { 2 - 3 } \cline { 5 - 6 } & Adult & Child & & Adult & Child \\
\hline Brain & 150 & 80 & & 210 & 150 \\
Eyes & 100 & 70 & & 140 & 110 \\
Salivary glands & 700 & 660 & & 850 & 940 \\
Oral mucosa & 250 & 190 & & 260 & 270 \\
Bone surface & 1440 & 980 & & 1670 & 1480 \\
Relative radiation & $100^{*}$ & 67 & 114 & 104 \\
burden \% & & & & \\
\hline
\end{tabular}

*Normalization value for panoramic examinations. $\mu G y$, microgray. 
Accordingly, the overall uncertainty of the absorbed dose estimation in 1 single point, $\varepsilon$, can be estimated to $\pm 13 \%$. However, all results presented in this paper are mean doses to different organs and tissues. The mean doses are calculated from thousands of measurement points. Therefore, the overall error of the mean values given in Tables III through VI is $\frac{\varepsilon}{\sqrt{n}}$, where $n$ is the total number of pixels used to calculate the mean absorbed dose. The uncertainties of the mean values are, therefore, very small. This applies even more to the integration of the radiation burden, which is carried out over millions of measurement points.

\section{RESULTS}

Table III shows the mean absorbed dose for each organ of interest in panoramic examinations of the adult and child phantoms with the 2 dental $x$-ray units that could produce panoramic images. Tables IV and V present mean absorbed doses in CBCT examinations of the same organs of interest in the adult and child phantoms using full (see Table IV) and half (see Table V) rotations with 2 and 3 dental x-ray units, respectively. In all examinations, the highest absorbed doses occurred in the bone surface and the salivary glands for both the panoramic and the CBCT examinations. The other organs and tissues received $270 \mu \mathrm{Gy}$ or less during the panoramic examination and $1530 \mu \mathrm{Gy}$ or less in a small FOV of the full- and half-rotation CBCT scans. The radiation burden to the adult phantom was generally higher than that for the child phantom.

For both panoramic and full-rotation CBCT examinations, Veraview X800 delivered the highest radiation burden compared with the other units (see Tables III and IV). In contrast, 3-D Accuitomo 170 delivered the lowest radiation burden during the CBCT examinations, at both fulland half-rotation angles (see Tables IV and V). The

Table IV. Mean absorbed doses for the organs of interest in full-rotation CBCT examinations of an adult phantom and a child phantom with 2 dental imaging units (J. Morita Corp., Kyoto, Japan)

\begin{tabular}{|c|c|c|c|c|}
\hline \multirow[t]{3}{*}{ Organ } & \multicolumn{4}{|c|}{ Mean absorbed organ doses $(\mu G y)$} \\
\hline & \multicolumn{2}{|c|}{ Veraview $X 800$} & \multicolumn{2}{|c|}{ 3-D Accuitomo 170} \\
\hline & Adult & Child & Adult & Child \\
\hline Brain & 950 & 680 & 680 & 570 \\
\hline Eyes & 570 & 1530 & 870 & 1210 \\
\hline Salivary glands & 3510 & 3070 & 1960 & 2210 \\
\hline Oral mucosa & 610 & 480 & 430 & 380 \\
\hline Bone surface & 5880 & 6030 & 4390 & 5150 \\
\hline Relative radiation burden $\%$ & $100^{*}$ & 94 & 78 & 73 \\
\hline
\end{tabular}

*Normalization value for $\mathrm{CBCT}$ examinations: full-rotation. $C B C T$, cone beam computed tomography; $\mu G y$, microgray.
Veraviewepocs 3-D F40 unit showed the highest radiation burden of the 3 units for half-rotation scans (see Table V).

Table VI presents the mean organ dose for the adult and child phantoms at different FOVs for CBCT imaging with the Accuitomo 170 unit. The mean organ dose was lower in 2 small FOVs than in 1 large FOV with only a few exceptions. When comparing these 2 situations, we also found a $27 \%$ lower relative radiation burden in the bilateral $4 \times 4$ FOVs compared with one $14 \times 5$ FOV in a 360-degree rotation for both phantoms, whereas $16 \%$ and $22 \%$ reductions were found with a 180-degree rotation for the adult and child phantoms, respectively (Figure 4).

\section{DISCUSSION}

We chose not to report our results as effective dose. The reason for this is that effective dose is often-and increasingly - not used for its actual purpose. ${ }^{25-27}$ The medical community must use the effective dose concept wisely, realizing that an effective dose estimate represents a generic estimate of risk from a given procedure to a generic model of the human body. In no way does it represent risk to any one individual. Effective dose should not be used for epidemiologic studies or the estimation of population risks because of the inherent uncertainty and oversimplification involved. ${ }^{27}$ This is especially true for children, in our case the phantom for a 10-year-old child. In such a situation, effective dose is not even defined because of lack of data for estimating weighting factors.

The International Commission on Radiologic Protection is aware of and concerned about the widespread misuse of the concept of effective dose and is presently investigating if other quantities can be used or developed as a replacement. Ongoing research aims at introducing alternative ways of estimating radiation risk; for instance, from the use of dose area product measurements together with conversion factors that depend on which body part is irradiated. Dose area product measurements give readings that are proportional to the energy imparted to a certain region of the body. This is why we used the term "radiation burden" in the paper because it also is proportional to the energy imparted.

One attractive method for comparing the radiation burden to the patient in relation to different $\mathrm{x}$-ray units, protocols, and patient ages would have been to use the concept of energy imparted. The definition of energy imparted $(\varepsilon)$ to matter in a given volume is the sum of all energy deposits in the volume; thus, $\epsilon=\sum_{\mathrm{i}} \epsilon_{\mathrm{i}}$, where the summation is performed over all energy deposits, $\varepsilon_{\mathrm{i}}$, in that volume. However, we did not have absorbed dose values over the whole volume, only for 5 slices in that volume. By integrating the absorbed dose values in the 5 slices, we would get a useful quantity that represents the "radiation burden" to the patient and which also would be roughly proportional to the energy imparted. 
Table V. Mean absorbed doses for the organs of interest in half-rotation CBCT examinations of an adult phantom and a child phantom with 3 dental imaging units (J. Morita Corp., Kyoto, Japan)

\begin{tabular}{|c|c|c|c|c|c|c|}
\hline \multirow[t]{3}{*}{ Organ } & \multicolumn{6}{|c|}{ Mean absorbed organ doses $(\mu G y)$} \\
\hline & \multicolumn{2}{|c|}{ Veraviewepocs 3-D F40 } & \multicolumn{2}{|c|}{ Veraview $X 800$} & \multicolumn{2}{|c|}{ 3-D Accuitomo 170} \\
\hline & Adult & Child & Adult & Child & Adult & Child \\
\hline Brain & 760 & 560 & 680 & 520 & 480 & 410 \\
\hline Eyes & 350 & 340 & 250 & 370 & 240 & 420 \\
\hline Salivary glands & 2270 & 1900 & 1740 & 1810 & 1180 & 1300 \\
\hline Oral mucosa & 320 & 240 & 340 & 250 & 200 & 190 \\
\hline Bone surface & 3960 & 3330 & 3390 & 3090 & 2560 & 2660 \\
\hline Relative radiation burden $\%$ & $100^{*}$ & 81 & 82 & 78 & 61 & 60 \\
\hline
\end{tabular}

*Normalization values for CBCT examinations: half-rotation.

$C B C T$, cone beam computed tomography; $\mu G y$, microgray.

Table VI. Mean absorbed doses for the organs of interest for adult phantoms and child phantoms when comparing different fields of view for cone beam computed tomography imaging with the 3-D Accuitomo 170 unit (J. Morita Corp., Kyoto, Japan)

\begin{tabular}{|c|c|c|c|c|c|c|c|c|}
\hline \multirow{4}{*}{$\begin{array}{l}\text { Organ } \\
\text { FOV }\end{array}$} & \multicolumn{8}{|c|}{ Mean organ doses $(\mu G y)$} \\
\hline & \multicolumn{4}{|c|}{ Adult phantom } & \multicolumn{4}{|c|}{ Child phantom } \\
\hline & \multicolumn{2}{|c|}{360 -degree rotation } & \multicolumn{2}{|c|}{ 180-degree rotation } & \multicolumn{2}{|c|}{360 -degree rotation } & \multicolumn{2}{|c|}{ 180-degree rotation } \\
\hline & $2(4 \times 4)$ & $14 \times 5$ & $2(4 \times 4)$ & $14 \times 5$ & $2(4 \times 4)$ & $14 \times 5$ & $2(4 \times 4)$ & $14 \times 5$ \\
\hline Brain & 1360 & 2220 & 950 & 1460 & 1150 & 1590 & 810 & 1100 \\
\hline Eyes & 1740 & 2290 & 480 & 340 & 2430 & 4650 & 850 & 870 \\
\hline Salivary glands & 3930 & 4400 & 2370 & 1810 & 4420 & 4630 & 2610 & 2440 \\
\hline Oral mucosa & 870 & 1530 & 400 & 580 & 760 & 1390 & 390 & 530 \\
\hline Bone surface & 8820 & 11160 & 5110 & 4930 & 10290 & 12490 & 5290 & 6080 \\
\hline Relative radiation burden $\%$ & $100^{*}$ & 138 & 54 & 65 & 94 & 128 & 53 & 68 \\
\hline
\end{tabular}

*Normalization value for comparison of $2(4 \times 4)$ with $14 \times 5$ FOVs. $F O V$, field of view.

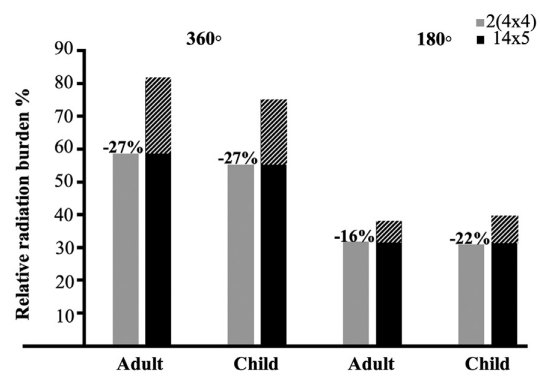

Fig. 4. The percentage of relative radiation burden for adult and child phantoms when comparing different fields of view (FOVs) for cone beam computed tomography (CBCT) imaging (Accuitomo 170, J. Morita Corp., Kyoto, Japan).

In this investigation, we measured radiation burden associated with various examinations by using a variety of imaging techniques, x-ray units, examination settings, FOVs, and phantoms. Because the main purpose of panoramic radiography during TMJ assessment is to rule out odontogenic causes, the standard panoramic radiography mode was included. In panoramic imaging, the Veraviewepocs 3-D F40 unit produced a lower radiation burden compared with the Veraview X800 unit. The most likely reason was that the kilovoltage and milliamperage values were significantly higher for Veraview X800. The higher filtration of Veraview X800 obviously did not fully compensate for this.

Generally, all studied tissues were exposed to considerably more radiation in CBCT examinations compared with panoramic examinations, and this finding is consistent with the literature. ${ }^{20,28,29}$ In the various protocols used in this study, the bone surface and salivary glands received the highest absorbed doses, and this finding also is in agreement with reports from previous studies. ${ }^{13,14,20,30}$ The bone surface shows high absorbed dose values because of the great attenuation of $\mathrm{x}$-rays by bone. The location of the salivary gland tissues - in the center of rotation during x-ray beam and detector movement for both panoramic and CBCT examinations-explains the high absorbed dose of this organ. Even if most of the scanned 
anatomy is only momentarily irradiated, structures at the rotational centers are exposed longer or continuously.

An interesting finding in this study was that between the adult and child phantoms, the radiation burden was significantly larger with the Veraviewepocs 3-D F40 unit than with the Veraview X800 unit when used with the manufacturer's default settings for the supposed patient ages of the phantoms. We believe that the more compact design of the Veraviewepocs 3-D F40 unit with an X-ray focal spot-center of rotation distance of $340 \mathrm{~mm}$, versus $400 \mathrm{~mm}$ for the Veraview X800 unit, was the reason for the larger difference in radiation burden. For the same dose at the surface of the detector, the effect of the inverse square law will be more pronounced when the distance to the x-ray focal spot is $14 \%$ to $36 \%$ less, resulting in higher doses and greater radiation burden.

Differences in the locations and sizes of the organs could also contribute to differences between the phantoms. Wahid et al. ${ }^{31}$ and $\mathrm{Al}$ Najjar et al. ${ }^{32}$ reported ambiguous results when comparing absorbed doses to organs and tissues in adult and child phantoms; that is, they found both higher and lower doses for adults compared with children. Moreover, Choi and Ford ${ }^{33}$ reported higher absorbed doses for a child phantom compared with the adult with use of an ionization chamber.

The depth dose characteristics for the TMJ protocols depend on (1) the attenuation of the primary beam (in its dependence on kilovoltage and filtration); (2) the focal spot-isocenter distance (inverse square law); and (3) the buildup of scatter radiation. The 3 units studied were indeed very different with regard to these parameters, as summarized in Table II.

The greater decrease in depth dose as a result of the shorter isocenter distance for the Veraviewepocs 3-D F40 and Veraview X800 units than for the 3-D Accuitomo 170 unit is compensated for by the much higher filtration, making the primary attenuation lower; the extent of this effect could be studied by using measurements in a homogeneous water phantom.

Because of these construction differences, it is not evident whether the doses to the child phantom, in general, should be larger than those to the adult phantom. For instance, as can be seen in Table VI, the mean dose to the brain is higher for the adult, probably because the adult phantom is larger and the distance from the $\mathrm{x}$-ray source to the entrance point of the phantom, especially at the back of the head, is approximately $20 \mathrm{~mm}$ shorter than for the child phantom, resulting in a higher entrance dose.

The smaller dimensions of the child phantom can affect the dosage compared with those of the adult phantom for other reasons. For instance, the eye dose for a full rotation was significantly higher for the child phantom (see Table IV); this is because the eye of the child is closer to the irradiated $4 \times 4 \mathrm{~cm}$ volume.
The 3-D Accuitomo 170 unit has an even longer distance $(540 \mathrm{~mm})$ between the $\mathrm{x}$-ray source and the center of rotation. Accordingly, the radiation burden was highest for the Veraviewepocs 3-D F40 unit, followed by the Veraview X800 unit; the 3-D Accuitomo 170 unit had the lowest radiation burden (see Table V). A notable finding was that organ doses generally were not halved when using the half-rotation protocol instead of the fullrotation protocol. The reason is the position of the organs, for example, the eyes, in relation to the start and stop angles of a half rotation.

As an independent method for comparison, we used the PCXMC Monte Carlo program ${ }^{34}$ to verify some of our measurements. To calculate the doses for the rotational geometry of the CBCT units, we used the PCXMC20 Rotation module supplied with the program. Focal spot-isocenter distance, field size, kilovoltage, and measured filtration values were entered for the dose calculations. The measured kilovoltage, filtration, and output of the $\mathrm{x}$-rays impinging on the phantom were almost unaffected by position over the $4 \times 4 \mathrm{~cm}$ radiation field. We used the mean dose to the salivary glands for the comparison (see Table IV). For the 3-D Accuitomo 170 unit, the measured mean dose for the child was $12 \%$ higher than that for the adult. The Monte Carlo calculations resulted in a $9 \%$ higher dose for the child. For the Veraview X800 unit, the corresponding numbers were $13 \%$ lower (measured) and $10 \%$ lower (calculated) for the child compared with the adult.

Our results clearly indicate that with the same exposure parameters, the use of 2 separate small FOVs (1 for each TMJ) generally results in lower doses and radiation burden compared with 1 large FOV that captures both TMJs simultaneously. Furthermore, a smaller voxel size associated with the smaller FOV could lead to better image quality. Nascimento et al. ${ }^{13}$ reported a similar reduction in radiation burden. Lukat et al. ${ }^{16}$ found similar results, although they compared different units.

In this study, we used 2 different phantoms that were available in our institution. Dosimetric methods that can be used in the present situation are those based on TLDs, optically stimulated luminescence dosimeters, or self-developing films, such as the Gafchromic film. However, use of LiF or aluminum oxide dosimeters in a soft tissue environment will create a problem because these dosimeters have a pronounced energy dependence in a 30-150 kiloelectron-volt interval because of their higher atomic number. ${ }^{35,36}$ Because the $\mathrm{x}$-ray spectrum varies with the position in the phantom, the actual absorbed doses in the phantom where such dosimeters were placed would be very difficult to estimate. Hourdakis and Nowotny ${ }^{37}$ expressed their concerns about the use of LiF and aluminum oxide dosimeters in radiation fields with spatially varying $\mathrm{X}$-ray spectra. 
The radiosensitive material in the Gafchromic film is diacetylene. The mass-energy absorption coefficient, $\mu_{\mathrm{en}} / \rho$, calculated by using National Institute of Standards and Technology data ${ }^{38}$ for this material, has an energy dependence perfectly parallel to that of water (soft tissue), making it suitable as a dosimeter because it has the same energy absorption characteristics as its surrounding material. Compared with air, the $\mu_{\mathrm{en}} / \rho$ characteristics coincide, which explains why the calibration curves for different kilovolt values are the same. This notion was supported by Hourdakis and Nowotny. ${ }^{37}$ The high spatial resolution of the film is another desirable property for mapping dose distributions in radiation fields with steep dose gradients, as is the case in dental radiology. Placing a TLD $\pm 3 \mathrm{~mm}$ from a point in the gradient can result in up to $85 \%$ variation in the measured signal from the dosimeter. Indeed, most of the radiation dosimeters are limited in that the organ dose depends on loading the dosimeters in manufacturer-determined locations in the phantom as a representative of the whole organ volume.

For the Gafchromic film, using the red channel of the RGB 24-bit images will give a more sensitive system (a steeper calibration curve). This also means that a small variation in pixel values in the high end of the usable interval will give a large increase in absorbed dose. We preferred to extend the dose measurement range instead by converting the RGB image to an 8-bit gray scale. Moreover, use of multiple color channels to convert the scanned images on the Gafchromic film into dose maps compensates for many of the common disturbances that are usually associated with this dosimetric method, such as the nonuniform thickness of the film's active layer, thus adding to data integrity and dosimetric accuracy. ${ }^{39}$

The currently available $\mathrm{x}$-ray units with their different settings are usually associated with varying amounts of radiation. Because of the differences in their construction, it is important to make the dose measurements specific to each unit to estimate patient radiation doses. Therefore, interpretation and comparison of dosimetric studies must be made with caution.

Weighing the risks and benefits of exposure to ionizing radiation is a vital matter to be considered carefully, particularly for children, as their developing tissues are more radiosensitive than adult tissues and their longer life expectancy increases the lifetime risk for developing radiation-induced cancers. Furthermore, selection of the proper radiographic examinations with the most appropriate clinical setting, as well as use of low dose protocols, is important to ensure diagnostic accuracy while adhering to the $\mathrm{ALADA}^{2}$ principle.

\section{CONCLUSIONS}

The absorbed doses within and between radiosensitive organs and tissues varied widely. Mean absorbed dose to the brain, eyes, salivary glands, oral mucosa, and bone surface varied considerably between adult and child phantoms. The bone surface and the salivary glands received the highest absorbed doses compared with other tissues, both in panoramic radiography and CBCT of the TMJ. The radiation burden to the adult phantom was generally higher than for the child phantom. The mean doses measured were smaller when using 2 (bilateral) $4 \times 4 \mathrm{~cm}$ volumes than for one $14 \times 5 \mathrm{~cm}$ volume.

\section{DISCLOSURE}

The authors declare no conflicts of interest.

\section{FUNDING}

This study was funded by King Abdulaziz University.

\section{ACKNOWLEDGMENT}

We are grateful to radiographer Curt Johansson for his help during the practical part of this study.

\section{REFERENCES}

1. Demeter S, Applegate KE, Perez M. Internet-based ICRP resource for healthcare providers on the risks and benefits of medical imaging that uses ionising radiation. Ann ICRP. 2016; $45: 148-155$.

2. Bushberg JT. Science, radiation protection, and the NCRP: building on the past, looking to the future. In: Proceedings of the fiftieth annual meeting program of NCRP: achievements of the past 50 years and addressing the needs of the future, March 10-11; 2014. Bethesda, MD. pp. 5-7.

3. Lewis EL, Dolwick MF, Abramowicz S, Reeder SL. Contemporary imaging of the temporomandibular joint. Dent Clin North Am. 2008;52:875-890

4. Ahmad M, Hollender L, Anderson Q, et al. Research diagnostic criteria for temporomandibular disorders (RDC/TMD): development of image analysis criteria and examiner reliability for image analysis. Oral Surg Oral Med Oral Pathol Oral Radiol Endod. 2009;107:844-860

5. Schmitter M, Gabbert O, Ohlmann B, et al. Assessment of the reliability and validity of panoramic imaging for assessment of mandibular condyle morphology using both MRI and clinical examination as the gold standard. Oral Surg Oral Med Oral Pathol Oral Radiol Endod. 2006;102:220-224.

6. Zain-Alabdeen EH, Alsadhan RI. A comparative study of accuracy of detection of surface osseous changes in the temporomandibular joint using multidetector CT and cone beam CT. Dentomaxillofac Radiol. 2012;41:185-191.

7. Honda K, Larheim TA, Maruhashi K, Matsumoto K, Iwai K. Osseous abnormalities of the mandibular condyle: diagnostic reliability of cone beam computed tomography compared with helical computed tomography based on an autopsy material. Dentomaxillofac Radiol. 2006;35:152-157

8. Tsiklakis K, Syriopoulos K, Stamatakis HC. Radiographic examination of the temporomandibular joint using cone beam computed tomography. Dentomaxillofac Radiol. 2004;33:196-201.

9. Devic S. Radiochromic film dosimetry: past, present, and future. Phys Med. 2011:27:122-134.

10. Butson MJ, Yu PKN, Cheung T, Alnawaf H. Energy response of the new EBT2 radiochromic film to $\mathrm{x}$-ray radiation. Radiat Meas. 2010;45:836-839. 
11. Hellen-Halme K, Nilsson M. The effects on absorbed dose distribution in intraoral $\mathrm{x}$-ray imaging when using tube voltages of 60 and $70 \mathrm{kV}$ for bitewing imaging. J Oral Maxillofac Res. 2013;4:e2.

12. Watanabe Y, Patel G, Patel P. Evaluation of a new self-developing instant film for imaging and dosimetry. Radiat Prot Dosimetry. 2006;120:121-124.

13. Nascimento HAR, Andrade MEA, Frazão MAG, Nascimento EHL, Ramos-Perez FMM, Freitas DQ. Dosimetry in CBCT with different protocols: emphasis on small FOVs including exams for TMJ. Braz Dent J. 2017;28:511-516.

14. Kadesjö N, Benchimol D, Falahat B, Näsström K, Shi X. Evaluation of the effective dose of cone beam CT and multislice CT for temporomandibular joint examinations at optimized exposure levels. Dentomaxillofac Radiol. 2015;44:20150041.

15. Oliveira MVL, Andrade MEA, Batista WO, Campos PSF. Skin doses on the lens for temporomandibular joint exam in cone beam computed tomography. Braz Arch Biol Technol. 2015;58:886-890.

6. Lukat T, Wong J, Lam E. Small field of view cone beam CT temporomandibular joint imaging dosimetry. Dentomaxillofac Radiol. 2013;42:20130082.

17. Librizzi ZT, Tadinada AS, Valiyaparambil JV, Lurie AG, Mallya SM. Cone-beam computed tomography to detect erosions of the temporomandibular joint: effect of field of view and voxel size on diagnostic efficacy and effective dose. Am J Orthod Dentofacial Orthop. 2011;140:e25-e30.

18. Ptaszkiewicz M, Braurer-Kirsch E, Klosowski M, Czopyk L, Olko P. TLD dosimetry for microbeam radiation therapy at the European Synchrotron Radiation Facility. Radiat Meas. 2008;43:990-993

19. Rampado O, Bianchi SD, Cornetto AP, Rossetti V, Ropolo R Radiochromic films for dental CT dosimetry: a feasibility study. Phys Med. 2014;30:18-24.

20. Al-Okshi A, Nilsson M, Petersson A, Wiese M, Lindh C. Using GafChromic film to estimate the effective dose from dental cone beam CT and panoramic radiography. Dentomaxillofac Radiol. 2013:42:20120343.

21. Möller T, Reif E. Cross-Sectional Anatomy. Pocket Atlas. CT and MRI. Volume1: Head, Neck, Spine, and Joints. New York: Thieme Medical Publishers; 1994.

22. Burlin TE. Cavity chamber theory. In: Attix FH, Roesch WC, eds. Radiation Dosimetry. Volume I: Fundamentals, Cambridge, MA: Academic Press; 1968. p. 362.

23. Poludniowski G, Landry G, DeBlois F, Evans P, Verhaegen F. SpekCalc: a program to calculate photon spectra from tungsten anode x-ray tubes. Phys Med Biol. 2009;54:N433-N438.

24. Evans RD. X-ray and $\gamma$-ray interactions. In: Attix FH, Roesch WC, eds. Radiation Dosimetry. Volume I: Fundamentals, Cambridge, MA: Academic Press; 1968. p. 135.

25. Brenner DJ. Effective dose: a flawed concept that could and should be replaced. Br J Radiol 2008:81:521-523.

26. Brenner DJ. We can do better than effective dose for estimating or comparing low-dose radiation risks. Ann ICRP. 2012;41:124-128.

27. McCollough CH, Christner JA, Kofler JM. How effective is effective dose as a predictor of radiation risk? AJR Am J Roentgenol. 2010;194:890-896.
28. Grünheid T, Schieck JRK, Pliska BT, Ahmad M, Larson BE. Dosimetry of a cone-beam computed tomography machine compared with a digital $\mathrm{x}$-ray machine in orthodontic imaging. Am J Orthod Dentofacial Orthop. 2012;141:436-443.

29. Ludlow JB, Davies-Ludlow L, Brooks S, Howerton W. Dosimetry of $3 \mathrm{CBCT}$ devices for oral and maxillofacial radiology: $\mathrm{CB}$ Mercuray, NewTom $3 \mathrm{G}$ and i-CAT. Dentomaxillofac Radiol. 2006:35:219-226

30. Ludlow JB, Davies-Ludlow LE, White SC. Patient risk related to common dental radiographic examinations: the impact of 2007 International Commission on Radiological Protection recommendations regarding dose calculation. I Am Dent Assoc. 2008; $139: 1237-1243$

31. Wahid MA, Choi E, MacDonald DS, Ford NL. Dosimetry analysis of panoramic-imaging devices in different-sized phantoms. J Appl Clin Med Phys. 2017;18:197-205.

32. Al Najjar A, Colosi D, Dauer LT, et al. Comparison of adult and child radiation equivalent doses from 2 dental cone-beam computed tomography units. Am J Orthod Dentofacial Orthop. 2013:143:784-792

33. Choi E, Ford NL. Measuring absorbed dose for i-CAT CBCT examinations in child, adolescent and adult phantoms. Dentomaxillofac Radiol. 2015;44:20150018.

34. Tapiovaara M, Siiskonen T. PCXMC: A Monte Carlo Program for Calculating Patient Doses in Medical X-ray Examinations. 2nd ed. Helsinki, Finland: Radiation and Nuclear Safety Authority; 2008 Report STUK-A231.

35. Mobit P, Agyingi E, Sandison G. Comparison of the energyresponse factor of $\mathrm{LiF}$ and $\mathrm{Al}_{2} \mathrm{O}_{3}$ in radiotherapy beams. Radiat Prot Dosimetry. 2006;119:497-499.

36. Agyingi EO, Mobit PN, Sandison GA. Energy response of an aluminium oxide detector in kilovoltage and megavoltage photon beams: an EGSnrc Monte Carlo simulation study. Radiat Prot Dosimetry. 2006;118:28-31.

37. Hourdakis J, Nowotny R. Instrumentation for dosimetry. Diagnostic Radiology Physics. A Handbook for Teachers and Students. Vienna, Austria: International Atomic Energy Agency; 2014

38. XCOM: Photon Cross Sections Database, NIST Standard Reference Database 8 (XGAM) National Institute of Standards and Technology, U.S. Department of Commerce, Gaithersburg, MD 20899. Available at: https://www.nist.gov/pml/xcom-photoncross-sections-database. Accessed 11 March 2019.

39. Micke A, Lewis DF, Yu X. Multichannel film dosimetry with nonuniformity correction. Med Phys. 2011;38:2523-2534.

\section{Reprint requests:}

Durer Iskanderani

Department of Oral and Maxillofacial Radiology

Faculty of Odontology

Malmö University

SE-205 06 Malmö

Sweden.

Durer.iskanderani@mau.se 



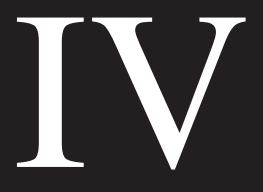





\title{
RESEARCH ARTICLE \\ Evaluation of a low-dose protocol for cone beam computed tomography of the temporomandibular joint
}

\author{
${ }^{1,2}$ Durer Iskanderani, ${ }^{1,3}$ Mats Nilsson, ${ }^{4}$ Per Alstergren, ${ }^{1,5,6}$ Xie-Qi Shi and ${ }^{1}$ Kristina Hellen-halme \\ ${ }^{1}$ Department of Oral and Maxillofacial Radiology, Faculty of Odontology, Malmö University, Malmö, Sweden; ${ }^{2}$ Department of \\ Oral and Maxillofacial Radiology, Faculty of Dentistry, King Abdulaziz University, Jeddah, Saudi Arabia; ${ }^{3}$ Department of Medical \\ Radiation Physics, Skåne University Hospital, Malmö, Sweden; ${ }^{4}$ Department of Orofacial Pain and Jaw Function, Faculty of \\ Odontology, Malmö University, Malmö, Sweden; ${ }^{5}$ Division of Oral Maxillofacial Radiology, Department of Clinical Dentistry, \\ Faculty of Medicine, University of Bergen, Bergen, Norway; ${ }^{6}$ Section of Oral Diagnostics and Surgery, Department of Dental \\ Medicine, Karolinska Institutet, Huddinge, Sweden
}

\begin{abstract}
Objective: Evaluation of cone beam CT (CBCT) examination with a low-dose scanning protocol for assessment of the temporomandibular joint (TMJ).

Methods: 34 adult patients referred for CBCT imaging of the TMJ underwent two examinations with two scanning protocols, a manufacturer-recommended protocol (default) and a lowdose protocol where the tube current was reduced to $20 \%$ of the default protocol. Three image stacks were reconstructed: default protocol, low-dose protocol, and processed (using a noise reduction algorithm) low-dose protocol. Four radiologists evaluated the images. The Sign test was used to evaluate visibility of TMJ anatomic structures and image quality. Receiver operating characteristic analyzes were performed to assess the diagnostic accuracy. $\kappa$ values were used to evaluate intraobserver agreement.

Results: With the low-dose and processed protocols, visibility of the TMJ anatomical structures and overall image quality were comparable to the default protocol. No significant differences in radiographic findings were found for the two low-dose protocols compared to the default protocol. The area under the curves $\left(\mathrm{A}_{z}\right)$ averaged for the low-dose and processed protocols, according to all observers, were 0.931 and 0.941 , respectively. Intraobserver agreement was good to very good.

Conclusion: For the CBCT unit used in this study, the low-dose CBCT protocol for TMJ examination was diagnostically comparable to the manufacturer-recommended protocol, but delivered a five times lower radiation dose. There is an urgent need to evaluate protocols for CBCT examinations of TMJ in order to optimize them for a radiation dose as low as diagnostically acceptable (the as low as diagnostically acceptable principle recommended by NCRP). Dentomaxillofacial Radiology (2020) 49, 20190495. doi: 10.1259/dmfr.20190495
\end{abstract}

Cite this article as: Iskanderani $\mathrm{D}$, Nilsson M, Alstergren P, Shi X-Q, Hellen-halme K. Evaluation of a low-dose protocol for cone beam computed tomography of the temporomandibular joint. Dentomaxillofac Radiol 2020; 49: 20190495.

Keywords: Cone-beam CT; Radiation dosage; TMJ

\section{Introduction}

In recent decades, cone beam computed tomography (CBCT) has become an essential examination tool in oral and maxillofacial radiology. CBCT produces multiplanar images of high spatial resolution, which for

Correspondence to: Durer Iskanderani, E-mail: durer.iskanderani@mau.se Received 04 December 2019; revised 28 March 2020; accepted 01 April 2020 many diagnostic tasks give information that is unattainable with two-dimensional projection radiography. In the diagnosis and treatment planning of patients with temporomandibular disorders (TMD), CBCT plays an effective role in assisting the clinicians when performing bony changes assessments with high diagnostic accuracy. ${ }^{1}$ In comparison to medical $\mathrm{CT}$, no significant 
difference in diagnostic accuracy was found between the two techniques; moreover, it is a cost- and dose-effective alternative.,3 Hence, it is considered as an appropriate imaging technique for evaluating bony changes of the temporomandibular joint (TMJ). ${ }^{4}$ However, the growing use of $\mathrm{CBCT}$, partly because of its availability and easiness of use, demands a critical evaluation of the relatively high patient radiation dose. Many different scanning protocols are available, and sometimes the urge from clinicians to have more or less noise free images can increase the radiation dose to patients without adding more information to the specific aim of the investigation. Thus, with the many CBCT scanners on the market today, clinicians together with medical physicists should ensure that the scanner they are using is being set for the lowest possible radiation dose, yet capable of providing acceptable image quality for the particular diagnostic task. 5

In current practice, most clinicians use the manufacturer-recommended exposure settings for the scanner in all clinical applications. This practice usually produces acceptable image quality, but might raise questions concerning the associated radiation dose. Attempts to optimize the patient radiation dose without sacrificing diagnostic outcome include approaches, such as optimizing tube current $(\mathrm{mA})$, tube voltage $(\mathrm{kV})$, and voxel size, selecting field of view (FOV) suitable to the diagnostic task, and using $180^{\circ}$ or $360^{\circ}$ of rotation. ${ }^{6-11}$

One practical approach of dose optimization is to adjust the scanner's tube current to a level that the dentist may obtain enough diagnostic information of a given task at the expense of acceptable loss of image quality. ${ }^{11,12}$ The structures in the TMJs have the advantage of appearing in relatively high contrast on CBCT scans, and are thus less susceptible to a reduction in exposure than for low-contrast structures. The possibility to establish if a degenerative disease is present or not may be easier to establish, in comparison to a thin periodontal ligament for endodontic questions. Radiographic images of the TMJ do not have to be very sharp with a high signal to noise ratio. Moreover, advanced denoising algorithms may further improve the image quality of scans made using a low-dose protocol.

There is clearly a need for a broader understanding of reduced-dose protocols related to subjective image quality in specific dental CBCT applications. Such protocols have already been assessed for some dental situations but not in the context of TMJ imaging in vivo. The aim of this study was thus to evaluate a low-dose protocol with and without image processing in $\mathrm{CBCT}$ examination of the TMJ.

Our hypothesis was that the low-dose protocol differed significantly compared to the default, manufacturerrecommended protocol in regard to the radiographic diagnostics on TMJ.

\section{Methods and materials}

The regional ethics review board in Lund, Sweden, approved this study (Dnr 2017/434). The study enrolled 34 adult patients (5 males and 29 females with a mean age of 57 years, ranged from 20 to 74 years) who had been referred for CBCT examination of the TMJ from the Department of Orofacial Pain and Jaw Function at Malmö University, Malmö, Sweden. For a power of 0.8 with a significant level of 0.05 , around 60 TMJs were required. The indication to perform a CBCT investigation of TMJ was related to the clinicians' questions after thorough history and clinical investigation. The main reason for the CBCT investigation was when the performed treatment failed. All participants underwent two consecutive CBCT examinations with different exposure settings. All study participants signed informed-consent forms. This form included information about the additional radiation dose, $20 \%$ extra, which is equivalent to less than one week of background exposure in Sweden.

\section{Cone beam CT examination}

Two CBCT examinations (Veraviewepocs 3D F40, J. Morita Corp., Kyoto, Japan) were performed both TMJs of each patient which resulted in a total number of $68 \mathrm{CBCT}$ volumes. The applied exposure protocols were: the default, manufacturer-recommended protocol (exposure settings: $90 \mathrm{kV}, 5 \mathrm{~mA}$, and $9.4 \mathrm{~s}$ ) and a lowdose protocol ( $90 \mathrm{kV}, 1 \mathrm{~mA}$ and $9.4 \mathrm{~s})$. All other parameters were kept constant throughout the examinations, i.e. $\mathrm{FOV}$ of $40 \times 40 \mathrm{~mm}, 180^{\circ}$ rotation, and head position with the Frankfurt plane paralleled and the midsagittal plane perpendicular in relation to the floor of the mouth. Patients were informed to sit still during exposure. Multiplanar data were reconstructed with a pixel size of $0.125 \mathrm{~mm}, 1 \mathrm{~mm}$ slice thickness, and $1 \mathrm{~mm}$ slice interval.

\section{Image evaluation}

68 reconstructed sagittal volumes were saved in DICOM format. These volumes were grouped into three stacks for display and processing using Image $\mathbf{J}$ (National Institutes of Health, Bethesda, MD): a default protocol stack, a low-dose protocol stack, and a processed low-dose stack with noise reduction (Figure 1). The processed stacks were created using an advanced noise reduction algorithm. ${ }^{13}$

We anonymized, randomly coded, and stored 204 data sets using Microsoft Excel Worksheet (Office Professional Plus 2016, Microsoft Corporation, Redmond, WA). Four (three senior and one junior) oral and maxillofacial radiologists evaluated all images. Before the study began, observer calibration was done on CBCT sagittal volumes of the first 25 stacks with a consensus on the instructions for assessing the images. The observers were blinded to any clinical or radiographic information and independently assessed the 


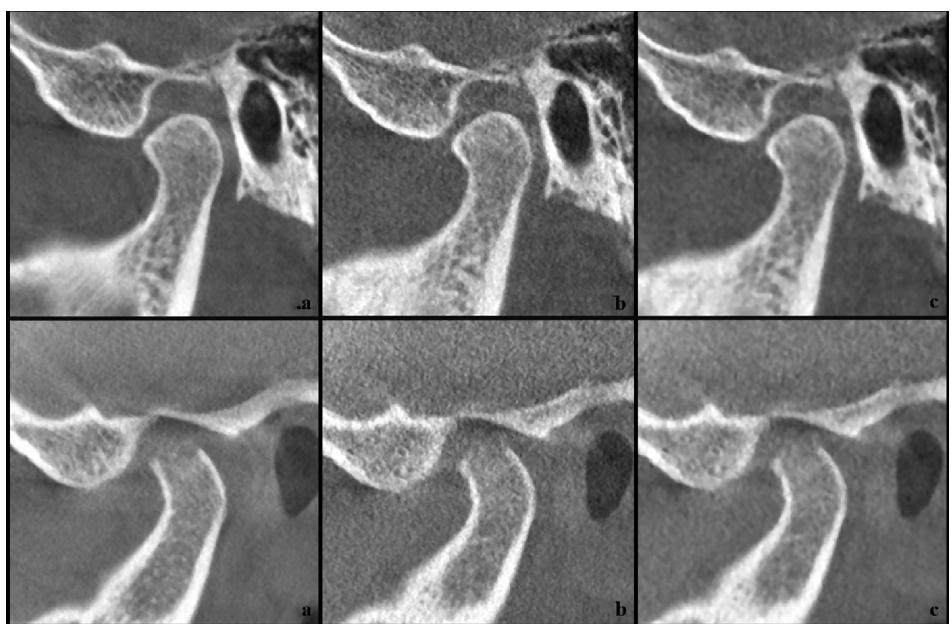

Figure 1 Examples of cone beam CT images for two temporomandibular joints using the three protocols (a) default, manufacturer recommended, (b) low-dose and (c) processed low-dose. $1(\mathrm{a}, \mathrm{b}, \mathrm{c})$ : case with no signs of degenerative joint disease; 2 (a, b, c) case with signs of degenerative joint disease.

visibility of five anatomical structures: the outlines of the condyle, the articular eminence, and the articular fossa and the trabecular patterns of the condyle and the temporal bone. All observers used the same type of viewing monitor, (Barco MDCC-6430, 8500 Kortrijk, Belgium) under the same viewing conditions with no time limitations. The observers were allowed to adjust brightness and contrast when necessary. A 3-point scale was applied to assess the visibility of each anatomical structure: 1 = definitely visible, 2 = questionably visible, and $3=$ not visible. Furthermore, the observers were asked to an overall impression on subjective image quality ranked as: $1=$ diagnostically acceptable, $2=$ diagnostically questionable, and $3=$ not diagnostically acceptable. To assess the radiographic finding, the observers were asked to record their level of confidence about the presence of degenerative joint disease (DJD) and were stated as $1=$ definitely not, $2=$ probably not, 3 = questionable, $4=$ probablyand $5=$ definitely according to Diagnostic Criteria of TMD (DC/TMD). ${ }^{14}$ Intraobserver agreement was determined by asking each observer to re-evaluate $40 \mathrm{TMJ}$ at an interval of at least 14 days and under the same conditions.

\section{Statistical analysis}

The five anatomical structures were pooled together in all settings for each observer and compared pairwise. For instance, the amount of "definitely visible" in the default protocol in relation to the number of "definitely visible" in the low-dose protocol. The overall image quality was evaluated in the same way. The results were analyzed using the Sign test ${ }^{15}$ with the level of significance at 0.05 .

The default protocol of the CBCT unit was considered to be the reference standard to establish if DJD was present or not, by two observers (XS, KHH). A consensus was reached in cases of disagreement. Receiver operating characteristic (ROC) curves were used to analyze the radiographic findings concerning the presence of DJD for all the observers. ${ }^{16}$ The area under the curves $\left(A_{z}\right)$ were calculated. Intraobserver agreement was estimated using $\kappa(\kappa)$ statistics. ${ }^{17}$ The values were interpreted according to the guidelines of Landis and Koch ${ }^{18}$ adapted by Altman. ${ }^{15}$

\section{Results}

Visibility of the five anatomical structures and overall image quality, according to all observers, showed no difference $(p=0.00)$, for the two low-dose protocols compared to the default protocol. Only one observer found improvement in processing the images for both structures' visibility and image quality between lowdose protocol and processed protocol, respectively ( $p=$ 0.79 (visibility), $p=1.00$ (image quality)).

The evaluation of the default protocol for the 68 TMJ cases showed that half of them were sound while the rest had DJD with varying osseous changes. When establishing the reference standard, the two observers disagreed in one of the cases, which had minor erosions on the superior surface of the condyle. 


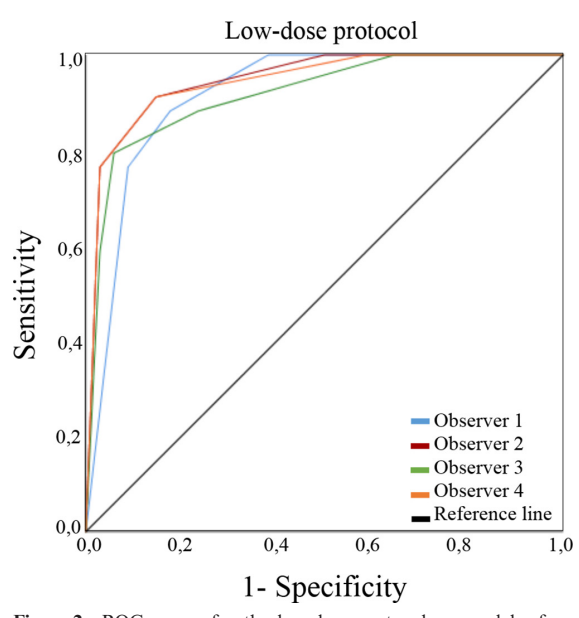

Figure 2 ROC curves for the low-dose protocol assessed by four observers according to the radiographic finding of degenerative joint disease. ROC, receiver operating characteristic.

ROC curves for the low-dose and processed protocols assessed by all the observers according to DJD presence are presented in Figures 2 and 3, respectively. Table 1 presents the different $A_{z}$ for each observer. The differences in the radiographic findings for the two

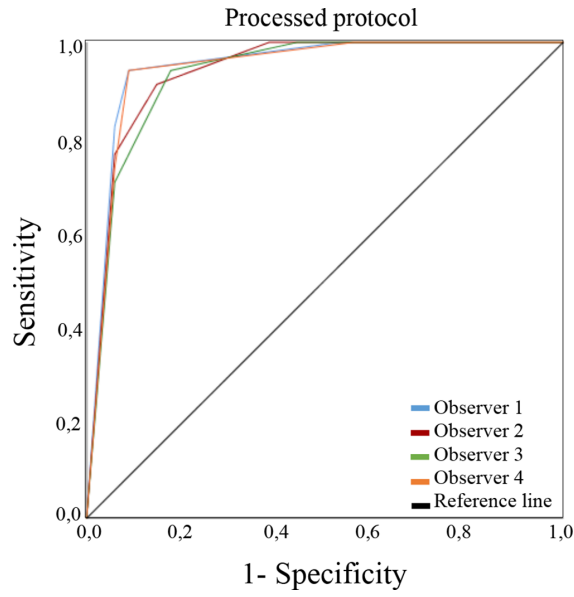

Figure 3 ROC curves for the processed protocol assessed by four Figure 3 ROC curves for the processed protocol assessed by four
observers according to the radiographic finding of degenerative joint disease. ROC, receiver operating characteristic low-dose protocols compared to the default one, as well as between them, were not significant. The average $\mathrm{A}$ for the observers were 0.931 for low-dose protocol and 0.941 for the processed protocol. Intraobserver agreement among the radiographic finding were good to very good for all observers $(\kappa$ values $=0.75,0.80,0.80,0.85$ ).

\section{Discussion}

Several studies in the literature have demonstrated that $\mathrm{CBCT}$ imaging exposed with low-dose protocol may provide diagnostically acceptable image quality for various dental indications..$^{6-11}$ The present study assessed a low-dose CBCT protocol for TMJ imaging, where tube current was lowered to just $20 \%$ of the manufacturer-recommended level in a clinical setting. In this study, the ethics review board approved that the potential benefit to future clinical practice for CBCT examination of the TMJ overcomes the increased radiation burden (equivalent to less than 1 week of background exposure in Sweden) for the patients included in the study. Therefore, we used an ethically approved study design that has been tested with different radiographic examinations. ${ }^{19-22}$

It is well known that manufacturer-recommended exposure settings for the CBCT scanners designed for dentistry vary widely, which results in varying amounts of radiation doses. Tube current $(\mathrm{mA})$ and exposure time (s) control the number of X-ray photons emitted; thus, higher $\mathrm{mAs}$ increases the measured signal from the sensor and decreases image noise. The $\mathrm{mA}$ setting is linearly proportional to radiation dose. ${ }^{23}$ Hence, when tube voltage and exposure time are constant, lowering the $\mathrm{mA}$ setting by $50 \%$ reduces the delivered dose by half and consequently the quantum noise in the resultant image will be higher. Pauwels et $\mathrm{a}^{12}$ reported minimal loss of image quality when reducing tube current compared to when reducing tube voltage. Another study by Sur $\mathrm{J}$ et al, ${ }^{11}$ confirmed the potential to reduce dose through a reduction in tube current.

In line with the recommendations ${ }^{24,25}$ for the use of any radiographic investigation, optimization should be done whenever possible in order to expose the patient to the lowest radiation dose according to the As Low As Diagnostically Acceptable (ALADA) principle, which is proposed as a variation of the acronym ALARA (as low as reasonably achievable) to emphasize the importance of optimization according to a given diagnostic task in medical imaging. ${ }^{25}$ High image quality in the sense of being noise free and having high resolution is not necessary in all clinical situations. Structural changes in the few anatomical structures that comprise the region of the TMJ are quite visible at exposure levels well below those giving the highest image quality. Usually, we try to find osseous changes when any form of DJD is suspected; thus, lower radiation dose protocols are possible. A high resolution might be needed for assessing fine pathological changes, however. Thus, the diagnostic 
Table 1 Area under the receiver operating characteristic curves $\left(\mathrm{A}_{2}\right)$ for assessing the presence of the degenerative joint disease, according to four observers as evaluated on cone-beam computed tomography images produced by three protocols: the low-dose (1 mA tube current) protocol, the processed (low-dose with noise reduction) protocol, and the reference standard ("default" manufacturer-recommended, $5 \mathrm{~mA}$ tube current) protocol

\begin{tabular}{lcccccc}
\hline & \multicolumn{3}{c}{ Low-dose protocol } & & \multicolumn{2}{c}{ Processed protocol } \\
\hline & $\mathrm{Az}$ & Standard error & $p$-value & $\mathrm{Az}$ & Standard error & $p$-value \\
Observer 1 & 0.918 & 0.036 & 0.000 & 0.949 & 0.029 & 0.000 \\
Observer 2 & 0.947 & 0.026 & 0.000 & 0.939 & 0.030 & 0.000 \\
Observer 3 & 0.917 & 0.034 & 0.000 & 0.933 & 0.032 & 0.000 \\
Observer 4 & 0.943 & 0.028 & 0.000 & 0.944 & 0.030 & 0.000 \\
\hline
\end{tabular}

task determines the level of image quality needed and, in turn, the degree of exposure parameter optimization. In our faculty, the demand for CBCT investigation of TMJ has decreased over time. Only patients where no explanation of their pain and no effect of treatment for TMD were found, and for those where other questions that possibly could be answered would be subject to a CBCT investigation. Thus, the CBCT examination of the TMJ were considered justified.

Our results demonstrated the ability of a low-dose protocol to image the TMJ sufficiently well without significant difference in diagnostic accuracy. Thus, this protocol is preferable, since the radiation dose was $20 \%$ of the dose delivered using the default protocol Kadesjö et $\mathrm{al}^{26}$ reported a $50 \%$ potential for dose reduction compared with the manufacturer's recommendation for the CBCT examination of TMJ. Comparable results were found for reduced dose protocols with other diagnostic tasks. ${ }^{6}, 10,11$

Reduction in dose is associated with degradation of image quality, principally due to the increased noise level. Noise influences both contrast resolution and spatial resolution and consequently, a representation of an object. However, in a sense of visual perception, the radiologist may still be able to see the object details and maintain diagnostic performance. When the diagnostic performance is influenced by a high level of noise, a noise reduction algorithm may be used to improve the image quality. Such programs filter out noise to varying degrees while preserving texture, contour, and fine details. ${ }^{13}$ The denoising method that we used was reported to be the technique of choice when very fine details are not required. ${ }^{27}$ We found that processing the images did not significantly improve the diagnostic accuracy (Table 1).

We used the default settings of the CBCT as a reference standard, although no "truth" can be obtained in this kind of clinical studies. We calculated the $\mathrm{A}$ in order to determine the validity of the two reduced-dose protocols for detecting the presence of DJD. Our findings revealed that both low-dose and processed protocols showed comparable diagnostic accuracy in relation to the reference standard (average $\mathrm{A}_{\mathrm{z}}=0.931$ and 0.941 , respectively), implying that the observers performance on detection of possible degenerative changes were comparable. A retrospective practice-based study found a similar result for pre-surgical implant assessment. ${ }^{9}$

It should be noted that these results were obtained in a real clinical setting, including factors such as motion artifacts that affect image quality. However, the limitations of this study could be that the results cannot be generalized to all CBCT machines. The number of observers chosen could be another limitation. However, the interobserver agreement was good, and therefore, further observers would, in our opinion, probably not contribute to any significant difference. A previous study by Hintze et $\mathrm{al}^{28}$ reported that the statistical power is dependent on the total number of evaluations, including the number of observers and surfaces evaluated for carious lesions, not on each separately. However, this kind of evaluation is known to show lower interobserver agreement due to the fact that these lesions are low contrast lesions. In comparison, TMJ structures appear with relatively high contrast.

As previous studies on low-dose protocols have also found adequate diagnostic image quality, our results could be considered together with these; it should be concluded that dose reduction achieved using a tube current below the recommendations of the manufacturer should always be investigated, in order to improve compliance with the ALADA principle. ${ }^{25}$ More important, a low-dose protocol should be the self-evident choice for younger patients, as the developing tissues of young patients are more radiosensitive and thus at a higher risk from X-ray exposure than adult tissues. ${ }^{29}$

\section{Conclusion}

The hypothesis that low-dose protocol would affect the radiographic diagnostics on TMJ could be rejected, which means that for the CBCT unit used in this study, the lowdose CBCT protocol for TMJ examination was diagnostically comparable to the manufacturer-recommended protocol but delivered a five times lower radiation dose. There is an urgent need to evaluate protocols for CBCT examinations of TMJ in order to optimize them for a radiation dose as low as diagnostically acceptable (the ALADA principle recommended by NCRP). 


\section{Acknowledgment}

The authors acknowledge Karin Näsström and Xin Feng for their participation in image observation. This study was financially supported by King Abdulaziz University.

\section{Funding}

This study was financially supported by King Abdulaziz University.

\section{REFERENCES}

1. Ma R-H, Yin S, Li G, Ma Ruo-han, Yin Shuang LG. The detection accuracy of cone beam CT for osseous defects of the temporomandibular joint: a systematic review and meta-analysis. Sci Rep 2016; 6: 34714. doi: https://doi.org/10.1038/srep34714

2. Honda K, Larheim TA, Maruhashi K, Matsumoto K, Iwai K. Osseous abnormalities of the mandibular condyle: diagnostic reliability of cone beam computed tomography compared with helical computed tomography based on an autopsy material. Dentomaxillofac Radiol 2006; 35: 152-7. doi: https://doi.org/10. $1259 / \mathrm{dmfr} / 15831361$

3. Sirin Y, Guven K, Horasan S, Sencan S. Diagnostic accuracy of cone beam computed tomography and conventional multislice spiral tomography in sheep mandibular condyle fractures. Dentomaxillofac Radiol 2010; 39: 336-42. doi: https://doi.org/10.1259/ $\mathrm{dmfr} / 29930707$

4. Tsiklakis K, Syriopoulos K, Stamatakis HC. Radiographic examination of the temporomandibular joint using cone beam computed tomography. Dentomaxillofac Radiol 2004; 33: 196 201. doi: https://doi.org/10.1259/dmfr/27403192

5. Lofthag-Hansen S, Thilander-Klang A, Gröndahl K. Evaluation of subjective image quality in relation to diagnostic task for cone beam computed tomography with different fields of view. Eur $J$ Radiol 2011; 80: 483-8. doi: https://doi.org/10.1016/j.ejrad.2010. 09.018

6. Liljeholm R, Kadesjö N, Benchimol D, Hellén-Halme K, Shi X-Q. Cone-Beam computed tomography with ultra-low dose protocols for pre-implant radiographic assessment: an in vitro study. Eur $J$ Oral Implantol 2017; 10: 351-9.

7. Hidalgo Rivas JA, Horner K, Thiruvenkatachari B, Davies J, Theodorakou C. Development of a low-dose protocol for cone beam CT examinations of the anterior maxilla in children. $\mathrm{Br}$ J Radiol 2015; 88: 20150559.. doi: https://doi.org/10.1259/bjr. 20150559

8. Pauwels R, Seynaeve L, Henriques JCG, de Oliveira-Santos C, Souza PC, Westphalen FH, et al. Optimization of dental CBCT exposures through $\mathrm{mAs}$ reduction. Dentomaxillofac Radiol 2015; 44: 20150108. doi: https://doi.org/10.1259/dmfr.20150108

9. Dawood A, Brown J, Sauret-Jackson V, Purkayastha S. Optimization of cone beam CT exposure for pre-surgical evaluation of the implant site. Dentomaxillofac Radiol 2012; 41: 70-4. doi: https:// doi.org/10.1259/dmfr/16421849

10. Neves FS, Souza TdeC, de-Azevedo-Vaz SL, Campos PSF, Bóscolo FN. Influence of cone-beam computed tomography milliamperage settings on image quality of the mandibular third molar region. Oral Radiol 2014; 30: 27-31. doi: https://doi.org/10. 1007/s11282-013-0132-6

11. Sur J, Seki K, Koizumi H, Nakajima K, Okano T. Effects of tube current on cone-beam computerized tomography image quality for presurgical implant planning in vitro Oral Surg Oral Med Oral Pathol Oral Radiol Endod 2010; 110: e29-33. doi: https://doi.org/ 10.1016/j.tripleo.2010.03.041

\section{Human and animal rights statement:}

All procedures followed were in accordance with the ethical standards of the responsible committee on human experimentation (institutional and national) and with the Helsinki Declaration of 1964 and later versions. Informed consent was obtained from all patients for being included in the study.

12. Pauwels R, Silkosessak O, Jacobs R, Bogaerts R, Bosmans H, Panmekiate $\mathrm{S}$. A pragmatic approach to determine the optimal $\mathrm{kVp}$ in cone beam CT: balancing contrast-to-noise ratio and radiation dose. Dentomaxillofac Radiol 2014; 43: 20140059. doi: https://doi.org/10.1259/dmfr.20140059

13. Rudin LI, Osher S, Fatemi E. Nonlinear total variation based noise removal algorithms. Physica D 1992; 60(1-4): 259-68. doi: https://doi.org/10.1016/0167-2789(92)90242-F

14. Ahmad M, Hollender L, Anderson Q, Kartha K, Ohrbach R, Truelove EL, et al. Research diagnostic criteria for temporomandibular disorders (RDC/TMD): development of image analysis criteria and examiner reliability for image analysis. Oral Surg Oral Med Oral Pathol Oral Radiol Endod 2009; 107: 844-60. doi: https://doi.org/10.1016/j.tripleo.2009.02.023

15. Altman DG. Practical statistics for medical research (1st edn. London, UK: Chapman and Hall; 1991.

16. Metz CE. Basic principles of ROC analysis. Semin Nucl Med 1978; 8: 283-98. doi: https://doi.org/10.1016/S0001-2998(78)80014-2

17. Cohen J. A coefficient of agreement for nominal scales. Educ Psychol Meas 1960; 20: 37-46. doi: https://doi.org/10.1177/ 001316446002000104

18. Landis JR, Koch GG. The measurement of observer agreement for categorical data. Biometrics 1977; 33: 159-74 doi: https://doi. org/10.2307/2529310

19. Phexell E, Åkesson A, Söderberg M, Bolejko A. Intra- and interrater reliability in a comparative study of cross-sectional and spiral computed tomography pelvimetry methods. Acta Radiol Open 2019; 8: 205846011985518. doi: https://doi.org/10.1177/ 2058460119855187

20. Meyer E, Labani A, Schaeffer M, Jeung M-Y, Ludes C, Meyer A et al. Wide-volume versus helical acquisition in unenhanced chest CT: prospective Intra-patient comparison of diagnostic accuracy and radiation dose in an ultra-low-dose setting. Eur Radiol 2019; 29: 6858-66, doi: https://doi.org/10.1007/s00330 019-06278-6

21. Lee NK, Kim S, Hong SB, Kim TU, Ryu H, Lee JW, et al. LowDose CT with the adaptive statistical iterative reconstruction $\mathrm{V}$ technique in abdominal organ injury: comparison with routinedose CT with filtered back projection. AJR Am J Roentgenol 2019; 213: 659-66. doi: https://doi.org/10.2214/AJR.18.20827

22. Pan S, Su JJ, Syed J, Moore C, Israel G, Singh D. Reduced dose computed tomography: the effects of voltage reduction on density measurements of urolithiasis. J Endourol 2019; 33: 682-6. doi: https://doi.org/10.1089/end.2019.0149

23. Qu X-min, Li G, Ludlow JB, Zhang Z-yan, Ma X-chen, Qu X Zhang Z, Ma X. Effective radiation dose of ProMax 3D conebeam computerized tomography scanner with different dental protocols. Oral Surg Oral Med Oral Pathol Oral Radiol Endod 2010; 110: 770-6. doi: https://doi.org/10.1016/j.tripleo.2010.06. 013 
24. The I. The 2007 recommendations of the International Commission on radiological protection. ICRP publication 103. Ann ICRP 2007; 37: 1-332. doi: https://doi.org/10.1016/j.icrp.2007.10.003

25. Bushberg JT. Science, radiation protection, and the NCRP building on the past, looking to the future. In: Fiftieth Annual Meeting Program of NCRP: Achievements of the Past 50 Years and Addressing the Needs of the Future ; : Bethesda, MD; 2014. pp. 5. -7 .

26. Kadesjö N, Benchimol D, Falahat B, Näsström K, Shi X-Q. Evaluation of the effective dose of cone beam CT and multislice CT for temporomandibular joint examinations at optimized exposure levels. Dentomaxillofac Radiol 2015; 44: 20150041. doi: https:/ doi.org/10.1259/dmfr.20150041
27. Shahmoradi M, Lashgari M, Rabbani H, Qin J, Swain M A comparative study of new and current methods for dental micro-CT image denoising. Dentomaxillofac Radiol 2016; 45 20150302. doi: https://doi.org/10.1259/dmfr.20150302

28. Hintze $\mathrm{H}$, Frydenberg M, Wenzel A. Influence of number of surfaces and observers on statistical power in a multiobserver ROC radiographic caries detection study. Caries Res 2003; 37: 200-5. doi: https://doi.org/10.1159/000070445

29. Demeter S, Applegate KE, Perez M. Internet-Based ICRP resource for healthcare providers on the risks and benefits of medical imaging that uses ionising radiation. Ann ICRP 2016; 45(1 Suppl): 148-55. doi: https://doi.org/10.1177/ 0146645316637786 


ISBN 978-9I-7877-I06-6 (print)

ISBN 978-9I-7877-IO7-3 (pdf)

MALMÖ UNIVERSITY 20506 MALMÖ, SWEDEN WWW.MAU.SE 